\title{
THE THEORY OF THE HYDROGEN MOLECULE ION, SCALAR BEAMS, AND SCATTERING BY SPHEROIDS
}

by

Rufus M Boyack

\author{
A thesis \\ submitted to the Victoria University of Wellington \\ in fulfilment of the \\ requirements for the degree of \\ Master of Science \\ in Physics. \\ Victoria University of Wellington \\ 2011
}




\section{Abstract}

Schrödinger's equation for the hydrogen molecule ion and the Helmholtz equation are separable in prolate and oblate spheroidal coordinates respectively. They share the same form of the angular equation. The first task in deriving the ground state energy of the hydrogen molecule ion, and in obtaining finite solutions of the Helmholtz equation, is to obtain the

physically allowed values of the separation of variables parameter. The separation parameter is not known analytically, and since it can only have certain values, it is an important parameter to quantify. Chapter 2 of this thesis investigates an exact method of obtaining the separation parameter. By showing that the angular equation is solvable in terms of confluent Heun functions, a new method to obtain the separation parameter was obtained. We showed that the physically allowed values of the separation of variables parameter are given by the zeros of the Wronskian of two linearly dependent solutions to the angular equation. Since the Heun functions are implemented in Maple, this new method allows the separation parameter to be calculated to unlimited precision.

As Schrödinger's equation for the hydrogen molecule ion is related to Helmholtz's equation, this warranted investigation of scalar beams. Tightly focused optical and quantum particle beams are described by exact solutions of the Helmholtz equation. In Chapter 3 of this thesis we investigate the applicability of the separable spheroidal solutions of the scalar Helmholtz equation as physical beam solutions. By requiring a scalar beam solution to satisfy certain physical constraints, we showed that the oblate spheroidal wave functions can only represent nonparaxial scalar beams when the angular function is odd, in terms of the angular variable. This condition ensures the convergence of integrals of physical quantities over a cross-section of the beam and allows for the physically necessary discontinuity in phase at $z=0$ on the ellipsoidal surfaces of otherwise constant phase. However, these solutions were shown to have a discontinuous longitudinal derivative.

Finally, we investigated the scattering of scalar waves by oblate and prolate spheroids whose symmetry axis is coincident with the direction of the incident plane wave. We developed a phase shift formulation of scattering 
by oblate and prolate spheroids, in parallel with the partial wave theory of scattering by spherical obstacles. The crucial step was application of a finite Legendre transform to the Helmholtz equation in spheroidal coordinates. Analytical results were readily obtained for scattering of Schrödinger particle waves by impenetrable spheroids and for scattering of sound waves by acoustically soft spheroids. The advantage of this theory is that it enables all that can be done for scattering by spherical obstacles to be carried over to the scattering by spheroids, provided the radial eigenfunctions are known. 


\section{Acknowledgments}

I would like to express my gratitude to my supervisor, Professor John Lekner, who has made my Masters a very enjoyable one. It has been a pleasure to learn, not only theoretical physics, but also physics research, from a physicist, and person, I admire a lot. The work in this thesis has been the combined efforts of John and I, and without his guidance and expertise would not be what it is. I am grateful for all his help and support. I would also like to thank Victoria University of Wellington for funding through the Masters by Thesis Scholarship, which has made this research possible. Finally, to my Mother and Uncle Calvin Breakenridge, thank you both for providing many helpful comments on this manuscript, I am indebted to you both for all your help and support. 
Whatever Nature has in store for mankind, unpleasant as it may be, men must accept, for ignorance is never better than knowledge.

Enrico Fermi (1901-1954) 


\section{Contents}

1 Introduction 1

1.1 The hydrogen molecule ion . . . . . . . . . . . . . . . 1

1.2 Scalar beams . . . . . . . . . . . . . . . . . . . 3

1.3 Scattering of scalar waves by spheroids . . . . . . . 5

2 The hydrogen molecule ion $\quad 7$

2.1 Separation of electron and nuclear terms . . . . . . . . 7

2.2 The electronic wave equation . . . . . . . . . . . . . 11

2.3 The angular equation . . . . . . . . . . . . . . 16

2.4 The Wronskians . . . . . . . . . . . . . . . . . . . . . . . 19

2.4.1 The angular Wronskian . . . . . . . . . . . . . . . 19

2.4 .2 The radial Wronskian . . . . . . . . . . . . . . 24

2.5 The radial equation . . . . . . . . . . . . . 26

2.6 The ground state of the hydrogen molecule ion . . . . . . . 30

2.7 Anti-bonding excited state . . . . . . . . . . . . . 35

2.8 Non-zero orbital angular momentum _ . . . . . . . . . . 40

2.9 An invariant of the motion . . . . . . . . . . . . . . . 42

3 Scalar and Electromagnetic beams 46

3.1 Introduction . . . . . . . . . . . . . . 47

3.2 Oblate spheroidal coordinates . . . . . . . . . . . 50

3.3 Angular and radial equations . . . . . . . . . . . . 53

3.4 Asymptotics of the radial solutions . . . . . . . . . . 55

3.5 Integral relations for confluent Heun functions . . . . . . . . 61

3.6 Nonparaxial and paraxial scalar beams . . . . . . . . . 67 
3.6.1 Confluent Heun beams . . . . . . . . . . . . . . 67

3.6 .2 Oblate spheroidal beams . . . . . . . . . . . . 78

3.6.3 Non-existence of separable spheroidal beams . . . . . . 91

3.7 Oblate spheroidal electromagnetic beams . . . . . . . . . . 94

3.8 Bessel beams and the Bateman solution . . . . . . . . . . . 98

3.8.1 Generalized Bessel beams . . . . . . . . . . . . . 98

3.8.2 Bateman solution of the wave equation . . . . . . . 103

4 Scattering of scalar waves by spheroids 106

4.1 Introduction . . . . . . . . . . . . . . . . . 107

4.2 Wave equation for oblate spheroidal scatterers . . . . . . . 110

4.3 Scattering by oblate spheroids . . . . . . . . . . . . . 114

4.4 Long wave scattering by oblate spheroids . . . . . . . . . 117

4.5 Scattering by prolate spheroids . . . . . . . . . . . 122

5 Discussion and Conclusions 126

A The Heun equation 130

A.1 General Heun equation . . . . . . . . . . . . . . 130

A.2 Confluent Heun equation . . . . . . . . . . . . . 133

A.3 Double confluent Heun equation . . . . . . . . . . . . 135

A.4 Biconfluent Heun equation . . . . . . . . . . . . . . 136

A.5 Triconfluent Heun equation . . . . . . . . . . . . 136

$\begin{array}{lll}\text { B Abstracts of papers } & 137\end{array}$ 


\section{Chapter 1}

\section{Introduction}

This thesis investigates three problems, which are related through the separability of the Schrödinger equation in prolate spheroidal coordinates, and the separability of the Helmholtz equation in oblate spheroidal coordinates. The Schrödinger equation for the hydrogen molecule ion is separable in prolate spheroidal coordinates, and leads to an angular and radial equation. Separation of the Helmholtz equation in oblate spheroidal coordinates also leads to an angular and radial equation. The remarkable result is that the angular equation for the hydrogen molecule ion is the same as the angular equation for the Helmholtz equation. Initially the hydrogen molecule ion was studied, but because of the relation between the Schrödinger equation and the Helmholtz equation, this warranted investigation of free space scalar beams, and also the theory of scattering of scalar waves by spheroids. An introduction for each individual topic is presented in the following sections.

\section{$1.1 \quad$ The hydrogen molecule ion}

The simplest of all molecules is the hydrogen molecule ion, consisting of two protons and one electron. The study of this molecule is important both theoretically, and also to provide an understanding of the nature of electron bonding. It is the only quantum mechanical three body problem which is separable, in the Born-Oppenheimer approximation, due to the fact that there is no electron-electron repulsion. 
The solution of this problem would lead to an exact (possibly transcendental) relationship between the energy of the electron $(E)$ and the internuclear separation of the two protons $(R)$. However, by performing separation of variables, a separation parameter $\mu(\lambda)$ is introduced, where $\lambda=-E R^{2} / 2$, which can only have certain physical values. An analytic expression for the separation parameter is not yet known.

The original study of the hydrogen molecule ion was performed by Pauli, and Niessen $[1,2]$. In both these treatments the old, semi-classical, quantum theory was used. This led to results which were in disagreement with experiment. Following the development of the Schrödinger equation, Burrau [3] was able to obtain the ground state energy of the hydrogen molecule ion. Burrau showed that the Schrödinger equation is separable in elliptic (prolate spheroidal) coordinates. This led to two equations linked through the separation parameter $\mu(\lambda)$. The method of Burrau was to convert the two linear second-order differential equations into first-order non-linear Riccati differential equations. Solving the two equations numerically, Burrau was able to obtain the ground state energy in agreement with experiment [9].

More sophisticated treatments were given following Burrau's initial work. Wilson [4, 5] solved the angular equation using a series solution, which gave a series representation of the separation parameter $\mu(\lambda)$ in terms of $\lambda$. Wilson then considered the radial equation and, by utilizing an asymptotic expansion, obtained an infinite continued fraction relating $R, \mu(\lambda)$, and $\lambda$. However, Jaffé [6] showed that this asymptotic solution did not lead to the correct infinite continued fraction. Building upon Wilson's work, Jaffé [6] solved the radial equation using a series solution which led to the correct infinite continued fraction relating $R, \mu(\lambda)$, and $\lambda$. The infinite continued fraction, along with the series representation for $\mu(\lambda)$, then allowed the ground state energy of the hydrogen molecule ion to be determined.

Hylleraas [7] solved the angular equation in terms of an expansion of associated Legendre functions. By imposing the requirements of a convergent series solution, Hylleraas obtained a determinantal equation which must be solved to give the physical values of the separation parameter. This method also led to the correct series representation of the separation parameter. 
Hylleraas then considered certain expansions of Laguerre polynomials in order to solve the radial equation. This led to another determinantal equation which, when combined with the angular result, gave the electron energy as a function of the internuclear distance.

There are also many treatments of the hydrogen molecule ion based on variational theory or perturbation theory. For a discussion of the treatments using variational theory see Chapter 12, section 42 of [8]. Perturbative methods are given in [9].

The lack of an analytic representation of the separation parameter was the initial motivation for this work. The major goal of this research was to:

- Derive an exact relation between the separation parameter $\mu(\lambda)$ and the parameter $\lambda$.

We shall give a method of solution, based on the zeros of the Wronskian of the angular functions, which gives the separation parameter exactly. The method of our solution for $\mu(\lambda)$ will be compared with those outlined previously, and we will investigate the applicability of this method to solve the radial equation. As will be shown in subsequent Chapters, the separation parameter is important for not only the hydrogen molecule ion, but many other applications, such as scalar beams, and scattering of scalar waves by spheroids.

\subsection{Scalar beams}

The advancements in producing tightly focused laser and particle beams has raised the necessity to have exact representations of such beams. In the case of laser beams, where the diameter of the beam can be of the order of a few wavelengths, the optical fields must be described by nonparaxial beams. Such tightly focused beams, in both the optical and quantum particle case, can only be described by exact solutions of the scalar Helmholtz equation.

Optical beams which are weakly focused can be described by the paraxial Gaussian beam. In the focal region of a tightly focused beam, however, the Gaussian beam is not an adequate representation of a physical beam. 
The description of optical beams beyond the paraxial approximation, developed by Lax et al. [10], is based on introducing correction terms into the paraxial solution which represent nonparaxiality. The complete behaviour of a physical beam solution must, however, be described by an exact solution of the scalar Helmholtz equation.

Exact solutions of the scalar Helmholtz equation, based on complexsource and sink points, have been proposed as physical beams. Deschamps [11] introduced an outgoing spherical wave, which has a source point located at a complex position, as a possible scalar beam. The beam constructed was found to have properties of the paraxial Gaussian beam and remains an exact solution of the Helmholtz equation. A fundamental flaw of this beam is that it has an inherent singularity on the circle $\rho=b$ in the focal plane, where $b$ is the length of the focal region of the beam, and consequently the beam solution diverges on this circle.

In order to overcome this divergence Sheppard and Saghafi $[12,13,14]$ replaced the complex source outgoing spherical wave by a non-singular superposition of an incoming and outgoing spherical wave. This beam has been expressed in oblate spheroidal coordinates and Ulanowski and Ludlow [15] generalized it to a set of exact beam solutions known as spherical Bessel beams. These beams, however, are also not physical. The construction of a beam by a superposition of counter-propagating wavefronts does not represent true free space propagation. Furthermore, the energy required to produce subsets of such beams has been found to be infinite [16].

Another set of possible beam solutions has recently been suggested by utilizing the fact that the Helmholtz equation is separable in oblate spheroidal coordinates. Rodríguez-Morales and Chávez-Cerda [17] have suggested that physical free space beams may be represented by the oblate spheroidal wave functions. 
It is at this point where the research that we planned to undertake comes in. The major goals for this part of research were the following:

- Derive exact solutions of the Helmholtz equation in oblate spheroidal coordinates.

- Investigate the asymptotic nature of these solutions and ensure they represent physical beams.

- Derive exact free space electromagnetic beams from the scalar beam solutions of the Helmholtz equation.

Our study of the solutions proposed in [17] as physical beams resulted in showing that only subsets of the oblate spheroidal wave functions may represent physical scalar beams [18]. However, this subset of beams also has nonphysical aspects, which shall be discussed in both the scalar and electromagnetic cases.

\subsection{Scattering of scalar waves by spheroids}

The scalar Helmholtz equation is separable in both the oblate and prolate spheroidal coordinate systems. Most previous research on scattering by spheroidal shaped obstacles has relied on this fact, with calculations largely using the spheroidal wave functions, which Chapter 3 of this thesis discusses with respect to free space scalar beams. It is this application of the possible scalar beam solutions, namely spheroidal wave functions, to scattering by spheroids, which provided the stimulus to study this problem.

Initial research on scattering by spheroids was performed by Rayleigh [19], who investigated the acoustically soft scattering by a disk, which is the limiting form of an oblate spheroid. By relating the electrical capacitance of the disk to the scattered wave, Rayleigh was able to derive the scattering amplitude, in the long-wavelength limit, by solving a potential problem instead. 
The scattering of sound by prolate spheroids has also been a problem under thorough investigation. The solution of plane wave scattering by a rigid prolate spheroid (acoustically hard) has been given by Spence and Granger in terms of the spheroidal wave functions [20]. The case of acoustically soft prolate spheroidal scattering has also been investigated by Senior [21].

Long-wavelength scattering by both acoustically soft and acoustically hard spheroidal bodies has been considered by Senior [22], with an extension to scattering by a dielectric body [23]. Senior shows that, for acoustically soft objects, the scattered field may be expanded in a series of increasing powers of $k$, where $k$ is the wavenumber. The leading term of this series is of the order of $O\left(k^{0}\right)$, and proportional to the electrostatic capacity, in analogy with Rayleigh's work. In the case of acoustically hard bodies, Senior has related the scattered wave, whose leading term is proportional to $k^{2}$, to the magnetic polarizability tensor of the same geometry.

Short-wavelength scattering by rigid prolate spheroids has been investigated by Lauchle [24], who shows that, approximately, in this limit the total-cross section is proportional to twice the geometrical area of the spheroid. Sammelmann et al. [25] have also investigated the short-wavelength scattering by rigid prolate spheroids, while Ye [26] has considered lowfrequency acoustic scattering by gas filled prolate spheroids in liquids.

The scattering by spheroids has in general been in relation to acoustic waves. The case of soft acoustic scattering by a spheroid is equivalent to hard quantum-mechanical scattering by an impenetrable spheroid. It is this relation, and also the relation between the Helmholtz equation and the free space Schrödinger equation, which motivated this research.

Instead of utilizing the separability of the Helmholtz equation we utilized a finite Legendre transform. This enabled the theory of scattering by spheroids to be put in a form in one-to-one correspondence with the phase shift formulation of scattering by spherical scatterers developed by Rayleigh, see section 334 of [27]. 


\section{Chapter 2}

\section{The hydrogen molecule ion}

This Chapter investigates the ground state and certain excited states of the hydrogen molecule ion. Utilizing the Born-Oppenheimer approximation we shall show that the Schrödinger equation for the electron is separable in prolate spheroidal coordinates. For both the angular and radial equations we shall derive solutions in terms of HeunC functions. Following this, we shall give a new method of obtaining the separation parameter exactly. The physical values of the separation of variables parameter shall be shown to be given by the zeros of the Wronskian of two linearly dependent angular solutions. The method is applied to certain virtual excited states of the hydrogen molecule ion, and comparison with the results of other methods is made. A discussion is also presented of the possible extension of this method to the radial equation. Finally, we shall discuss an invariant of the motion, and show that the separable eigenvalue equation for the invariant leads to the same separable equations as the Schrödinger equation.

\subsection{Separation of electron and nuclear terms}

Before discussing the hydrogen molecule ion itself, a brief account of the Born-Oppenheimer [28] approximation is given, since this principle is in essence the foundation of molecular quantum mechanics. 
The solution of Schrödinger's wave equation for all except the simplest molecules is a very difficult problem. However, the empirical results of molecular spectroscopy indicate that the energy values of a molecule can be conveniently separated into several parts, namely the electronic energy, the vibrational energy of the nuclei, and the rotational energy of the molecule. This suggests it is possible to devise a method where, initially, the energy of the electrons is determined for various arrangements of the nuclei fixed in space. The stable state will then correspond to one in which the combined electronic and internuclear energy is a minimum. The nuclei will then undergo vibrations about their equilibrium positions, and the molecule as a whole will rotate about its centre of mass.

Born and Oppenheimer were able to show, by a power series expansion in terms of the ratio of the electron and nuclei masses, that an approximate solution to the wave equation is obtainable by such a method. The method consists of first solving the wave equation for the electrons alone, with the nuclei fixed, as a function of the nuclei coordinates. Following this, a wave equation for the nuclei is solved, where the energy of the electrons acts as the potential function. The physical premise behind this idea is that the mass of the nuclei are much larger than the mass of the electrons, thus the electrons undergo many cycles of their motion before the nuclear configuration changes, allowing one to essentially fix the nuclei coordinates.

The complete wave equation for a molecule consisting of $r$ nuclei and $s$ electrons is

$$
\sum_{j=1}^{r} \frac{1}{M_{j}} \nabla_{j}^{2} \psi+\frac{1}{m_{e}} \sum_{i=1}^{s} \nabla_{i}^{2} \psi+\frac{2}{\hbar^{2}}(E-V) \psi=0,
$$

in which $m_{e}$ is the electron mass, $M_{j}$ is the mass of the $j$ th nucleus, $\nabla_{j}^{2}$ is the Laplace operator in terms of the coordinates of the $j$ th nucleus, and $\nabla_{i}^{2}$ is the Laplacian for the $i$ th electron. $E$ is the energy eigenvalue of the molecule, and $V$ is the potential energy function of the system given by

$$
V=\sum_{i, i^{\prime}} \frac{e^{2}}{r_{i i^{\prime}}}+\sum_{j, j^{\prime}} \frac{Z_{j} Z_{j^{\prime}} e^{2}}{r_{j j^{\prime}}}-\sum_{i, j} \frac{Z_{j} e^{2}}{r_{i j}}
$$

with the interpretation of the sums being over each pair of particles once. $Z_{j}$ is the atomic number of the $j$ th nucleus. 
Let $y$ represent the $3 r$ coordinates of the $r$ nuclei, relative to axes fixed in space, and $x$ represent the $3 s$ coordinates of the $s$ electrons. Let $\alpha$ represent the quantum numbers associated with the motion of the electrons and $\beta$ denote the quantum numbers associated with the motion of the nuclei. The result of Born and Oppenheimer is that an approximate solution for $\Psi_{\alpha, \beta}(x, y)$ can be obtained in the form

$$
\Psi_{\alpha, \beta}(x, y)=\psi_{\alpha}(x, y) \psi_{\alpha, \beta}(y) .
$$

The functions $\psi_{\alpha}(x, y)$ are called the electronic wave functions, and correspond to the eigenfunctions of the electronic term in the Hamiltonian, relative to fixed nuclear separation. They do not depend on the nuclear quantum numbers, but are functions of the nuclear separation. The wave equation to be solved in order to obtain the electronic wave functions is given by

$$
\sum_{i=1}^{s} \nabla_{i}^{2} \psi_{\alpha}(x, y)+\frac{2 m_{e}}{\hbar^{2}}\left[U_{\alpha}(y)-V(x, y)\right] \psi_{\alpha}(x, y)=0 .
$$

The potential function $V(x, y)$ is the complete potential function of Eqn. 2.2. The function $U_{\alpha}(y)$ represents the characteristic electronic energy and is dependent on the nuclear separation. Once we have obtained the characteristic electronic energy $U_{\alpha}(y)$ as a function of the nuclei coordinates, for a given set of values of the electronic quantum numbers $\alpha$, we next obtain expressions for the nuclear wave functions.

The results of Born and Oppenheimer show that the nuclear wave functions are the acceptable solutions of a nuclear wave equation, in which the potential is given by the electronic energy terms. The nuclear wave equation thus has the form

$$
\sum_{j=1}^{r} \frac{1}{M_{j}} \nabla_{j}^{2} \psi_{\alpha, \beta}(y)+\frac{2}{\hbar^{2}}\left[E_{\alpha, \beta}-U_{\alpha}(y)\right] \psi_{\alpha, \beta}(y)=0 .
$$

$E_{\alpha, \beta}$ are the characteristic energy values of the entire molecule, and are dependent on the electronic quantum numbers $\alpha$, and the nuclear quantum numbers $\beta$. 
In summary, the general treatment of solving Schrödinger's equation for a molecule is given by:

- Separating out the nuclear and electronic dependent terms in the wave function.

- Solving a wave equation for the electrons with the nuclei fixed to obtain the electronic energy.

- Solving a wave equation for the nuclei using the electronic energy as the potential.

In some cases it might not be possible to separate the nuclear and electronic motions and one must consider coupling between the two. There are also other approximations, such as the diabatic approximation, which take into account nuclear kinetic energy terms (non-separable terms in the molecular Hamiltonian) and allow a better representation of the state of a molecule. By comparison with experimental results, the applicability of the BornOppenheimer approximation in the case of the hydrogen molecule ion shall be justified.

In the zeroth approximation the energy levels of a molecule are independent of spin. By incorporating relativistic considerations the $(2 S+1)$ degenerate energy levels of a molecule are split, and consequently depend on the projection of the spin on the nuclear axis. This spin-axis interaction comprises of spin-orbit, spin-spin, and spin-rotation interactions. The classification of molecular levels with non-zero spin depends on the relative magnitudes of the spin-axis interaction and the rotation of the molecule.

For the $\Sigma$ terms of a molecule (to be defined in the next section) it is found that the rotational energy levels of the molecule, which arise from a fine splitting of the vibrational energy levels of the molecule, predominate over multiplet splitting due to spin effects. In section 2.6 we shall give the energy of the hydrogen molecule ion due to only the electron and internuclear energy, and nuclear vibrational energy. The analysis of multiplet splitting due to spin, and rotation of the molecule, may be found in Chapter 11 of [29]. 


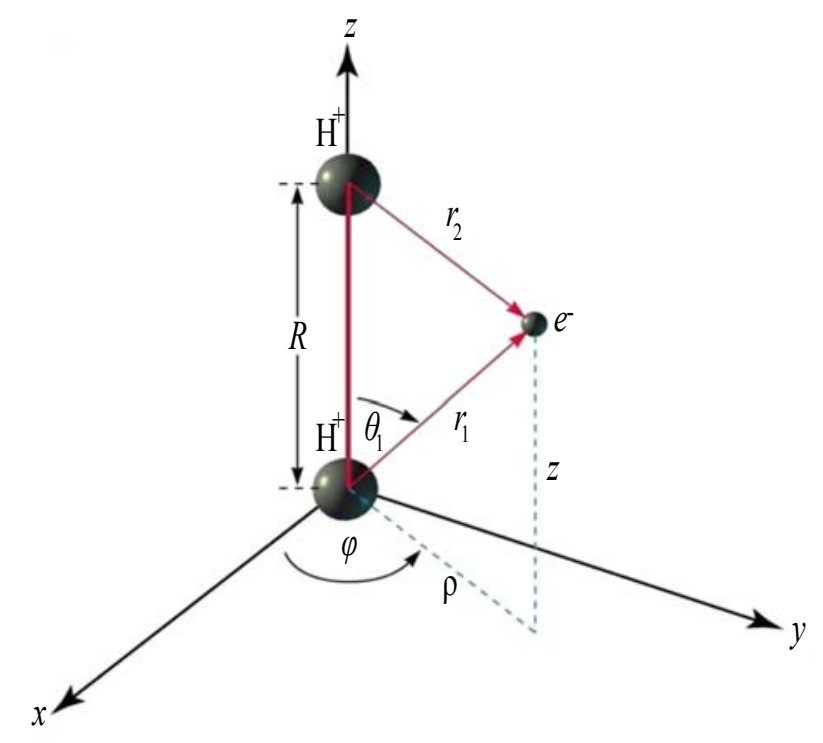

Figure 2.1: The geometry of $\mathrm{H}_{2}^{+}$with two protons separated a distance $R$, and an electron at a distance $r_{1}$ from proton 1 and $r_{2}$ from proton 2 .

\subsection{The electronic wave equation}

The first step in solving Schrödinger's equation for the hydrogen molecule ion $\left(\mathrm{H}_{2}^{+}\right)$, shown in Fig. 2.1, is to solve for the electronic wave functions and the characteristic electron energies as functions of the nuclear separation distance $R$. The electronic Hamiltonian for $\mathrm{H}_{2}^{+}$is given by

$$
H_{e}=-\frac{\hbar^{2}}{2 m_{e}} \nabla^{2}-\frac{e^{2}}{r_{1}}-\frac{e^{2}}{r_{2}} .
$$

A natural coordinate system to study $\mathrm{H}_{2}^{+}$is bipolar coordinates, whose geometry depends on $r_{1}, r_{2}$, and $\phi$ which are shown in Fig. 2.1. In bipolar coordinates the distances $r_{1}$ and $r_{2}$ become

$$
\begin{aligned}
& r_{1}^{2}=\rho^{2}+z^{2}, \\
& r_{2}^{2}=\rho^{2}+(R-z)^{2} .
\end{aligned}
$$

In cylindrical polar coordinates the Laplacian is given by

$$
\nabla^{2}=\partial_{\rho}^{2}+\frac{1}{\rho} \partial_{\rho}+\partial_{z}^{2}+\frac{1}{\rho^{2}} \partial_{\phi}^{2}
$$


which on conversion to bipolar coordinates becomes

$$
\nabla^{2}=\partial_{r_{1}}^{2}+\frac{2}{r_{1}} \partial_{r_{1}}+\partial_{r_{2}}^{2}+\frac{2}{r_{2}} \partial_{r_{2}}+\frac{r_{1}^{2}+r_{2}^{2}-R^{2}}{r_{1} r_{2}} \partial_{r_{1}} \partial_{r_{2}}+\frac{1}{\rho^{2}} \partial_{\phi}^{2},
$$

where

$$
\rho^{2}=-\frac{\left[\left(r_{1}+r_{2}\right)^{2}-R^{2}\right]\left[\left(r_{1}-r_{2}\right)^{2}-R^{2}\right]}{4 R^{2}} .
$$

In order to simplify the notation atomic units [au] shall be used, in which the units of length and energy are given by $\hbar^{2} / m_{e} e^{2}$ (Bohr radius) and $m_{e} e^{4} / \hbar^{2}$ (one half this unit equals a Rydberg). Thus, Schrödinger's equation for the electron in the field of the two nuclei is given by the expression

$$
\begin{gathered}
\left\{-\frac{1}{2}\left[\partial_{r_{1}}^{2}+\frac{2}{r_{1}} \partial_{r_{1}}+\partial_{r_{2}}^{2}+\frac{2}{r_{2}} \partial_{r_{2}}+\frac{r_{1}^{2}+r_{2}^{2}-R^{2}}{r_{1} r_{2}} \partial_{r_{1}} \partial_{r_{2}}\right]-\frac{1}{r_{1}}-\frac{1}{r_{2}}\right. \\
\left.+\frac{2 R^{2}}{\left[\left(r_{1}+r_{2}\right)^{2}-R^{2}\right]\left[\left(r_{1}-r_{2}\right)^{2}-R^{2}\right]} \partial_{\phi}^{2}\right\} \psi\left(r_{1}, r_{2}, \phi\right)=E \psi\left(r_{1}, r_{2}, \phi\right) .
\end{gathered}
$$

The electron energy $(E)$ must then have the energy due to the repulsion of the nuclei added to it in order to obtain the total energy. This results in a positive shift of the electron energy giving

$$
E_{\text {total }}(R)=E+\frac{1}{R} \text {. }
$$

Bipolar coordinates are not useful in the sense that Schrödinger's equation is still non-separable in its current form. However, in 1927 Burrau [3] was able to show that the electronic wave equation separates in elliptic coordinates. Following Burrau we introduce the elliptic coordinates $\xi$ and $\eta$ defined as:

$$
\begin{aligned}
\xi & =\frac{r_{1}+r_{2}}{R}, \\
\eta & =\frac{r_{1}-r_{2}}{R} .
\end{aligned}
$$

The surfaces $\xi=$ constant are confocal ellipsoids of revolution, with the nuclei at the foci, whereas the surfaces $\eta=$ constant are confocal hyperboloids. On the introduction of these coordinates, with $\rho^{2}=(R / 2)^{2}\left(\xi^{2}-1\right)\left(1-\eta^{2}\right)$ and $z=(R / 2)(\xi \eta+1)$, the Laplacian becomes

$$
\begin{array}{r}
\nabla^{2}=\frac{4}{R^{2}\left(\xi^{2}-\eta^{2}\right)}\left\{\partial_{\xi}\left(\xi^{2}-1\right) \partial_{\xi}+\partial_{\eta}\left(1-\eta^{2}\right) \partial_{\eta}\right. \\
\left.+\left[\frac{1}{\xi^{2}-1}+\frac{1}{1-\eta^{2}}\right] \partial_{\phi}^{2}\right\} .
\end{array}
$$


Schrödinger's time-independent equation for the $\mathrm{H}_{2}^{+}$energy eigenstates is thus given by

$$
\begin{array}{r}
\left\{\partial_{\xi}\left(\xi^{2}-1\right) \partial_{\xi}+\partial_{\eta}\left(1-\eta^{2}\right) \partial_{\eta}+2 R \xi+\frac{R^{2}}{2} E\left(\xi^{2}-\eta^{2}\right)\right. \\
\left.+\left[\frac{1}{\xi^{2}-1}+\frac{1}{1-\eta^{2}}\right] \partial_{\phi}^{2}\right\} \psi(\xi, \eta, \phi)=0
\end{array}
$$

By defining $\psi$ as the product function

$$
\psi(\xi, \eta, \phi)=X(\xi) Y(\eta) \Phi(\phi)
$$

the following three linear second-order ordinary differential equations, each in terms of a single variable, are obtained

$$
\begin{aligned}
\frac{d^{2} \Phi}{d \phi^{2}}+m^{2} \Phi & =0, \\
\frac{d}{d \eta}\left\{\left(1-\eta^{2}\right) \frac{d Y}{d \eta}\right\}+\left(\lambda \eta^{2}-\frac{m^{2}}{1-\eta^{2}}-\mu\right) Y & =0, \\
\frac{d}{d \xi}\left\{\left(\xi^{2}-1\right) \frac{d X}{d \xi}\right\}+\left(-\lambda \xi^{2}+2 R \xi-\frac{m^{2}}{\xi^{2}-1}+\mu\right) X & =0,
\end{aligned}
$$

where $\lambda=-E R^{2} / 2$ is non-negative, since $E<0$ for bound states, and $\mu$ is the separation of variables parameter. The range of the variable $\xi$ is from 1 to $\infty$, and of $\eta$ from -1 to 1 . The equations are linked through the parameter $\lambda$ and separation parameter $\mu$. In order to obtain the energy eigenvalues the relation between these two parameters must be determined.

The angular differential equation possesses regular singular points at $\eta=$ \pm 1 . The physical values of $\mu$ are obtained by having a solution $Y(\eta)$ which is finite at both end points. This leads to only certain $\mu_{m n}(\lambda)(m, n=0,1,2, \ldots)$ which give a physical solution [30, 31].

The familiar $\phi$ equation can be readily solved, and is found to have physical solutions only for discrete $m$ :

$$
\Phi(\phi)=\exp ( \pm i m \phi), \quad m=0,1,2, \ldots
$$


In this context, since the two protons do not produce a centrally symmetric field, angular momentum is not itself conserved, but the projection of the angular momentum onto the nuclear axis is conserved. Physically the integer $m$ corresponds to the angular momentum along the nuclear axis. It is an experimentally known fact that the ground state of a molecule is usually one in which $m=0$, so we henceforth set $m=0$ to obtain the ground state. (There are certain exceptions to this rule, for instance, the ground state of the molecule NO is an $m=1^{2} \Pi$ term.)

Since the ground state of $\mathrm{H}_{2}^{+}$is symmetric in $r_{1}$ and $r_{2}$ we require a solution even in $\eta$. Therefore, this limits the acceptable $\mu_{m n}(\lambda)$ separation parameters to only the subset $\mu_{m n}(\lambda), m=0, n=0,2,4, \ldots$ The separation parameter for the ground state of $\mathrm{H}_{2}^{+}$is thus $\mu_{00}(\lambda)$.

In the study of molecular physics the projection of the angular momentum onto the nuclear axis is usually denoted by the symbol $\Lambda$. The states $\Lambda=0,1$, 2 are given the symbols $\Sigma, \Pi, \Delta, \ldots$ etc. Thus the state $m=0$ corresponds to a $\Sigma$ state. Reflection of the molecule in a plane passing through the nuclear axis does not affect the molecule's energy, but the value of $\Lambda$ is reversed in sign, consequently all except the $\Sigma$ states are doubly degenerate. That is, for each energy value, there corresponds two different states which differ in the projection of the orbital angular momentum along the nuclear axis. ${ }^{1}$ The $\Sigma$ states are not changed upon reflection and so are non-degenerate. The wave function of a $\Sigma$ state can thus only be multiplied by a constant as a result of the reflection. Double reflection is equivalent to the identity transformation, and so the eigenvalues of this reflection operator (for $\Sigma$ states) are either \pm 1 . The states which change sign, and those which do not change sign upon reflection, are denoted as $\Sigma^{-}$and $\Sigma^{+}$.

Another symmetry in the hydrogen molecule ion results from a change in sign of the electron coordinates (nuclei coordinates unchanged).

\footnotetext{
${ }^{1}$ It is actually observed, if one takes into account the interaction between the electron state and the rotation, along with higher effects, that the energy of a state with $\Lambda \neq 0$ is split into two levels close together. This phenomenon is called lambda doubling and can be accounted for through perturbation theory. The correct wave functions are then the sum and difference of those corresponding to $\Lambda$ and $-\Lambda$. The effect is usually only important for $\Pi$ terms.
} 
In general, for a molecule with two identical nuclei, this symmetry arises because there is a centre of symmetry at the point bisecting the line joining the nuclei. The Hamiltonian remains invariant to such a change and this transformation operator also commutes with the orbital angular momentum operator. This enables the states to be labelled depending on the parity of the wave function. For a given $\Lambda$, even (g - gerade) states are invariant to such a change, while odd ( $u$ - ungerade) states change sign.

Furthermore, in a diatomic molecule, the electron state of the molecule is characterized by the total spin $S$ of all the electrons in the molecule. If $S$ is non-zero then there is a degeneracy of $2 S+1$ with respect to the directions of the total spin (ignoring fine structure). The number $2 S+1$ is called the multiplicity, as is the case for atoms, and is written as an index before the greek letter indicating the projection of the angular momentum along the nuclear axis. For example, ${ }^{2} \Pi_{u}$ denotes an odd electron state with $S=1 / 2$ and $\Lambda=1$. This results in the complete description of the electron state given by ${ }^{2 S+1} \Lambda_{g, u}^{( \pm)}$with the $( \pm)$included only for $\Sigma$ states. The hydrogen molecule ion has only one electron, with spin $S=1 / 2$, and so the multiplicity in all cases is equal to 2 . The hydrogen molecule ion ground state experimentally is found to be ${ }^{2} \Sigma_{g}^{+}$, and it is this state which shall be primarily investigated.

In order to solve the angular part of the hydrogen molecule ion problem the relation between $\mu$ and $\lambda$ must be obtained. By solving the $Y(\eta)$ equation it will be found that there is a unique $\mu_{m n}(\lambda)$ which leads to a physical solution. Using this $\mu_{m n}(\lambda)$ the relation between $R$ and $\lambda$ can subsequently be found by solving the $X(\xi)$ equation. This will determine the electronic energy as a function of the nuclear separation and will complete the solution of the electronic wave equation.

The procedure followed by Hylleraas to solve for the $\mu_{m n}(\lambda)$ and $\lambda$ relation will be discussed next, followed by a new method which relies on the linear dependence of solutions to the angular equation. In subsequent sections the separation parameter $\mu$ will be denoted $\mu_{m n}(\lambda)$ to indicate it is the unique function which exhibits the correct $\lambda$ dependence. Similarly, $R(\lambda)$ shall denote the unique $R$ which exhibits the correct $\lambda$ dependence; an arbitrary $\mu$ shall be denoted as $\mu$, and an arbitrary $R$ shall be denoted by $R$ respectively. 


\subsection{The angular equation}

The differential equation given in Eqn. 2.20 is called an angular equation because the variable $\eta$ extends from -1 to +1 , which is equivalent to the range traversed by the argument $z=\cos (\theta)$ of the associated Legendre functions. Hylleraas [7] proposed a solution to the angular equation in terms of these functions given by

$$
Y(\eta)=\sum_{l=|m|}^{\infty} c_{l} P_{l}^{|m|}(\eta)
$$

in which the coefficients $c_{l}$ are independent of $\eta$. Substituting this expression into the angular equation, and by comparison with the differential equation satisfied by the associated Legendre functions, we obtain the equation

$$
\sum_{l=|m|}^{\infty} c_{l}\left[\lambda \eta^{2}-\mu-l(l+1)\right] P_{l}^{|m|}(\eta)=0
$$

The term $\eta^{2} P_{l}^{|m|}(\eta)$ can be expressed in terms of $P_{l-2}^{|m|}(\eta), P_{l}^{|m|}(\eta)$, and $P_{l+2}^{|m|}(\eta)$ by use of the recursion formula

$$
\begin{aligned}
\eta^{2} P_{l}^{|m|} & =\frac{(l+|m|)(l+|m|-1)}{(2 l-1)(2 l+1)} P_{l-2}^{|m|} \\
& +\left\{\frac{(l-|m|+1)(l+|m|+1)}{(2 l+1)(2 l+3)}+\frac{(l-|m|)(l+|m|)}{(2 l-1)(2 l+1)}\right\} P_{l}^{|m|} \\
& +\frac{(l-|m|+1)(l-|m|+2)}{(2 l+1)(2 l+3)} P_{l+2}^{|m|}
\end{aligned}
$$

which may be obtained on double application of the recurrence relation between $P_{l}^{|m|}(\eta), P_{l-1}^{|m|}(\eta)$, and $P_{l+1}^{|m|}(\eta)$, namely

$$
(2 l+1) \eta P_{l}^{|m|}(\eta)=(l+|m|) P_{l-1}^{|m|}(\eta)+(l-|m|+1) P_{l+1}^{|m|}(\eta) .
$$

The Legendre functions are an orthogonal set over the physical range of $\eta$. Therefore, in order for the sum to vanish for all $\eta$, their coefficients must all vanish. This gives the following recurrence relation between the coefficients: 


$$
\begin{aligned}
& \frac{(l-|m|-1)(l-|m|)}{(2 l-3)(2 l-1)} \lambda c_{l-2} \\
+ & {\left[\left\{\frac{(l-|m|+1)(l+|m|+1)}{(2 l+1)(2 l+3)}+\frac{(l-|m|)(l+|m|)}{(2 l-1)(2 l+1)}\right\} \lambda-\mu-l(l+1)\right] c_{l} } \\
+ & \frac{(l+|m|+2)(l+|m|+1)}{(2 l+3)(2 l+5)} \lambda c_{l+2}=0
\end{aligned}
$$

The recurrence relation obtained in Eqn. 2.27 can be viewed as an infinite system of simultaneous linear homogeneous equations in the unknown coefficients $c_{l}$ (with $l$ even or odd). In order that the system possess a nontrivial solution, the determinant of the matrix of coefficients must vanish. This determinantal equation will then give the relation between $\lambda$ and $\mu$.

The matrix of coefficients for even $l$ (starting from $l=0$, with $m=0$ ) is

$$
M=\left[\begin{array}{ccccc}
\frac{\lambda}{3}-\mu & \frac{2 \lambda}{15} & 0 & 0 & \ldots \\
\frac{2 \lambda}{3} & \frac{11 \lambda}{21}-\mu-6 & \frac{4 \lambda}{21} & 0 & \ldots \\
0 & \frac{12 \lambda}{35} & \frac{39 \lambda}{77}-\mu-20 & \frac{30 \lambda}{143} & \cdots \\
\vdots & \vdots & \vdots & \vdots & \ddots
\end{array}\right]
$$

The matrix in Eqn. 2.28 only has non-zero elements on the main diagonal and the adjacent diagonals. Such a matrix allows an LU decomposition into a product of a lower and an upper triangular matrix:

$$
L U=\left[\begin{array}{cccc}
1 & 0 & 0 & \ldots \\
l_{0} & 1 & \ldots & \ldots \\
\vdots & \vdots & \vdots & \ddots
\end{array}\right]\left[\begin{array}{cccc}
d_{0} & u_{0} & \ldots & \ldots \\
0 & d_{1} & u_{1} & \ldots \\
\vdots & \vdots & \vdots & \ddots
\end{array}\right]=\left[\begin{array}{cccc}
d_{0} & u_{0} & 0 & \ldots \\
l_{0} d_{0} & d_{1}+l_{0} u_{0} & u_{1} & \ldots \\
\vdots & \vdots & \vdots & \ddots
\end{array}\right]
$$

If we express the recurrence relation given in Eqn. 2.27 as

$$
\alpha(l) c_{l-2}+\beta(l) c_{l}+\gamma(l) c_{l+2}=0
$$

then the $\alpha, \beta$, and $\gamma$ coefficients can be identified by comparison with the matrix in Eqn. 2.28. 
For the even $l$ case we deduce that

$$
\begin{aligned}
d_{0} & =\frac{\lambda}{3}-\mu=\beta(0), \\
u_{0} & =\frac{2 \lambda}{15}=\gamma(0), \\
l_{0} d_{0} & =\frac{2 \lambda}{3}=\alpha(2) \text { etc. }
\end{aligned}
$$

Using the properties of determinants we find that

$$
\operatorname{det}(M)=\operatorname{det}(L) \operatorname{det}(U)=\operatorname{det}(U)=\Pi_{0}^{\infty} d_{n}=0,
$$

so only the elements $d_{n}$ are needed to solve the determinantal equation. The diagonal elements are determined by

$$
\begin{aligned}
& \qquad d_{n}=\beta(2 n)-\frac{\alpha(2 n) \gamma(2 n-2)}{d_{n-1}}, \\
& \text { starting with } d_{0}=\beta(0)=\frac{\lambda}{3}-\mu .
\end{aligned}
$$

The solution to the infinite determinant gives a transcendental relation between $\mu$ and $\lambda$. If we set $d_{N}=0$ in the product in Eqn. 2.34, then we obtain an equation for $\mu$ of the $(\mathrm{N}+1)$ th degree. The first two such approximations $(\mathrm{N}=0,1)$ give a linear and a quadratic equation for $\mu$. In the quadratic case the $\mu$ value obtained will correspond to $\mu_{00}(\lambda)$ and $\mu_{02}(\lambda)$, which have the following series representations as a function of $\lambda$

$$
\begin{aligned}
& \mu_{00}(\lambda)=\frac{1}{3} \lambda+\frac{2}{135} \lambda^{2}+\frac{4}{8505} \lambda^{3}+O\left(\lambda^{4}\right), \\
& \mu_{02}(\lambda)=-6+\frac{11}{21} \lambda+O\left(\lambda^{2}\right) .
\end{aligned}
$$

This successful method employed by Hylleraas allows successive approximations to determine $\mu$ as a function of $\lambda$. The convergence to the true function, $\mu_{m n}(\lambda)$, is rapid and this result represents a great achievement in understanding the eigenvalue relationship in the $\mathrm{H}_{2}^{+}$problem. This now allows the $\mu$ dependency in the radial equation to be eliminated, which on solving will give the desired relation between $R$ and $\lambda$, and ultimately determine the electronic energy as a function of the internuclear separation. The next section shall investigate an exact method that gives the $\mu_{m n}(\lambda)$ and $\lambda$ relation. 


\subsection{The Wronskians}

\subsubsection{The angular Wronskian}

The angular and radial equations are linear second-order homogeneous differential equations with three singular points. Both equations have two regular singular points and an irregular singular point at infinity. The Frobenius-Fuchs method of power series can be carried through giving solutions to each of the angular and radial equations which are non-singular at the two regular singular points. By Fuchs's theorem a Taylor series expansion of the solution to the angular equation, about the ordinary point $\eta=0$, is convergent out to the nearest singularity $\eta^{2}=1$, see Chapter 3 of [32].

First we consider the angular equation, which has been studied in great detail previously because of its relation to the spheroidal wave equation [31]. The solution to this has already been given in terms of a series of Legendre functions, which gives the series representation of $\mu_{m n}(\lambda)$, but here a new method to derive the $\mu_{m n}(\lambda)$ and $\lambda$ relation is developed. It is an exact, transcendental relation and does not require an infinite series representation.

The angular equation, as derived previously, is given by

$$
\frac{d}{d \eta}\left\{\left(1-\eta^{2}\right) \frac{d Y}{d \eta}\right\}+\left(\lambda \eta^{2}-\frac{m^{2}}{1-\eta^{2}}-\mu\right) Y=0 .
$$

We shall consider the $m=0$ case of Eqn. 2.39, since physically this corresponds to the ground state, i.e. $\mu_{m n}(\lambda)=\mu_{0 n}(\lambda), n=0,1,2,3, \ldots$. (For convenience of notation $\mu_{0 n}(\lambda), n=0,2,4, \ldots$ shall be denoted $\mu_{e}(\lambda)$, whereas $\mu_{0 n}(\lambda), n=1,3,5, \ldots$ shall be denoted $\mu_{o}(\lambda)$, which stand for even and odd respectively.)

If the substitution $x=1-\eta^{2}$ is made in the angular equation then the following differential equation for the function $f(x)=Y(\eta)$ is obtained

$$
x(x-1) f^{\prime \prime}+\left(\frac{3}{2} x-1\right) f^{\prime}+\frac{1}{4}(\lambda x+\mu-\lambda) f=0 .
$$

The prime denotes differentiation with respect to $x$. This differential equation is a non-symmetrical confluent Heun equation. 
A discussion of the Heun equation and its confluent forms will be presented in the appendix, but for the moment the solutions to the aforementioned equation will be investigated. (The Heun equation is a generalization of the hypergeometric differential equation, but it now contains 4 regular singularities. Eqn. 2.40 is one of its confluent forms.)

Eqn. 2.40 has two regular singular points at $x=0$ and $x=1$. Using the Frobenius-Fuchs power series method we obtain two power series solutions, each one non-singular at one of the corresponding regular singularities. The two even angular solutions to Eqn. 2.40, one regular at $x=1$ the other regular at $x=0$, are given by

$$
\begin{aligned}
& f_{1}(x)=\operatorname{HeunC}\left(0,-\frac{1}{2}, 0,-\frac{\lambda}{4}, \frac{\mu+1}{4}, 1-x\right), \\
& f_{0}(x)=\operatorname{HeunC}\left(0,0,-\frac{1}{2}, \frac{\lambda}{4}, \frac{\mu-\lambda+1}{4}, x\right),
\end{aligned}
$$

Performing the transformation back in terms of the variable $\eta$, the even solutions to the angular equation are

$$
\begin{aligned}
& Y_{0}^{\text {even }}(\eta)=\operatorname{HeunC}\left(0,-\frac{1}{2}, 0,-\frac{\lambda}{4}, \frac{\mu+1}{4}, \eta^{2}\right) \\
& Y_{1}^{\text {even }}(\eta)=\operatorname{HeunC}\left(0,0,-\frac{1}{2}, \frac{\lambda}{4}, \frac{\mu-\lambda+1}{4}, 1-\eta^{2}\right) .
\end{aligned}
$$

$Y_{0}^{\text {even }}(\eta)$ is regular at the ordinary point $\eta=0$, for any $\mu . Y_{1}^{\text {even }}(\eta)$ is regular at the regular singular points $\eta^{2}=1$, for any $\mu$. If, however, $\mu=\mu_{e}(\lambda)$ then both these solutions shall be non-singular at $\eta=0$ and $\eta^{2}=1$. At the correct $\mu_{e}(\lambda)$ the two solutions become linearly dependent, but are linearly independent when $\mu \neq \mu_{e}(\lambda)$, for a given lambda. Thus $\mu_{e}(\lambda)$ can be deduced from the property of linear dependence. This is expressed in the Wronskian.

Mathematically this method is represented in the concise form

$$
W_{A}^{e v e n}(\mu, \lambda, \eta)=Y_{0}^{e v e n}(\eta) Y_{1}^{e v e n}(\eta)^{\prime}-Y_{1}^{e v e n}(\eta) Y_{0}^{e v e n}(\eta)^{\prime}=0 \Leftrightarrow \mu=\mu_{e}(\lambda) .
$$

The prime denotes differentiation with respect to $\eta$. 
The Wronskian method is based on the following theorem due to Józef Maria Hoëne-Wroński [33]:

Theorem 1. If the functions $f_{1}, f_{2}, \ldots f_{n}$ have $n-1$ continuous derivatives on the interval $(-\infty, \infty)$, and if the Wronskian of these functions is not identically zero on $(-\infty, \infty)$, then these functions form a linearly independent set of vectors in $C^{(n-1)}(-\infty, \infty)$.

The Wronskian is not in general equal to zero only for linearly dependent solutions. This is because solving the Wronskian equal to zero gives a necessary, and not sufficient, condition for linear dependence. For example, if $f_{1}(x):=x^{2}, f_{2}(x):=\left\{f_{1}(x) x \geq 0,-f_{1}(x) x<0\right\}$, then these two solutions have zero Wronskian (for all $x$ ), but are linearly independent. However, since the solutions to the angular equation are analytic, and the Wronskian is zero for all $\eta$, at the correct $\mu_{m n}(\lambda)$, the Wronskian then gives a necessary and sufficient condition for linear dependence. The importance of the Wronskian is that the angular solutions are linearly dependent if and only if their Wronskian is equal to zero, therefore, by solving the Wronskian equal to zero we will obtain all the physical $\mu_{m n}(\lambda)$.

The Wronskian method can be summarized as follows:

- The solutions are linearly dependent when the correct separation parameter is used if and only if the Wronskian is equal to zero.

- Solving the Wronskian equal to zero will thus determine the separation parameter.

The even Wronskian for the two even angular confluent Heun solutions is given by

$$
\begin{array}{r}
W_{A}^{\text {even }}(\mu, \lambda, \eta)=-2 \eta\left\{\left[\operatorname{HeunC}\left(0,0,-\frac{1}{2}, \frac{\lambda}{4}, \frac{\mu-\lambda+1}{4}, 1-\eta^{2}\right)\right.\right. \\
\left.\times \operatorname{HeunCPrime}\left(0,-\frac{1}{2}, 0,-\frac{\lambda}{4}, \frac{\mu+1}{4}, \eta^{2}\right)\right]+\left[\operatorname{HeunC}\left(0,-\frac{1}{2}, 0,-\frac{\lambda}{4}, \frac{\mu+1}{4}, \eta^{2}\right)\right. \\
\left.\left.\times \operatorname{HeunCPrime}\left(0,0,-\frac{1}{2}, \frac{\lambda}{4}, \frac{\mu-\lambda+1}{4}, 1-\eta^{2}\right)\right]\right\},
\end{array}
$$

where HeunCPrime denotes the derivative of a confluent Heun function. The value of $\eta$ is arbitrary, and may be set to $1 / \sqrt{2}$, since this gives the value of $x$ which is between the two endpoints of Eqn. 2.41 and Eqn. 2.42. 


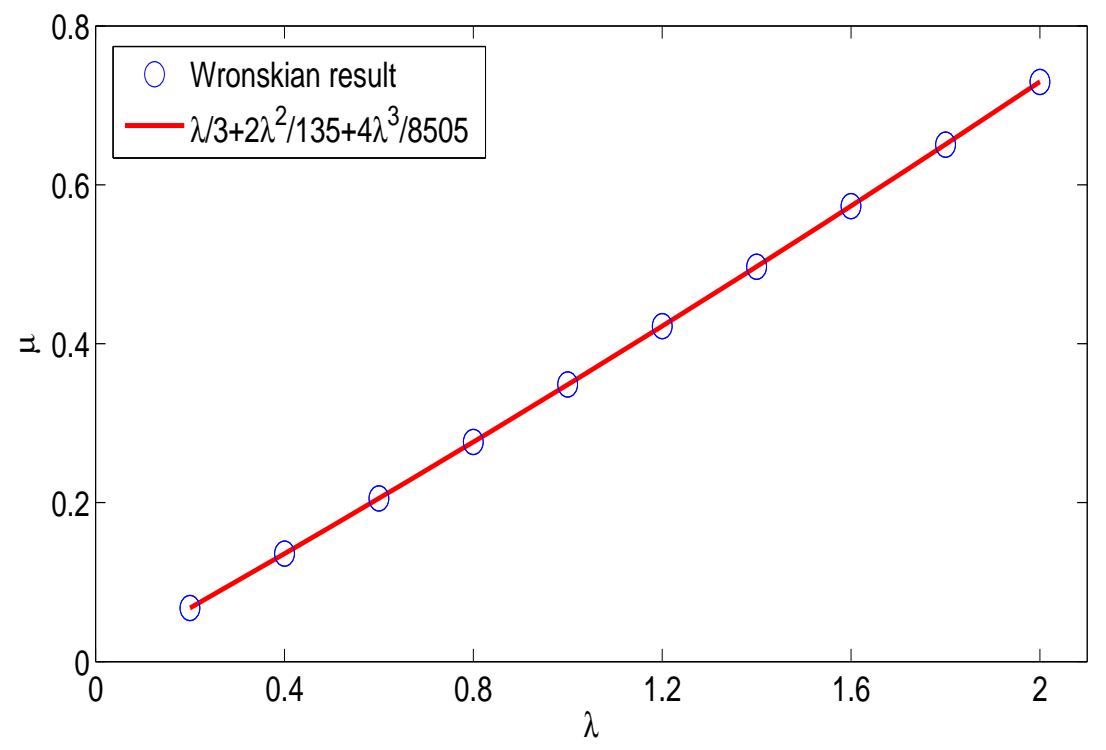

Figure 2.2: Comparison between the $\mu_{00}(\lambda)$ value computed using the Wronskian method and with the series method for a range of $\lambda$ values.

In Fig. 2.2 the results of solving Eqn. 2.45 for a set of $\lambda$ values is compared against the series result given in Eqn. 2.37. Fig. 2.2 shows complete agreement between the values of $\mu_{00}(\lambda)$ obtained by the Wronskian method, and by the series method. The values of $\lambda$ used here were small, but the method can be implemented for a $\lambda$ of any size. An advantage of the Wronskian is that by solving Eqn. 2.45 we obtain all possible $\mu_{0 n}(\lambda), n=0,2,4, \ldots$ Thus all the roots to the single expression in Eqn. 2.46 give all physically possible $\mu_{0 n}(\lambda), n=0,2,4, \ldots$

The exact formula for the Wronskian of the solutions to the angular differential equation may be derived by use of Abel's identity. It follows from the angular differential equation that the Wronskian has the form

$$
W_{A}^{e v e n}(\mu, \lambda, \eta)=\frac{F^{e v e n}(\mu, \lambda)}{1-\eta^{2}} .
$$

The Wronskian function, $F^{e v e n}(\mu, \lambda)$, is therefore zero at one of the physical values $\mu_{0 n}(\lambda), n=0,2,4, \ldots$ For $\lambda=1$, the even $\mu_{0 n}(\lambda)$ values are approximately $0.34860,-5.4868,-19.4953, \ldots$, which are equivalent with those in $[30,31]$. 


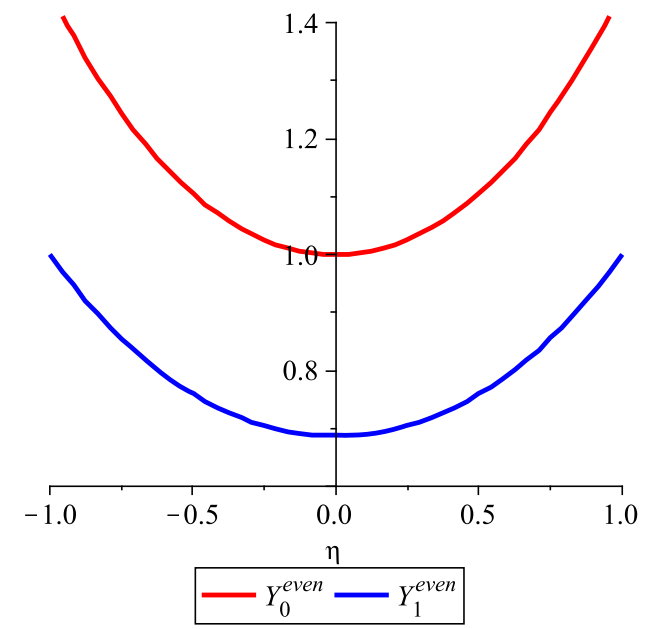

(a) Linearly dependent

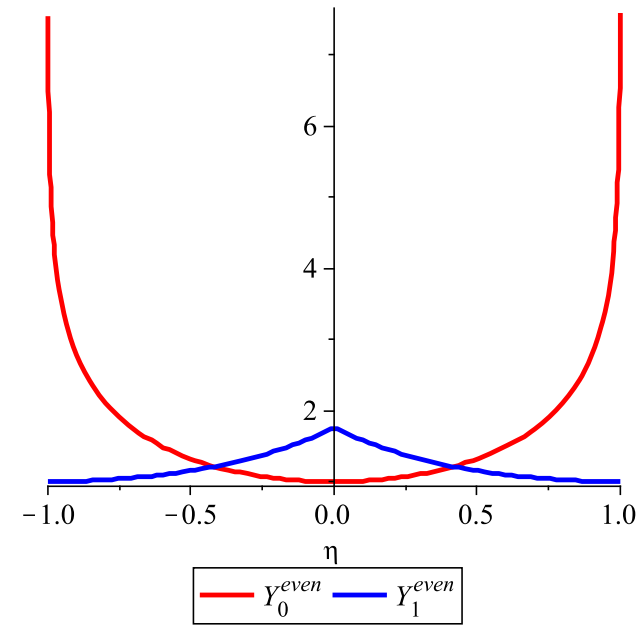

(b) Linearly independent

Figure 2.3: The two angular confluent Heun functions showing the contrasting linear dependence explicitly. In the first case $\left(\lambda=2.20527, \mu_{00}(\lambda)=0.8117302\right)$ the correct $\mu_{00}(\lambda)$ results in linear dependence, whereas the second case $(\lambda=$ $2.20527, \mu=2.20527$ ) an incorrect $\mu$ results in linear independence.

(The separation parameter in $[30,31]$ is -1 times our separation parameter.)

The Wronskian result suggests that if the confluent Heun functions were expressed in terms of elementary functions an analytic expression for $\mu_{m n}(\lambda)$ might be obtainable. That is, if we can determine $F(\mu, \lambda)$, then the zeros of this function will give $\mu_{m n}(\lambda)$. Eqn. 2.40 is not of the hypergeometric form, however, attempts were made to reduce it to a simpler form, whose solution would be in terms of elementary transcendental functions. However, it was only possible to derive the Wronskian in terms of the confluent Heun functions.

We may exhibit the contrasting linear dependence of the two angular confluent Heun solutions by plotting them for a given $\lambda$ with the correct $\mu_{m n}(\lambda)$, and in the other case an incorrect $\mu$. This is shown in Fig. 2.3. The two angular solutions are linearly dependent when $\mu=\mu_{00}(\lambda)$, but are linearly independent when $\mu \neq \mu_{00}(\lambda)$. The contrasting linear dependence of the angular solutions determining whether or not the correct $\mu_{m n}(\lambda)$ separation parameter is obtained is the principle behind the Wronskian. 


\subsubsection{The radial Wronskian}

In section 2.4.1 we derived the $\mu_{m n}(\lambda)$ and $\lambda$ relation through an angular Wronskian. In order to derive the total energy of $\mathrm{H}_{2}^{+}$the relation between $R(\lambda)$ and $\lambda$ is required. This section shall investigate the possible extension of the Wronskian method to the radial case to deduce the $R(\lambda)$ relation. The Wronskian method applied in the radial case is mathematically represented in the form of the following expression

$$
W_{R}(R, \lambda, \xi)=0 \Leftrightarrow R=R(\lambda),
$$

where the $\mu$ dependence has been removed by solving an angular Wronskian for $\mu_{m n}(\lambda)$. The radial equation, as derived previously, is given by

$$
\frac{d}{d \xi}\left\{\left(\xi^{2}-1\right) \frac{d X}{d \xi}\right\}+\left(-\lambda \xi^{2}+2 R \xi-\frac{m^{2}}{\xi^{2}-1}+\mu\right) X=0 .
$$

Eqn. 2.49 is a radial equation due to the fact that the variable $\xi$ is of infinite extent. Following the Wronskian method in the angular case, two solutions to the radial equation which are linearly dependent when $R=R(\lambda)$ are sought.

The radial equation has two regular singularities at the points $\xi=-1$ and $\xi=1$, and an irregular singularity at $\infty$. However, the point $\xi=-1$ is not physical and corresponds to a negative distance. Nonetheless, there are two solutions to the radial equation (for $m=0$ ), one non-singular at the regular singular point $\xi=1$, and the other non-singular at the regular singular point $\xi=-1$, given by

$$
\begin{gathered}
X_{1}(\xi)=e^{-\sqrt{\lambda}(\xi-1)} \operatorname{HeunC}\left(4 \sqrt{\lambda}, 0,0,-4 R, 2 R-\lambda+\mu, \frac{-\xi+1}{2}\right), \\
X_{-1}(\xi)=e^{\sqrt{\lambda}(\xi+1)} \operatorname{HeunC}\left(4 \sqrt{\lambda}, 0,0,4 R,-2 R-\lambda+\mu, \frac{\xi+1}{2}\right) .
\end{gathered}
$$

$X_{-1}$ is non-singular at $\xi=-1$, but it is singular at $\xi=1$ for arbitrary eigenvalue parameters. In order to extend this solution into the physical range of $\xi$ analytic continuation must be performed. However, this leads to a solution which is now complex and has both real and imaginary components. The two radial solutions are shown in Fig. 2.4, in one case with the correct triple $\left(R(\lambda), \mu_{00}(\lambda), \lambda\right)$, and in the other case an incorrect triple $(R, \mu, \lambda)$. 


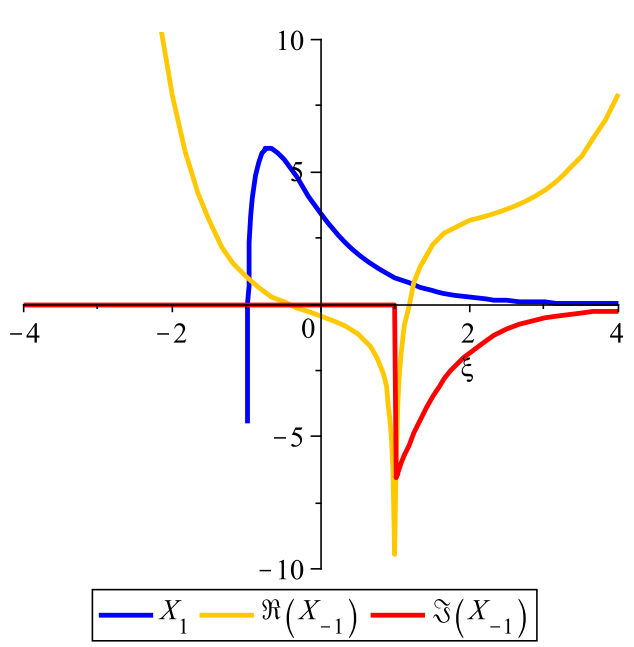

(a) Linearly independent

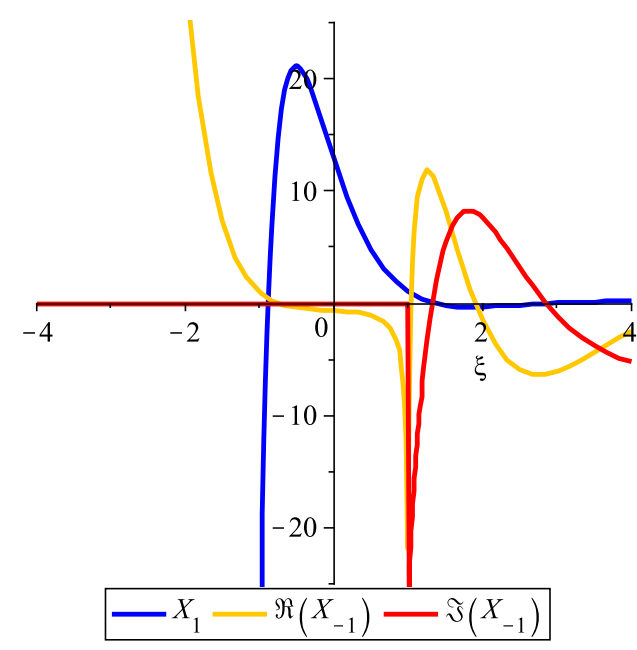

(b) Linearly independent

Figure 2.4: The two radial solutions showing the contrasting linear dependence explicitly. In the first case $(R=2, \mu=0.8117302, \lambda=2.20527)$ the correct $\left(R(\lambda), \mu_{00}(\lambda), \lambda\right)$ results in linear independence. In the second case $(R=5, \mu=$ $0.8117302, \lambda=2.20527)$ an incorrect $(R, \mu, \lambda)$ also results in linear independence. The linear independence does not permit the Wronskian method to be applied to these two solutions.

The two radial solutions are linearly independent and so their Wronskian may or may not be zero when $R=R(\lambda)$. The Wronskian equal to zero gives a necessary condition for linear dependence, thus, it is possible that even though they are linearly independent their Wronskian is equal to zero. In this case, however, the Wronskian is non-zero when $R=R(\lambda)$ and so the method which was applicable in the angular case cannot be applied here to these two solutions.

The Wronskian of two solutions to the radial equation may be derived using Abel's identity, as was the case for the angular equation. By Abel's identity, the Wronskian in the radial case is of the form

$$
W_{R}(R, \lambda, \xi)=\frac{F(R, \lambda)}{\xi^{2}-1} .
$$

There is no $\mu_{m n}(\lambda)$ dependency in this expression. Since $\mu_{m n}(\lambda)$ is a function of $\lambda$ it may be approximated through a series representation, or obtained exactly through the zeros of an angular Wronskian. 
It is interesting that Eqn. 2.52 has the same form as Eqn. 2.47 in the angular case. Although the form of the Wronskian is known in the radial case, we do not have the required two linearly dependent solutions needed to obtain the Wronskian function $F(R, \lambda)$. We have one exact solution of the radial equation, $X_{1}$, which is finite in the range of $\xi$. Therefore, an area of future research would be to investigate the possibility of a second solution to the radial equation, which is linearly dependent with $X_{1}$ when $R=R(\lambda)$. The solution to the radial equation, about the irregular singular point $\xi=\infty$, would be linearly dependent with $X_{1}$, when $R=R(\lambda)$, but no closed form expression for this solution has been obtained.

\subsection{The radial equation}

Section 2.4.1 derived a method which gives the exact $\mu_{m n}(\lambda)$ and $\lambda$ relation. The exact $\mu_{m n}(\lambda)$ is given by the zeros of the Wronskian of two linearly dependent angular solutions. However, it was not possible to extend this method to the radial case. Although two solutions to the radial equation were found, they are not linearly dependent when $R=R(\lambda)$. In this section the method of solving the radial equation, as derived by Jaffé [6], shall be presented, along with a discussion of the method of other researchers. This method accurately determines the ground state energy of $\mathrm{H}_{2}^{+}$and is in agreement with experimental results.

The radial equation for $\mathrm{H}_{2}^{+}$is given in Eqn. 2.49. Following Jaffé, we make the following substitution in the radial equation

$$
X(\xi)=\left(\xi^{2}-1\right)^{m / 2} f(\xi)
$$

The substitution in Eqn. 2.53 gives the following differential equation for $f(\xi)$

$$
\left(\xi^{2}-1\right) f^{\prime \prime}+2 \xi(m+1) f^{\prime}+\left(2 R \xi-\lambda \xi^{2}+m^{2}+m+\mu\right) f=0 .
$$

The prime denotes differentiation with respect to $\xi$. 
Now let $f(\xi)$ have the following form

$$
f(\xi)=e^{-\sqrt{\lambda} \xi}(\xi+1)^{R / \sqrt{\lambda}-1-m} w(u), \quad u=\frac{\xi-1}{\xi+1} .
$$

Performing the substitution of Eqn. 2.55 leads to a differential equation for $w(u)$, namely

$$
\begin{array}{r}
u(1-u)^{2} w^{\prime \prime}+\left[-(m+2 \sigma-1) u^{2}+2(\sigma-2 p-1) u+m+1\right] w^{\prime} \\
+[\sigma(\sigma+m) u+\sigma(1+2 p)+m(m+1+\sigma)+\mu-\lambda] w=0,
\end{array}
$$

where the following simplifying substitutions have been made

$$
\begin{aligned}
\sigma & =R / \sqrt{\lambda}-1-m, \\
p & =\sqrt{\lambda} .
\end{aligned}
$$

The point $u=0$ corresponds to $\xi=1$ in the original radial equation. Therefore, the differential equation for $w(u)$ can be solved by means of a power series about the origin:

$$
w(u)=\sum_{n=0}^{\infty} c_{n} u^{n} .
$$

As is the case with most series solutions of confluent Heun equations, the coefficients $c_{n}$ satisfy a three term recurrence relation:

$$
\begin{array}{r}
(n+1)(n+m+1) c_{n+1}+(n-1-\sigma)(n-1-\sigma-m) c_{n-1} \\
-\left[2 n^{2}+(4 p-2 \sigma) n-\mu+\lambda-2 p \sigma-(m+1)(m+\sigma)\right] c_{n}=0 .
\end{array}
$$

Eqn. 2.59 is a second-order difference equation of the Poincaré-Perron type [34]. To investigate the convergence of the series solution proposed in Eqn. 2.58 , we study the difference equation in the case of large $n$.

For large $n$, we assume the ratio of consecutive coefficients has the following form

$$
\frac{c_{n+1}}{c_{n}}=1+\frac{a}{\sqrt{n}}+O\left(\frac{1}{n}\right) .
$$

Inserting Eqn. 2.60 into Eqn. 2.59 we find

$$
a^{2}=4 p .
$$


It follows that

$$
\frac{c_{n+1}}{c_{n}}=1 \pm \sqrt{\frac{4 p}{n}}+O\left(\frac{1}{n}\right) .
$$

Provided we take the solution of Eqn. 2.59 whose coefficients behave asymptotically as $\frac{c_{n+1}}{c_{n}}=1-\sqrt{\frac{4 p}{n}}$, then the series solution in Eqn. 2.58 is convergent for $0 \leq u \leq 1$.

The recurrence relation of Eqn. 2.59 can be expressed as

$$
c_{n+1}+u_{n} c_{n}-v_{n-1} c_{n-1}=0, \quad n=0,1,2, \ldots,
$$

where $c_{-1}=0$ and $u_{n}, v_{n-1}$ are

$$
\begin{aligned}
u_{n} & =-\frac{2 n^{2}+(4 p-2 \sigma) n-\mu+\lambda-2 p \sigma-(m+1)(m+\sigma)}{(n+1)(n+m+1)}, \\
v_{n-1} & =-\frac{(n-1-\sigma)(n-1-\sigma-m)}{(n+1)(n+m+1)} .
\end{aligned}
$$

For the series solution in Eqn. 2.58 to be convergent, we require that the coefficients $c_{n}$ tend to zero as $n$ tends to $\infty$. This gives the desired transcendental expression, relating $R(\lambda), \mu(\lambda)$, and $\lambda$. Therefore by setting $c_{N}=0$ in Eqn. 2.63, as $N \rightarrow \infty$, and incorporating the series representation for $\mu_{00}(\lambda)$, we shall obtain a relation which determines $R(\lambda)$, and hence the total energy of $\mathrm{H}_{2}^{+}$. Setting $c_{N}=0$ allows the transcendental relationship between $R(\lambda), \mu(\lambda)$, and $\lambda$ to be expressed as an infinite continued fraction. The infinite continued fraction to be solved to give the $R(\lambda)$ relation is given by

$$
-u_{0}=\frac{v_{0}}{u_{1}+} \frac{v_{1}}{u_{2}+} \frac{v_{2}}{u_{3}+}+\ldots
$$

This is a transcendental relation for the parameters $R(\lambda), \mu(\lambda)$, and $\lambda$. The relation in Eqn. 2.66, along with the $\mu_{00}(\lambda)$ series, determines $R(\lambda)$ and thus the total energy of the hydrogen molecule ion.

The method of solution of Jaffé is interesting and deserves further comment. Jaffé follows a similar procedure to that outlined by Wilson. However, he performs a Jaffé transformation of the independent variable first $(u=(\xi-1) /(\xi+1))$, and then performs the series expansion of the solution about the point $\xi=1$. The asymptotic solution of the recurrence relation for the coefficients in the series shows that the series is convergent, and that the infinite continued fraction is convergent. 
The method of Jaffé is more general than just the $\mathrm{H}_{2}^{+}$problem. It gives a method of solving the central two point connection problem in mathematics. That is, given the asymptotic nature of the solution at $\infty$, and the series solution about the origin, what are the eigenvalue parameters in order that these two solutions match up? [34], [35].

The solution proposed by Hylleraas [7] is of a different type to that just mentioned. Hylleraas gave a solution to the radial equation as an expansion of associated Laguerre polynomials multiplied by an exponential function. This led to a three term recurrence relation for the series coefficients. The three term recurrence relation gives an infinite continued fraction, which is convergent, and allows the ground state solution to be obtained.

Our treatment in this section follows closely that of Baber and Hassé [36], who have rigourously shown that by writing the solution to Eqn. 2.59 as an infinite continued fraction leads to the required transcendental relation. Baber and Hassé discuss the question of convergent and divergent radial solutions in more detail and, in contrast to Jaffé's statement, show that Hylleraas's radial series solution is convergent.

The first paper to treat the $\mathrm{H}_{2}^{+}$problem using wave mechanics was that of Burrau [3]. Burrau's method of solving the angular and radial equation is equivalent. In the angular case Burrau turns the second-order equation into a non-linear first-order Riccati differential equation. By power series expansion of the solution at $\eta=0$, and the solution at $\eta=1$, Burrau finds that the two solutions coincide when $\mu=\mu(\lambda)$. The method of solution of the radial equation is to also convert the second-order differential equation into a non-linear first-order Riccati differential equation. Burrau then performs an asymptotic expansion about the irregular singular point, and finds that this solution matches the series solution about the point $\xi=1$, when $R=R(\lambda)$. The solution of Burrau has, however, been criticized by Wilson [4] as an asymptotic solution.

The introduction of Flammer's book [31] gives references to further research on the $\mathrm{H}_{2}^{+}$problem which has been performed following the pioneering work of Burrau, Hylleraas, and Wilson and Jaffé. 


\subsection{The ground state of the hydrogen molecule ion}

Section 2.5 derived a transcendental relation which relates $R(\lambda), \mu_{00}(\lambda)$, and $\lambda$. Using this relation, along with the $\mu_{00}(\lambda)$ series, allows $R(\lambda)$ and hence the electron energy of $\mathrm{H}_{2}^{+}$to be determined. In this section we shall present the ground state equilibrium parameters of $\mathrm{H}_{2}^{+}$, as derived by Jaffé, and compare with the results of Hylleraas. (The results obtained by Jaffé and Hylleraas for the ground state energy use the series solution for $\mu_{00}(\lambda)$. Thus all calculations in this section use the $\mu_{00}(\lambda)$ series value, which agrees with the Wronskian result.)

By solving Eqn. 2.66 for $R(\lambda)$ the total energy of $\mathrm{H}_{2}^{+}$may be deduced using Eqn. 2.13. Table 2.1 gives the total energy of $\mathrm{H}_{2}^{+}$in [au], for various values of the internuclear separation $R(\lambda)$, as computed by both Jaffé and Hylleraas.

\begin{tabular}{|r|c|c|}
\hline \multicolumn{3}{|c|}{$\mathrm{H}_{2}^{+}$total energy } \\
\hline$R$ & Jaffé & Hylleraas \\
\hline 0.5 & 0.2650 & 0.2651 \\
1 & -0.45175 & -0.4523 \\
2 & -0.60264 & -0.602635 \\
3 & -0.57722 & -0.57755 \\
\hline
\end{tabular}

Table 2.1: The total energy (electron plus internuclear) of $\mathrm{H}_{2}^{+}$, for fixed nuclei, as computed by Jaffé and Hylleraas.

Table 2.1 shows excellent agreement between the methods of Jaffé and Hylleraas. Fig. 2.5 shows a plot of the total energy as a function of the internuclear separation $R(\lambda)$. The total energy shown in Fig. 2.5 has a minimum at a nuclear separation of $R_{e}=2 a_{0}$, where $a_{0}$ is the Bohr radius, i.e. $R_{e}=1.06 \AA$, which corresponds to the stable equilibrium configuration. For distances significantly larger than this the energy is that of a hydrogen atom and a free proton. At a distance smaller than the equilibrium separation length there is a larger repulsion of the two protons, and as a result does not correspond to a stable molecular state. 


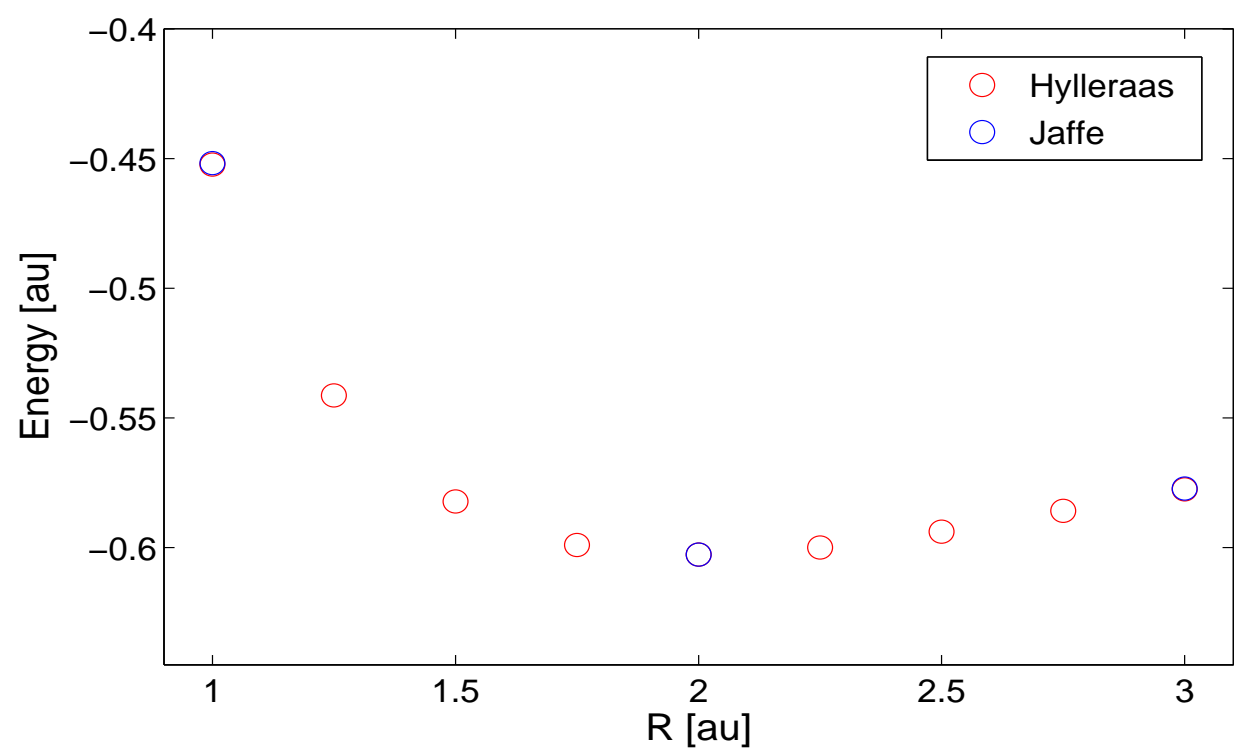

Figure 2.5: The total energy (electron plus internuclear) of $\mathrm{H}_{2}^{+}$, for fixed nuclei, as a function of the internuclear separation.

The total energy of $\mathrm{H}_{2}^{+}$may be computed for various nuclear separations, using either the method of Jaffé or Hylleraas. However, an analytic expression for the total energy is at the moment unavailable. Therefore, we must represent the total energy, as a function of the nuclear separation, by a suitable approximation. Using this energy, as in the Born-Oppenheimer approximation, the nuclear wave equation may be solved with the total energy term playing the role of the potential. This is now reduced to a familiar two-body problem, and consequently two one-body problems. One describes the centre of mass moving as a free particle, with the mass of the combined protons, and the other describes the two protons moving effectively as a particle, with the reduced mass, in the field of the total energy. The motion of the reduced mass, as opposed to the translational motion of the centre of mass, is of most interest.

The asymmetrical nature of the total energy is evident on the scale of Fig. 2.5. As discussed previously, a suitable approximation is needed to represent the total energy, which would subsequently allow the nuclear wave equation to be solved. Such a potential is the Morse potential. 
By matching a Morse curve to the total energy, Hylleraas has obtained an expression for the energy levels of the hydrogen molecule ion. The theory of the Morse potential may be found in Chapter 10 of [8], which we shall omit for brevity.

The energy levels of the hydrogen molecule ion (electron and internuclear energy plus nuclear vibrational energy), for the Morse fit to the total energy of Hylleraas, is:

$$
E_{n}^{M}=-0.602635+0.0103\left(n+\frac{1}{2}\right)-0.000255\left(n+\frac{1}{2}\right)^{2}, n=0,1,2, \ldots
$$

The energy levels derived by Hylleraas are in agreement with those obtained experimentally by extrapolation of the observed vibrational levels for excited states of the hydrogen molecule. The coefficients in Eqn. 2.67 are found by Birge [37], and Richardson [38], to be 0.0104 and -0.00028 , and 0.0105 and -0.000275 respectively.

The ground state equilibrium parameters of $\mathrm{H}_{2}^{+}$obtained by Hylleraas are given in Table 2.2 .

\begin{tabular}{|r|c|}
\hline \multicolumn{2}{|c|}{$\mathrm{H}_{2}^{+}$ground state parameters } \\
\hline$R_{e}$ & $1.06 \AA$ \\
$E_{\text {vib }}$ & $0.138404 \mathrm{eV}$ \\
$E_{\text {min }}$ & $-16.398542 \mathrm{eV}$ \\
$D_{0}$ & $2.654438 \mathrm{eV}$ \\
\hline
\end{tabular}

Table 2.2: The equilibrium parameters in the ground state of $\mathrm{H}_{2}^{+}$. These are, respectively, the equilibrium nuclear separation, the ground state vibrational energy, the minimum total energy, and the dissociation energy of the molecule.

The ground state energy of the hydrogen molecule ion is $E_{g}=-16.2601 \mathrm{eV}$ $\approx-0.59755 \mathrm{au}$. The dissociation energy in the lowest vibrational state, into a free proton and a hydrogen atom, is $D_{0}=2.6544 \mathrm{eV} \approx 0.09755 \mathrm{au}$, which indicates the relative strength of the bond of the molecule. In conclusion, the methods of Jaffé and Hylleraas give an accurate description of the ground state of $\mathrm{H}_{2}^{+}$, which is in agreement with experiment. 
The solution to the angular and radial equations now permit the electronic wave function for the ground state of $\mathrm{H}_{2}^{+}$to be constructed. From the expressions for $X(\xi)$ and $Y(\eta)$ in Eqn. 2.50 and Eqn. 2.44, we obtain the ground state wave function of the electron as

$$
\begin{aligned}
\psi(\xi, \eta)= & e^{-\sqrt{\lambda}(\xi-1)} \operatorname{HeunC}\left(4 \sqrt{\lambda}, 0,0,-4 R, 2 R-\lambda+\mu, \frac{-\xi+1}{2}\right) \\
& \times \operatorname{HeunC}\left(0,0,-\frac{1}{2}, \frac{\lambda}{4}, \frac{\mu-\lambda+1}{4}, 1-\eta^{2}\right) .
\end{aligned}
$$

The ground state probability density of the electron, along the line joining the nuclei, is shown in Fig. 2.6. Fig. 2.7 shows a contour plot of the probability density in two dimensions. Fig. 2.6 shows that the probability density is large in the region between the nuclei and sufficiently small far away from them. This expresses the fact that the electron is in essence shared between the two protons and it is in this stable configuration for which the proton repulsion is balanced by the electron-proton attraction. This quantum mechanical argument demonstrates the existence of the oneelectron covalent bond in $\mathrm{H}_{2}^{+}$, and shows that the ground state corresponds to an actual bound state. In the next sections we shall see that only the ground state of $\mathrm{H}_{2}^{+}$exhibits binding. 


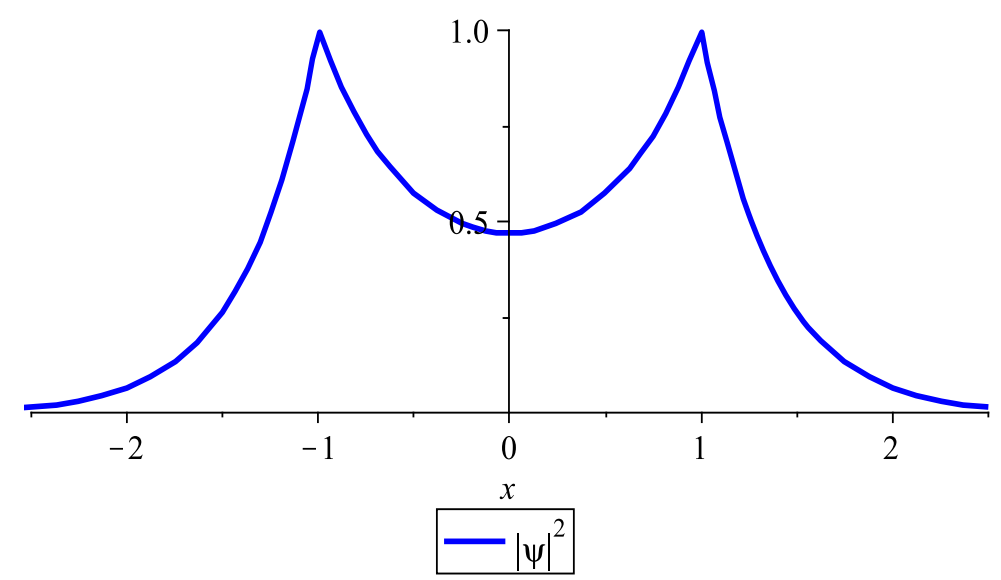

Figure 2.6: $|\psi|^{2}$ along the line joining the nuclei. The variable $x$ is the distance (along the line of the nuclei) from the midpoint of the two nuclei to the electron. The two nuclei are located at $x=-1$ and $x=1$, where the units of $x$ are the Bohr radius $a_{0}$. The electron is largely confined in a region between the two protons, resulting in a stable bond.

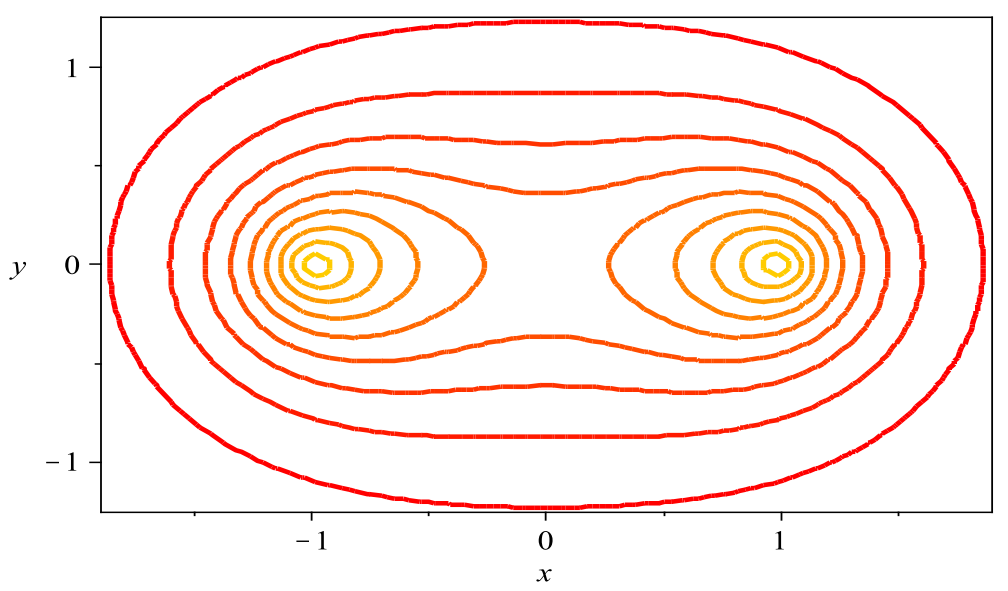

Figure 2.7: Contour plot of $|\psi|^{2}$. The variable $y$ is the distance (perpendicular to the line of the nuclei) from the line of the nuclei to the electron. The two nuclei are located at $x=-1$ and $x=1$, where the units of $x$ and $y$ are the Bohr radius $a_{0}$. The contour levels are $0.1,0.2, \ldots, 0.9$. Fig. 2.6 is along the line $y=0$. 


\subsection{Anti-bonding excited state}

The ground state of $\mathrm{H}_{2}^{+}$is an even (gerade) state denoted by ${ }^{2} \Sigma_{g}^{+}$. In this section an excited state of $\mathrm{H}_{2}^{+}$shall be investigated. The excited state has zero angular momentum along the nuclear axis $(m=0)$ and an odd (ungerade) electron wave function. In the terminology introduced in section 2.2 this state is denoted by ${ }^{2} \Sigma_{u}^{+}$. By solving the angular equation for an odd angular confluent Heun function, and using the radial solution $X_{1}$, a wave function odd in $\eta$ will be obtained. Two odd solutions to the angular equation $(m=0)$ are given by the following expressions

$$
\begin{aligned}
& Y_{0}^{\text {odd }}(\eta)=\eta \operatorname{HeunC}\left(0, \frac{1}{2}, 0,-\frac{\lambda}{4}, \frac{\mu+1}{4}, \eta^{2}\right) \\
& Y_{1}^{\text {odd }}(\eta)=\eta \operatorname{HeunC}\left(0,0, \frac{1}{2}, \frac{\lambda}{4}, \frac{\mu-\lambda+1}{4}, 1-\eta^{2}\right) .
\end{aligned}
$$

As in section $2.4, \mu_{o}(\lambda)$ shall denote $\mu_{0 n}(\lambda)(n=1,3,5, \ldots)$, i.e. the odd physical separation parameters. These two solutions are linearly dependent solutions when $\mu=\mu_{o}(\lambda)$, but are linearly independent for an arbitrary $\mu$. Therefore, the Wronskian of these two solutions can be used to determine the separation parameter $\mu_{o}(\lambda)$ as a function of $\lambda$. The odd angular Wronskian in this case is given by

$$
\begin{array}{r}
W_{A}^{\text {odd }}(\mu, \lambda, \eta)=-2 \eta^{3}\left\{\left[\operatorname{HeunC}\left(0,0, \frac{1}{2}, \frac{\lambda}{4}, \frac{\mu-\lambda+1}{4}, 1-\eta^{2}\right)\right.\right. \\
\left.\times \text { HeunCPrime }\left(0, \frac{1}{2}, 0,-\frac{\lambda}{4}, \frac{\mu+1}{4}, \eta^{2}\right)\right]+\left[\operatorname{HeunC}\left(0, \frac{1}{2}, 0,-\frac{\lambda}{4}, \frac{\mu+1}{4}, \eta^{2}\right)\right. \\
\left.\left.\times \operatorname{HeunCPrime}\left(0,0, \frac{1}{2}, \frac{\lambda}{4}, \frac{\mu-\lambda+1}{4}, 1-\eta^{2}\right)\right]\right\} .
\end{array}
$$

Solving Eqn. 2.71 equal to zero, for example with $\eta=1 / \sqrt{2}$, will determine the exact relation between $\mu_{o}(\lambda)$ and a given $\lambda$, as was the case with the even Wronskian. The $\mu_{o}(\lambda)$ separation parameter will be different from the $\mu_{e}(\lambda)$ separation parameter, but a series representation may also be found by following the procedure given in section 2.3 , only with $l$ odd starting from $l=1$ now. The series representation in this case, for the lowest $\mu_{o}(\lambda)$, is given by

$$
\mu_{01}(\lambda)=-2+\frac{3}{5} \lambda+\frac{6}{875} \lambda^{2}-\frac{4}{65625} \lambda^{3}+O\left(\lambda^{4}\right)
$$




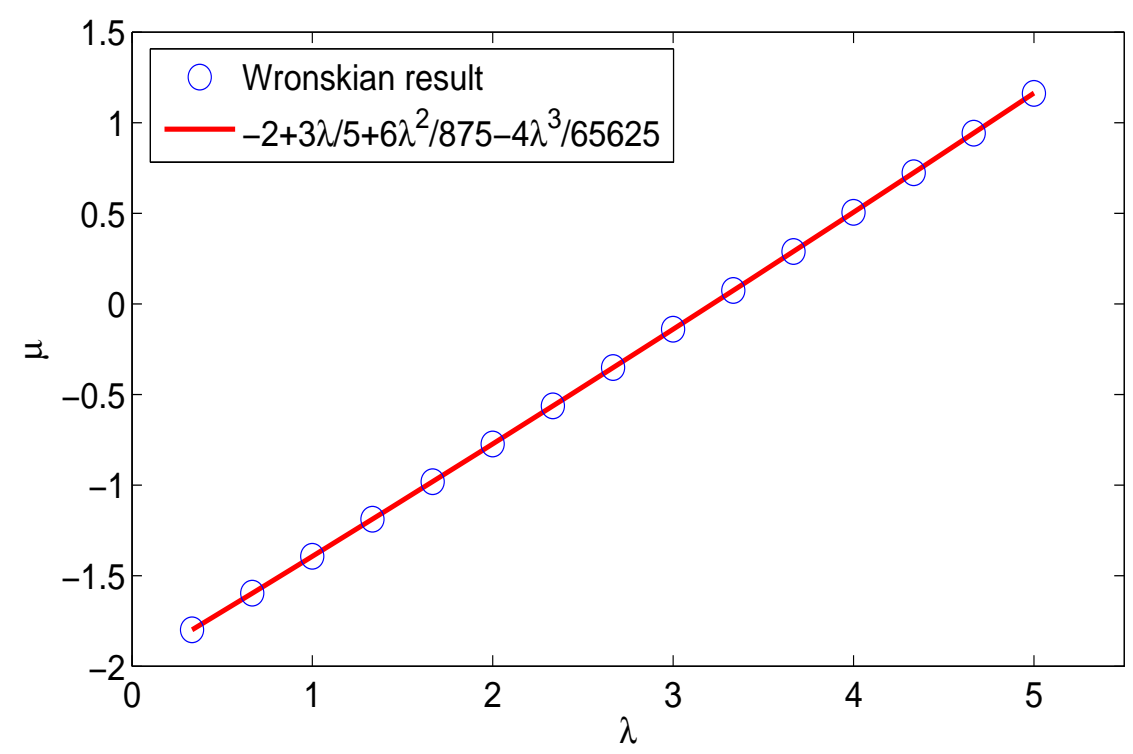

Figure 2.8: Comparison between the $\mu_{01}(\lambda)$ value computed using the Wronskian method and with the series method for a range of $\lambda$ values.

Comparison between the series solution and the results found using the Wronskian are shown in Fig. 2.8. Again the Wronskian method deduces the correct $\mu_{o}(\lambda)$ which is in agreement with the series solution obtained in Eqn. 2.72. Thus, in both the even and odd cases, we have derived an exact, transcendental equation that gives the separation parameter. In both the even and odd cases the roots of a single equation give the values of all the physically allowed separation parameters. As in Eqn. 2.47 we may derive the Wronskian analytically using Abel's identity:

$$
W_{A}^{\text {odd }}(\mu, \lambda, \eta)=\frac{F^{o d d}(\mu, \lambda)}{1-\eta^{2}} .
$$

The Wronskian function, $F^{o d d}(\mu, \lambda)$, is zero at one of the physical values $\mu_{0 n}(\lambda), n=1,3,5, \ldots$ For example, for $\lambda=1$, the odd $\mu_{0 n}(\lambda)$ values are approximately $-1.393206,-11.492120, \ldots$ These values may be compared with those in $[30,31]$ which are equivalent (the separation parameter in $[30,31]$ is -1 times our separation parameter.) 


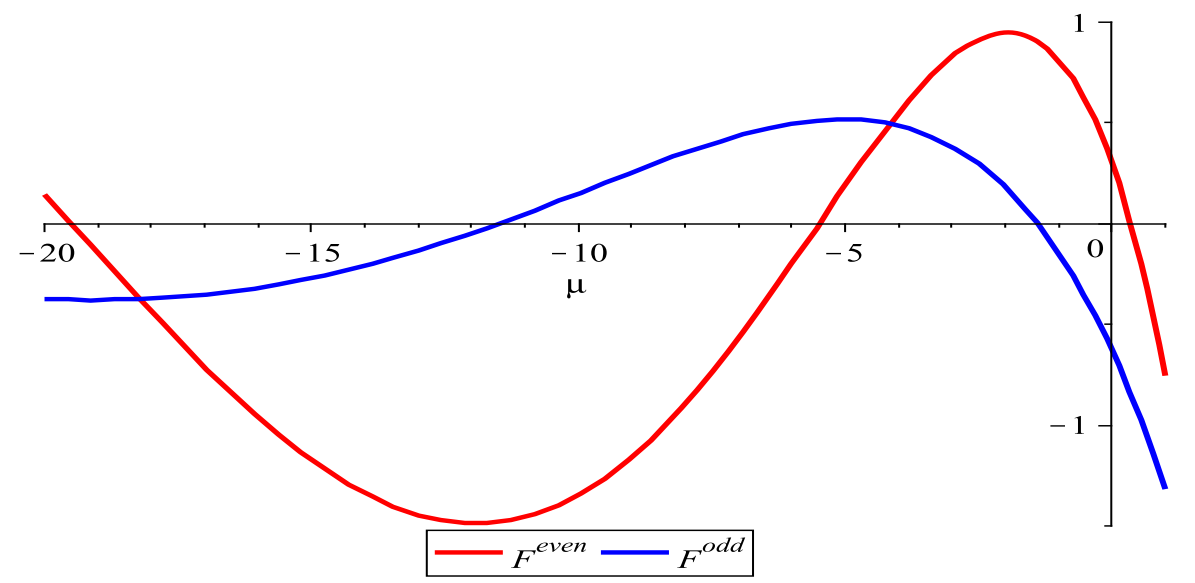

Figure 2.9: The Wronskian functions, $F^{\text {even }}(\mu, \lambda)$ and $F^{o d d}(\mu, \lambda)$, plotted for $\lambda=1$. The Wronskian functions are dependent on $\mu$, and each zero corresponds to a different physically allowed $\mu_{0 n}(\lambda)$. In the even case the first few zeros correspond to $\mu_{00}, \mu_{02}, \mu_{04}$, while in the odd case the zeros correspond to $\mu_{01}, \mu_{03}$.

The two Wronskian functions, even and odd cases, are plotted as functions of $\mu$ for a fixed $\lambda$ in Fig. 2.9. Fig. 2.9 shows that the zeros of the Wronskian functions, in both the even and odd cases, correspond to the physical separation parameters.

Obtaining $\mu_{01}(\lambda)$ allows $R(\lambda)$ to be determined by solving the radial equation, as was the case in the ground state. This then allows the total energy to be obtained as a function of the internuclear separation. The total energy of $\mathrm{H}_{2}^{+}$in the ${ }^{2} \Sigma_{u}^{+}$has been obtained by Bates et al. [39], who use Jaffé's method of solution for the radial equation, and the series value for $\mu_{01}(\lambda)$. Fig. 2.10 shows the total energy for this excited state of $\mathrm{H}_{2}^{+}$. In this state the total energy is repulsive for all internuclear separations, consequently it does not correspond to a bound state.

We can physically understand why the ${ }^{2} \Sigma_{u}^{+}$state corresponds to an anti-bonding state by determining the probability density. The probability density for the anti-bonding state is shown in Fig. 2.11 and shows completely contrasting behaviour when compared with the bonding ground state. In the ground state the probability density is large in the region between the two nuclei. In the excited state ${ }^{2} \Sigma_{u}^{+}$, however, the probability density is confined in the region around the two nuclei. 


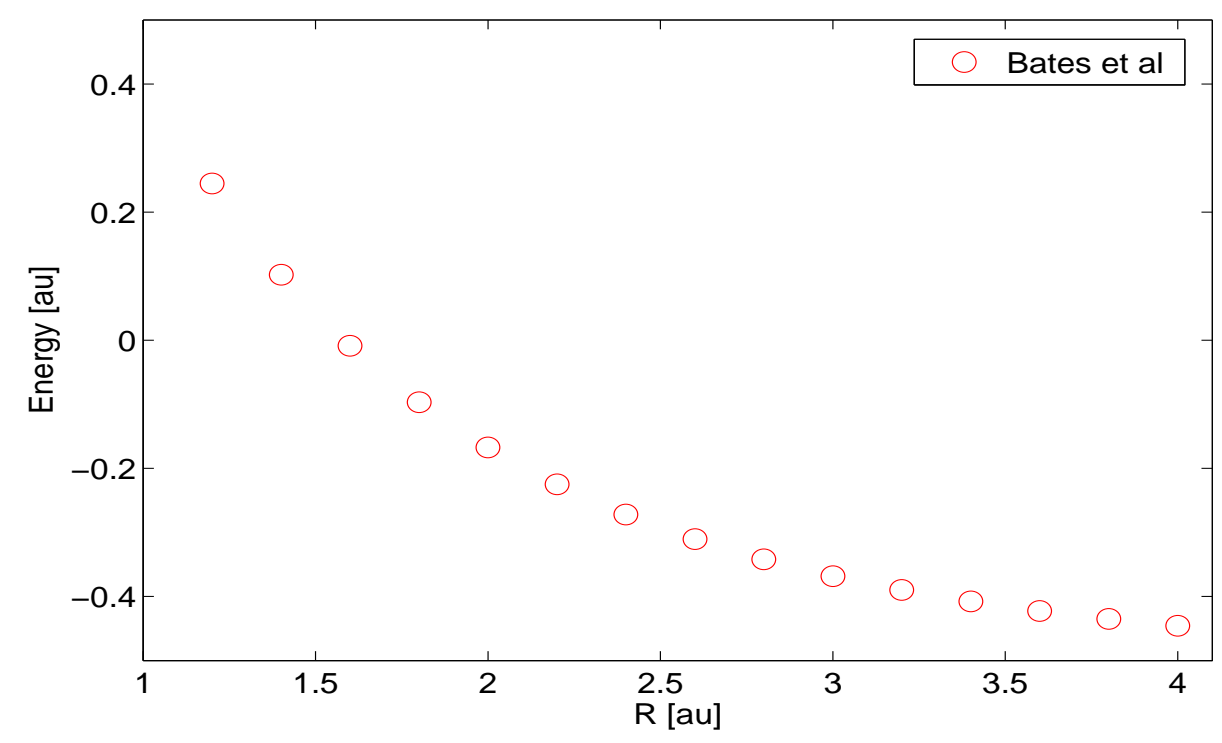

Figure 2.10: The total energy of $\mathrm{H}_{2}^{+}$in the ${ }^{2} \Sigma_{u}^{+}$state, as a function of the nuclear separation. The energy is monotonically decreasing in this range and always lies above $-0.5 \mathrm{au}$, resulting in an unbound system. There is a small minimum when $R=12.6 a_{0}$ due to Van der Waal forces, but the energy is $-5.8 \times 10^{-5} \mathrm{au}$, which on the scale of the ground state energy is negligible [29]. (This state is labelled as $2 p \sigma_{u}$ in the notation of [39].)

The presence of the extra node in the probability density also results in an increase in the total energy, as confirmed in Fig. 2.10. These two facts show that the electron in this excited state is not shared between the two nuclei and is more likely to be bound to one of the protons. There is no evidence of a sharing of the electron in this instance, and consequently a free proton and a hydrogen atom are more stable than this configuration. This indicates that, on excitation from the ground state to this (virtual) excited state, $\mathrm{H}_{2}^{+}$will readily dissociate into a free proton and a hydrogen atom.

In conclusion, this section has demonstrated the applicability of the Wronskian method to determine $\mu_{0 n}(\lambda)$ exactly, where $n$ is odd. The next section shall generalize the Wronskian method to the case of arbitrary $m$. 


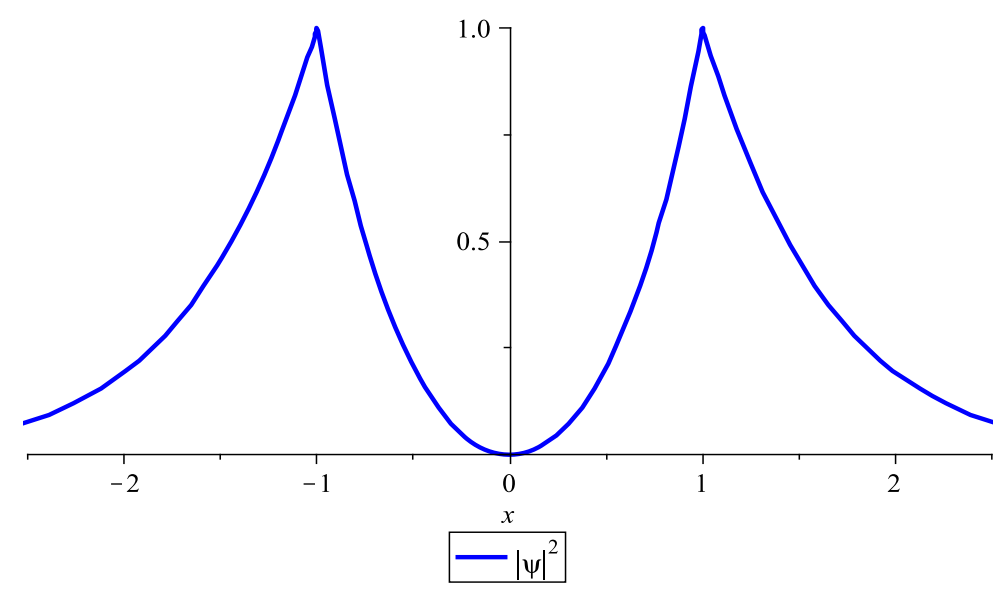

Figure 2.11: $|\psi|^{2}$ along the line joining the nuclei. The variable $x$ is the distance (along the line of the nuclei) from the midpoint of the two nuclei to the electron. The two nuclei are located at $x=-1$ and $x=1$, where the units of $x$ are the Bohr radius $a_{0}$. The node in the center of the nuclear axis results in an energy larger than the ground state energy leading to instability.

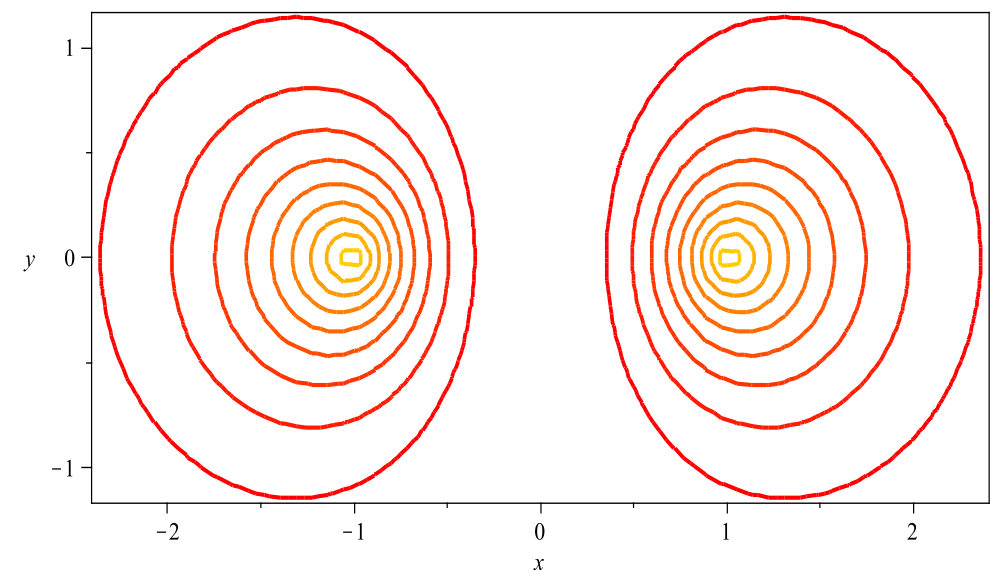

Figure 2.12: Contour plot of $|\psi|^{2}$. The variable $y$ is the distance (perpendicular to the line of the nuclei) from the line of the nuclei to the electron. The two nuclei are located at $x=-1$ and $x=1$, where the units of $x$ and $y$ are the Bohr radius $a_{0}$. The contour levels are $0.1,0.2, \ldots, 0.9$. Fig. 2.11 is along the line $y=0$. 


\subsection{Non-zero orbital angular momentum}

We consider here the general solutions to Eqn. 2.39, and extend the Wronskian method to states with non-zero $m$ (angular momentum along the nuclear axis). In the angular case, the Wronskian gives an exact transcendental relation between the separation parameter $\mu_{m n}(\lambda)$ and $\lambda$.

As in section 2.4.1, we seek to determine the $\mu_{m n}(\lambda)$ dependency by solving the angular equation. This may be done using even or odd linearly dependent solutions via the zeros of the Wronskian. For the even case, the angular equation has the following solutions

$$
\begin{aligned}
& Y_{1}^{\text {even }}(\eta)=\left(1-\eta^{2}\right)^{m / 2} \operatorname{HeunC}\left(0, m,-\frac{1}{2}, \frac{\lambda}{4}, \frac{\mu-\lambda+1+m^{2}}{4}, 1-\eta^{2}\right), \\
& Y_{0}^{\text {even }}(\eta)=\left(1-\eta^{2}\right)^{m / 2} \operatorname{HeunC}\left(0,-\frac{1}{2}, m, \frac{-\lambda}{4}, \frac{\mu+1+m^{2}}{4}, \eta^{2}\right) .
\end{aligned}
$$

In the odd case two solutions to the angular equation are given by

$$
\begin{aligned}
& Y_{1}^{\text {odd }}(\eta)=\left(1-\eta^{2}\right)^{m / 2} \eta \operatorname{HeunC}\left(0, m, \frac{1}{2}, \frac{\lambda}{4}, \frac{\mu-\lambda+1+m^{2}}{4}, 1-\eta^{2}\right), \\
& Y_{0}^{\text {odd }}(\eta)=\left(1-\eta^{2}\right)^{m / 2} \eta \operatorname{HeunC}\left(0, \frac{1}{2}, m,-\frac{\lambda}{4}, \frac{\mu+1+m^{2}}{4}, \eta^{2}\right) .
\end{aligned}
$$

These solutions enable the Wronskian method to be applied for the case of arbitrary $m$ to deduce the $\mu_{m n}(\lambda)$ dependency. We shall illustrate this only in the $m=1$ case. Solving the Wronskian of the two odd solutions in Eqns. 2.75 equal to zero (for a given $\lambda$ ) will give the set $\mu_{1 n}(\lambda),(n=2,4, \ldots)$, whereas solving the Wronskian of the two even solutions will give the set $\mu_{1 n}(\lambda),(n=1,3, \ldots)$. The Wronskian functions, $F^{e v e n}(\mu, \lambda)$ and $F^{o d d}(\mu, \lambda)$, are defined as in Eqn. 2.47 and Eqn. 2.73. Fig 2.13 shows the Wronskian functions plotted as functions of $\mu$.

As shown in Fig. 2.13, the zeros of the Wronskian functions give all physically allowed separation parameters. For $m=1, F^{e v e n}(\mu, \lambda)$ determines $\mu_{11}, \mu_{13}, \mu_{15}$, which are approximately $-1.795305,-11.534818,-29.513713$, when $\lambda=1$. In the odd case $F^{o d d}(\mu, \lambda)$ determines $\mu_{12}, \mu_{14}$, which are approximately $-5.567527,-19.520683$, when $\lambda=1$. The values of the separation parameters are in agreement with those of [30,31]. (The separation parameter in $[30,31]$ is -1 times our separation parameter.) 


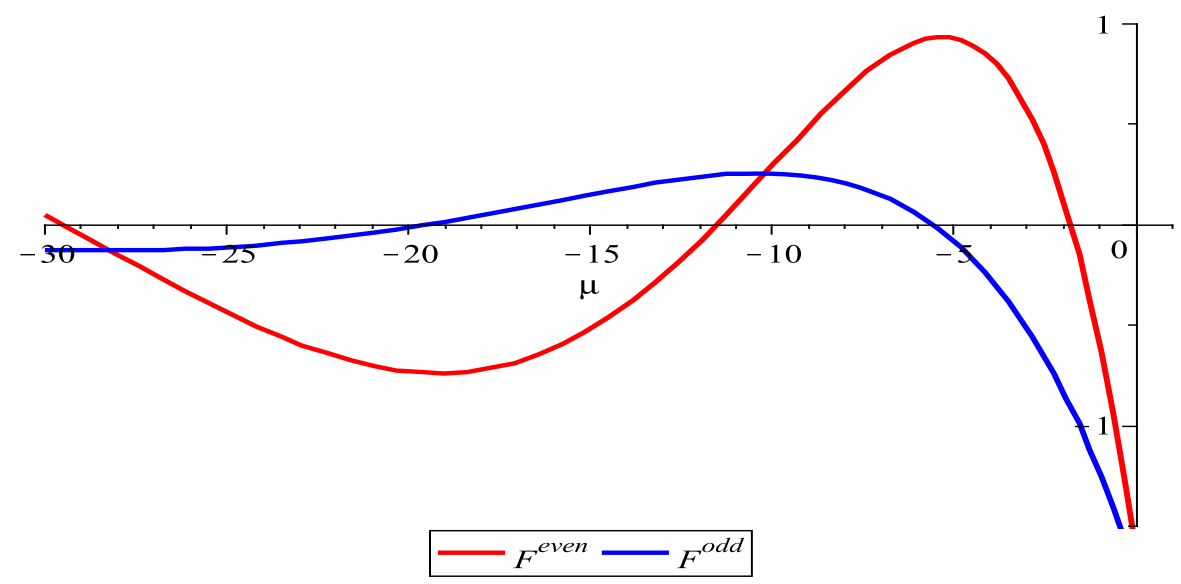

Figure 2.13: The Wronskian functions, $F^{e v e n}(\mu, \lambda)$ and $F^{o d d}(\mu, \lambda)$, plotted for $\lambda=1$, in the case where $m=1$. The zeros of both functions give the physically allowed values $\mu_{1 n}(\lambda)$. The first few zeros of $F^{e v e n}(\mu, \lambda)$ correspond to $\mu_{11}, \mu_{13}, \mu_{15}$, whereas the zeros of $F^{o d d}(\mu, \lambda)$ correspond to $\mu_{12}, \mu_{14}$.

Now that the $\mu_{m n}(\lambda)$ relation has been found, for arbitrary $m$, Eqn. 2.66 may be used to derive the $R(\lambda)$ dependency and determine the energy of $\mathrm{H}_{2}^{+}$in excited states. Bates et al. [39] have obtained the total energy of $\mathrm{H}_{2}^{+}$ for the $m=1$ gerade and ungerade states, which are shown in Fig. 2.14. (The values for $\mu_{m n}(\lambda)$ used by Bates et al. are those based on the series expansions.)

The ${ }^{2} \Pi_{u}$ energy curve shows a shallow minimum; however, the minimum energy is above the energy of a free proton and a hydrogen atom. The ${ }^{2} \Pi_{u}$ state is, therefore, a metastable state with respect to a free proton and a hydrogen atom. The ${ }^{2} \Pi_{g}$ state is repulsive for all internuclear separations and corresponds to an anti-bonding state. It is only the ${ }^{2} \Sigma_{g}^{+}$state which has a minimum below the energy of a free proton and a hydrogen atom. All other excited states, with arbitrary $m$, either have a minimum above this energy, or are repulsive for all internuclear separations. Thus the most important state is the bonding ground state. 


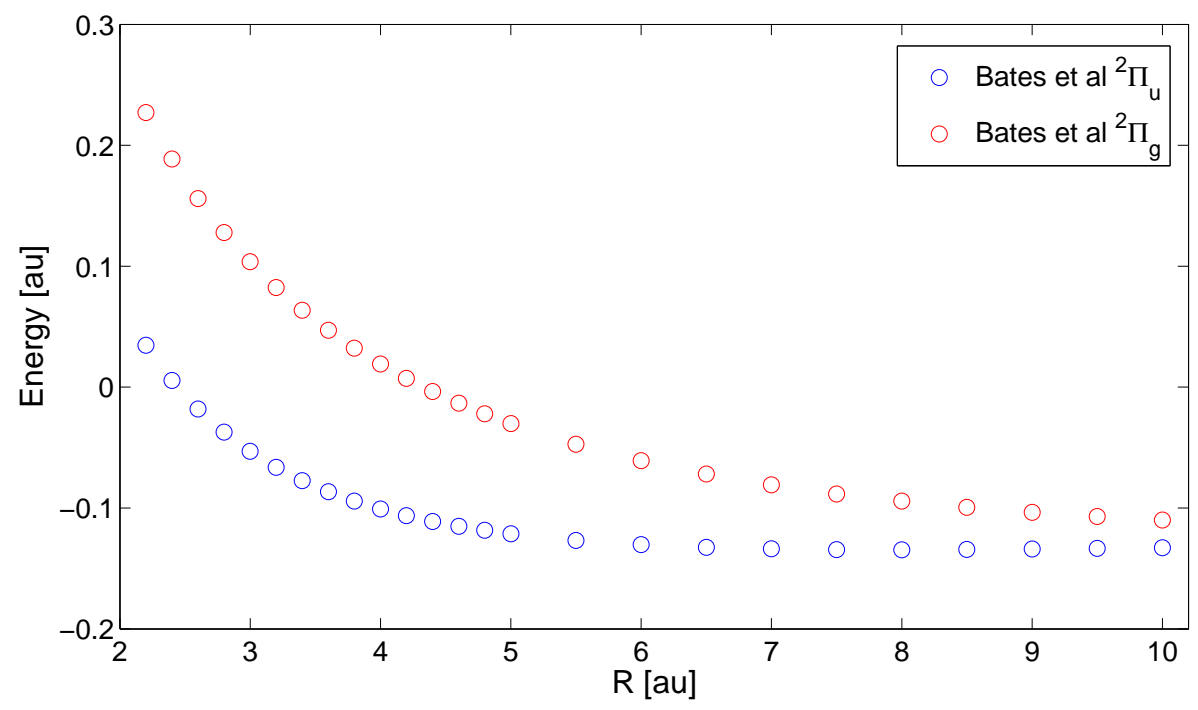

Figure 2.14: The total energy of $\mathrm{H}_{2}^{+}$in two excited states. The repulsive state which has no minimum (red curve) is a ${ }^{2} \Pi_{g}$ state, while the state with a shallow minimum (blue curve) is a ${ }^{2} \Pi_{u}$ state. (In the notation of [39] the ${ }^{2} \Pi_{g}$ state is labelled $3 d \pi_{g}$, while the ${ }^{2} \Pi_{u}$ state is labelled $2 p \pi_{u}$.)

In this Chapter the Wronskian method has been shown to give exact determination of the separation parameter $\mu_{m n}(\lambda)$ in the angular equation. We have shown that, in either the even or odd case, all physically allowed separation parameters may be obtained by the roots of a single Wronskian function. It was not possible to extend the Wronskian method to the radial case, however, this is an area for further research.

\subsection{An invariant of the motion}

The classical Laplace-Runge-Lenz vector is well-known in classical mechanics [40]. It is an invariant of the motion (it has zero time derivative) and its formulation allows the description of the path of a classical body under the influence of a centrally symmetric field. Pauli [41] was also able to solve the hydrogen atom energy spectrum by considering a similar invariant of the motion. 
Erikson and Hill [42] showed that

$$
\Omega=\frac{1}{2 \hbar^{2}}\left(\mathbf{L}_{\mathbf{1}} \cdot \mathbf{L}_{\mathbf{2}}+\mathbf{L}_{\mathbf{2}} \cdot \mathbf{L}_{\mathbf{1}}\right)+2 a \frac{m_{e} e^{2}}{\hbar^{2}}\left(Z_{1} \cos \theta_{1}+Z_{2} \cos \theta_{2}\right),
$$

is an invariant for the generalized one-electron binuclear ion, with nuclear charges $Z_{1}$ and $Z_{2}$. (This generalized problem is also solvable by separation of variables in elliptic coordinates.) In this expression $a=R / 2$ where $R$ is the nuclear separation. $\mathbf{L}_{\mathbf{1}}$ and $\mathbf{L}_{\mathbf{2}}$ are the orbital angular momenta of the electron about the nuclei 1 and 2 .

The geometry of this configuration is shown in Fig. 2.1 with the two nuclei located on the $z$ axis. However, the origin has been shifted to midway between the nuclei. The electron is a distance $\rho$ from the nuclear axis and a distance $z$ from the origin (along the $z$ axis). Therefore, $\cos \left(\theta_{1}\right)=(a+z) / r_{1}$ and $\cos \left(\theta_{2}\right)=(a-z) / r_{2}$.

If we denote the electron orbital angular momentum about the origin (at the midpoint between the nuclei) by $\mathbf{L}$, where $L_{x}=(\mathbf{r} \times \mathbf{p})_{x}=-i \hbar\left(y \partial_{z}-z \partial_{y}\right)$, then the following expressions are found

$$
\begin{aligned}
& \begin{cases}L_{1 x}=L_{x}+i \hbar a \partial_{y}, & L_{2 x}=L_{x}-i \hbar a \partial_{y} . \\
L_{1 y}=L_{y}-i \hbar a \partial_{x}, & L_{2 y}=L_{y}+i \hbar a \partial_{x} . \\
L_{1 z}=L_{z}, & L_{2 z}=L_{z} .\end{cases} \\
L^{2}= & \frac{1}{2}\left(\mathbf{L}_{\mathbf{1}} \cdot \mathbf{L}_{\mathbf{2}}+\mathbf{L}_{\mathbf{2}} \cdot \mathbf{L}_{\mathbf{1}}\right)-\hbar^{2} a^{2}\left(\partial_{x}^{2}+\partial_{y}^{2}\right), \\
= & \hbar^{2}\left\{-x^{2}\left(\partial_{y}^{2}+\partial_{z}^{2}\right)-y^{2}\left(\partial_{z}^{2}+\partial_{x}^{2}\right)-z^{2}\left(\partial_{x}^{2}+\partial_{y}^{2}\right)\right. \\
+ & \left.2\left[y z \partial_{y} \partial_{z}+z x \partial_{z} \partial_{x}+x y \partial_{x} \partial_{y}+x \partial_{x}+y \partial_{y}+z \partial_{z}\right]\right\} .
\end{aligned}
$$

The electronic Hamiltonian for the generalized one-electron binuclear ion (in $[\mathrm{au}]$ ) is

$$
H_{e}=-\frac{1}{2}\left(\partial_{x}^{2}+\partial_{y}^{2}+\partial_{z}^{2}\right)-\frac{Z_{1}}{r_{1}}-\frac{Z_{2}}{r_{2}} .
$$

Using the expressions for the electronic Hamiltonian and $\Omega$ given in Eqn. 2.79 and Eqn. 2.76, the following commutation relations can be deduced

$$
\begin{aligned}
{\left[L_{z}, H_{e}\right] } & =0 \\
{\left[\Omega, L_{z}\right] } & =0 \\
{\left[\Omega, H_{e}\right] } & =0 .
\end{aligned}
$$


Since $\Omega$ commutes with $H_{e}$ there exist simultaneous eigenstates of $\Omega$ and $H_{e}$. Thus, the eigenvalue equation $\Omega \psi=\omega \psi$ for $\Omega$ will correspond to the eigenvalue equation $H_{e} \psi=E \psi$ for $H_{e}$, with $\psi$ an eigenfunction of both operators.

In bipolar coordinates (using [au]) the operator $\Omega$ becomes

$$
\begin{aligned}
\Omega & =\frac{R^{2}+r_{1}^{2}-r_{2}^{2}}{2 r_{1}}\left(\partial_{r_{1}}+Z_{1}\right)+\frac{R^{2}-r_{1}^{2}+r_{2}^{2}}{2 r_{2}}\left(\partial_{r_{2}}+Z_{2}\right) \\
& +\frac{R^{2} \rho^{2}}{r_{1} r_{2}} \partial_{r_{1}} \partial_{r_{2}}+\frac{R^{2}-r_{1}^{2}-r_{2}^{2}}{2 \rho^{2}} \partial_{\phi}^{2},
\end{aligned}
$$

where $\rho$ is given in Eqn. 2.11. Using elliptic coordinates, as in section 2.2, with $\rho^{2}=(R / 2)^{2}\left(\xi^{2}-1\right)\left(1-\eta^{2}\right)$ and $z=(R / 2) \xi \eta$, the invariant $\Omega$, omitting azimuthal dependence, is found to be

$$
\begin{aligned}
& \Omega=\frac{1}{\xi^{2}-\eta^{2}}\left\{\left(1-\eta^{2}\right) \partial_{\xi}\left(\xi^{2}-1\right) \partial_{\xi}-\left(\xi^{2}-1\right) \partial_{\eta}\left(1-\eta^{2}\right) \partial_{\eta}\right. \\
& \left.+R\left[\left(Z_{1}+Z_{2}\right) \xi\left(1-\eta^{2}\right)+\left(Z_{1}-Z_{2}\right) \eta\left(\xi^{2}-1\right)\right]\right\} .
\end{aligned}
$$

The eigenvalue equation $\Omega \psi=\omega \psi$ reads

$$
\begin{array}{r}
\left\{\frac{1}{\xi^{2}-1} \partial_{\xi}\left(\xi^{2}-1\right) \partial_{\xi}-\frac{1}{1-\eta^{2}} \partial_{\eta}\left(1-\eta^{2}\right) \partial_{\eta}\right. \\
\left.+R\left(Z_{1}+Z_{2}\right) \frac{\xi}{\xi^{2}-1}+R\left(Z_{1}-Z_{2}\right) \frac{\eta}{1-\eta^{2}}-\frac{\omega}{\xi^{2}-1}-\frac{\omega}{1-\eta^{2}}\right\} \psi=0 .
\end{array}
$$

The eigenvalue equation derived in Eqn. 2.85 is separable in elliptic coordinates. As in section 2.2, we let $\psi(\xi, \eta)=X(\xi) Y(\eta)$. The eigenvalue equation given in Eqn. 2.85 can be rewritten as the following coupled ordinary differential equations for $X(\xi)$ and $Y(\eta)$ :

$$
\begin{aligned}
& \frac{d}{d \xi}\left\{\left(\xi^{2}-1\right) \frac{d X}{d \xi}\right\}+\left[R\left(Z_{1}+Z_{2}\right) \xi-\omega\right] X-\left(\xi^{2}-1\right) \nu X=0 \\
& \frac{d}{d \eta}\left\{\left(1-\eta^{2}\right) \frac{d Y}{d \eta}\right\}-\left[R\left(Z_{1}-Z_{2}\right) \eta-\omega\right] Y-\left(1-\eta^{2}\right) \nu Y=0
\end{aligned}
$$

where $\nu$ is the separation of variables constant.

The one-electron binuclear ion has a Hamiltonian (in [au]) given by

$$
H_{e}=-\frac{1}{2} \nabla^{2}-\frac{Z_{1}}{r_{1}}-\frac{Z_{2}}{r_{2}}
$$


where the Laplacian in elliptic coordinates is given in Eqn. 2.16. Setting $\psi=X(\xi) Y(\eta)$, and omitting the azimuthal variation (that is in the case $m=0$ ), we find that the electronic energy eigenvalue equation $H_{e} \psi=E \psi$, with $\lambda=-E R^{2} / 2$ and separation of variables constant $\mu$, gives

$$
\begin{gathered}
\frac{d}{d \xi}\left\{\left(\xi^{2}-1\right) \frac{d X}{d \xi}\right\}+\left[R\left(Z_{1}+Z_{2}\right) \xi\right] X+\left(-\lambda \xi^{2}+\mu\right) X=0, \\
\frac{d}{d \eta}\left\{\left(1-\eta^{2}\right) \frac{d Y}{d \eta}\right\}-\left[R\left(Z_{1}-Z_{2}\right) \eta\right] Y+\left(\lambda \eta^{2}-\mu\right) Y=0 .
\end{gathered}
$$

Comparison of the equations for $X(\xi)$ in Eqn. 2.86 and Eqn. 2.89, and for $Y(\eta)$ in Eqn. 2.87 and Eqn. 2.90, shows that they are equivalent if the following two conditions are met by the separation of variables constants

$$
\begin{aligned}
\nu & =\lambda, \\
\omega & =\lambda-\mu .
\end{aligned}
$$

Thus the separation of variables of $\Omega$, in the case $m=0$, leads to exactly the same coupled differential equations that arise on the separation of variables in Schrödinger's equation. As has been pointed out by Erikson and Hill [42], separation of the operator $\Omega$ is possible only in the case $m=0$. It seems the operator $\Omega$ will not allow the solution to the problem, since in this case the projections of the operator $\Omega$ and $H_{e}$ onto the $m=0$ subspace give the same eigenvalue equations. 


\section{Chapter 3}

\section{Scalar and Electromagnetic beams}

This Chapter focuses on the study of free space scalar and electromagnetic beams represented by solutions of Helmholtz's equation. In the case of scalar beams, Helmholtz's equation is separable in oblate spheroidal coordinates. The resulting angular equation is the same as the angular equation that arises in the study of $\mathrm{H}_{2}^{+}$. This connection allows the physical values of the separation parameter for scalar beams to be given by the zeros of the Wronskian of a pair of solutions to the angular equation. We shall derive this relation explicitly and show that, in both the $\mathrm{H}_{2}^{+}$problem and the scalar beam problem, the solution to the angular equation is a confluent Heun function. Following this, exact beam wave functions, initially in terms of confluent Heun functions, and subsequently in terms of oblate spheroidal wave functions, shall be investigated. For the oblate spheroidal wave functions to represent nonparaxial scalar beams the angular function $S_{m n}(\beta, \eta)$ requires odd $n-m$. However, they do not represent physical scalar beams, as this subset leads to wave functions with a discontinuous $z$ derivative, in the entire focal plane $z=0$. The properties of these exact beam wave functions shall be compared to the exact spherical Bessel beams and approximate paraxial Gaussian beam. A discussion is also presented of the generalized Bessel beams and the Bateman solution of the wave equation, which express the beam solutions as certain integrals. 


\subsection{Introduction}

The description of all electromagnetic phenomena is governed by Maxwell's equations plus the Lorentz force law. In SI units the Maxwell equations read

(iii) $\quad \nabla \times \mathbf{E}+\frac{\partial \mathbf{B}}{\partial t}=0 \quad$ (Faraday's law),

(iv) $\nabla \times \mathbf{B}-\mu_{0} \epsilon_{0} \frac{\partial \mathbf{E}}{\partial t}=\mu_{0} \mathbf{J}$ (Ampère - Maxwell law).

By applying the curl operator to the curl equations, and using the identity $\nabla \times(\nabla \times \mathbf{A})=\nabla(\nabla \cdot \mathbf{A})-\nabla^{2} \mathbf{A}$, we obtain the wave equation for the electric and magnetic fields. In free space, with no charge or current sources, these become

$$
\nabla^{2} \mathbf{E}=\frac{1}{c^{2}} \frac{\partial^{2} \mathbf{E}}{\partial t^{2}}, \quad \nabla^{2} \mathbf{B}=\frac{1}{c^{2}} \frac{\partial^{2} \mathbf{B}}{\partial t^{2}},
$$

where $c=1 / \sqrt{\mu_{0} \epsilon_{0}}$ is the speed of light.

Assuming the electric and magnetic fields have harmonic time dependence $\exp (-i \omega t)$, where $\omega=c k$, we obtain Helmholtz's equation for the scalar amplitude $\psi(\mathbf{r})$

$$
\left(\nabla^{2}+k^{2}\right) \psi(\mathbf{r})=0,
$$

where $\psi(\mathbf{r})$ is any one of the components of $\mathbf{E}(\mathbf{r})$ or $\mathbf{B}(\mathbf{r})$. Solving Eqn. 3.3, subject to Eqns. 3.1, for the electric and magnetic fields directly is often a difficult problem. Therefore, it is often advantageous to use the potential formulation of electrodynamics.

A continuous electromagnetic beam, with angular frequency $\omega=c k$, has electric and magnetic fields $\mathbf{E}(\mathbf{r}, t)$ and $\mathbf{B}(\mathbf{r}, t)$ which can be found from the complex magnetic vector potential $\mathbf{A}(\mathbf{r}, t)$ and the scalar potential $\Phi(\mathbf{r}, t)$ as follows:

$$
\begin{aligned}
& \mathbf{B}=\nabla \times \mathbf{A}, \\
& \mathbf{E}=-\nabla \Phi-\frac{\partial \mathbf{A}}{\partial t} .
\end{aligned}
$$


These two equations satisfy, identically, the two homogeneous Maxwell equations. The behaviour of the potentials is consequently determined by the two inhomogeneous Maxwell equations. In free space the two inhomogeneous equations of Eqns. 3.1 can be written in terms of the potentials as

$$
\begin{aligned}
\nabla^{2} \Phi+\frac{\partial}{\partial t}(\nabla \cdot \mathbf{A}) & =0 \\
\nabla^{2} \mathbf{A}-\frac{1}{c^{2}} \frac{\partial^{2} \mathbf{A}}{\partial t^{2}}-\nabla\left(\nabla \cdot \mathbf{A}+\frac{1}{c^{2}} \frac{\partial \Phi}{\partial t}\right) & =0 .
\end{aligned}
$$

The magnetic vector potential is arbitrary to the extent that we may add the gradient of some scalar $(\Lambda(\mathbf{r}, t))$, whereas the scalar potential is arbitrary in that we may subtract the time derivative of the same scalar quantity $(\Lambda(\mathbf{r}, t))$. This freedom allows us to impose a suitable gauge transformation which relates the potentials $\mathbf{A}$ and $\Phi$ to each other. (The invariance of the fields under such gauge transformations is known as gauge invariance.) One of the most commonly used gauges is the Lorenz gauge because it treats $\mathbf{A}$ and $\Phi$ on an equal level, and also because of its suitability in relativistic electrodynamics. The Lorenz gauge is defined as

$$
\nabla \cdot \mathbf{A}+\frac{1}{c^{2}} \frac{\partial \Phi}{\partial t}=0
$$

The use of the Lorenz gauge in Eqn. 3.6 and Eqn. 3.7 uncouples the two equations and leads to two separate wave equations with terms involving only A or $\Phi$ respectively:

$$
\begin{aligned}
\nabla^{2} \Phi-\frac{1}{c^{2}} \frac{\partial^{2} \Phi}{\partial t^{2}} & =0 \\
\nabla^{2} \mathbf{A}-\frac{1}{c^{2}} \frac{\partial^{2} \mathbf{A}}{\partial t^{2}} & =0 .
\end{aligned}
$$

If we assume harmonic time dependence $\exp (-i \omega t)$, where $\omega=c k$, for both the potentials $\mathbf{A}$ and $\Phi$, then Eqn. 3.9 and Eqn. 3.10 reduce to the Helmholtz equation Eqn. 3.3, but now the interpretation of $\psi(\mathbf{r})$ is that it is associated with one of the potentials. Thus in free space, $\Phi$ and all three components of A satisfy Eqn. 3.3, which allows both the electric and magnetic fields to be subsequently derived. 
As has already been mentioned, this formulation of electrodynamics is advantageous in that deriving the fields from the potentials is easier than solving for the fields directly. Section 3.7 shall investigate the construction of electric and magnetic fields from the solutions of the Helmholtz equation for the potentials. Chapter 6, section 5 of Jackson [43], and Chapter 10 of Griffiths [44] give further details on the potential formulation of electrodynamics.

In the quantum particle beam case we shall assume that the probability amplitude $\Psi(\mathbf{r}, t)$ represents a coherent beam of spinless, non-interacting particles of mass $m$, each with energy $\hbar^{2} k^{2} / 2 m$, where $k$ is the wavenumber. The time evolution of the particle beam is governed by the Schrödinger equation $\hat{H} \Psi=i \hbar \partial \Psi / \partial t$, where $\hat{H}$ is an effective Hamiltonian for an atom of mass $m$ in an external potential $V(\mathbf{r})$ :

$$
\hat{H}=-\frac{\hbar^{2}}{2 m} \nabla^{2}+V(\mathbf{r})
$$

From the Schrödinger equation the well-known continuity equation follows

$$
\frac{\partial|\Psi|^{2}}{\partial t}+\nabla \cdot\left\{\frac{\hbar}{m} \operatorname{Im}\left(\Psi^{*} \nabla \Psi\right)\right\}=0,
$$

where the term in the parentheses is known as the probability current density J. The continuity equation physically represents the conservation of probability. By the divergence theorem the integral of $\mathbf{J}$ over a surface $S$ is equal to the decrease in probability of finding the particle in the (fixed) volume $V$, bounded by $S$,

$$
\oint_{S} \mathbf{J} \cdot d \mathbf{a}=-\frac{d}{d t} \int_{V}|\Psi|^{2} d^{3} \mathbf{r} .
$$

In the case of 'steady beams', i.e. a particle beam in an energy eigenstate with time evolution given by $\Psi(\mathbf{r}, t)=\psi(\mathbf{r}) \exp (-i \omega t)$, where $\omega=\hbar k^{2} / 2 m$, Schrödinger's time-independent equation in free space is given by $\left(\nabla^{2}+\right.$ $\left.k^{2}\right) \psi(\mathbf{r})=0$. In order to describe particle beams we must also solve Eqn. 3.3; the interpretation of $\psi(\mathbf{r})$ now being a probability amplitude for the particle beam, which satisfies the continuity equation Eqn. 3.12. 
Lekner [45] discusses exact particle beams in terms of spherical Bessel beams, to be defined later, and discusses some of their properties such as their momentum content per unit length. There are also experimental investigations of focusing and diffracting Helium atom beams referenced in [45]. The next section shall investigate exact solutions of the Helmholtz equation by using oblate spheroidal coordinates.

\subsection{Oblate spheroidal coordinates}

In section 2.2 the (electronic) Schrödinger equation for the $\mathrm{H}_{2}^{+}$problem was shown to be separable in prolate spheroidal coordinates. For the scalar beam problem we use oblate spheroidal coordinates, in which

$$
\begin{gathered}
\rho=b \sqrt{\xi^{2}+1} \sqrt{1-\eta^{2}}, \quad z=b \xi \eta, \quad \phi=\phi \\
0 \leq \eta \leq 1, \quad-\infty<\xi<\infty, \quad 0 \leq \phi \leq 2 \pi .
\end{gathered}
$$

The oblate spheroidal coordinate system, shown in Fig. 3.1, is formed by rotating the two-dimensional elliptic coordinate system, consisting of confocal ellipses and hyperbolas, about the minor axis of the ellipse. The interfocal distance is $2 b$, and it is customary to make the $z$ axis the axis of revolution. (The prolate spheroidal coordinate system is obtained by rotating about the major axis.) As defined, the oblate spheroidal coordinate system is an orthogonal curvilinear coordinate system.

The closed form expressions for the ellipsoids, or hyperboloids, of the oblate spheroidal coordinate system can be obtained by eliminating one of the variables in Eqns. 3.14. For example, eliminating $\eta$ from Eqns. 3.14, we get the ellipsoid

$$
\frac{\rho^{2}}{b^{2}\left(\xi_{0}^{2}+1\right)}+\frac{z^{2}}{b^{2} \xi_{0}^{2}}=1 .
$$

Likewise, by setting $\eta=\eta_{0}$ and eliminating $\xi$ from Eqns. 3.14, we get the hyperboloid

$$
\frac{\rho^{2}}{b^{2}\left(1-\eta_{0}^{2}\right)}-\frac{z^{2}}{b^{2} \eta_{0}^{2}}=1 .
$$




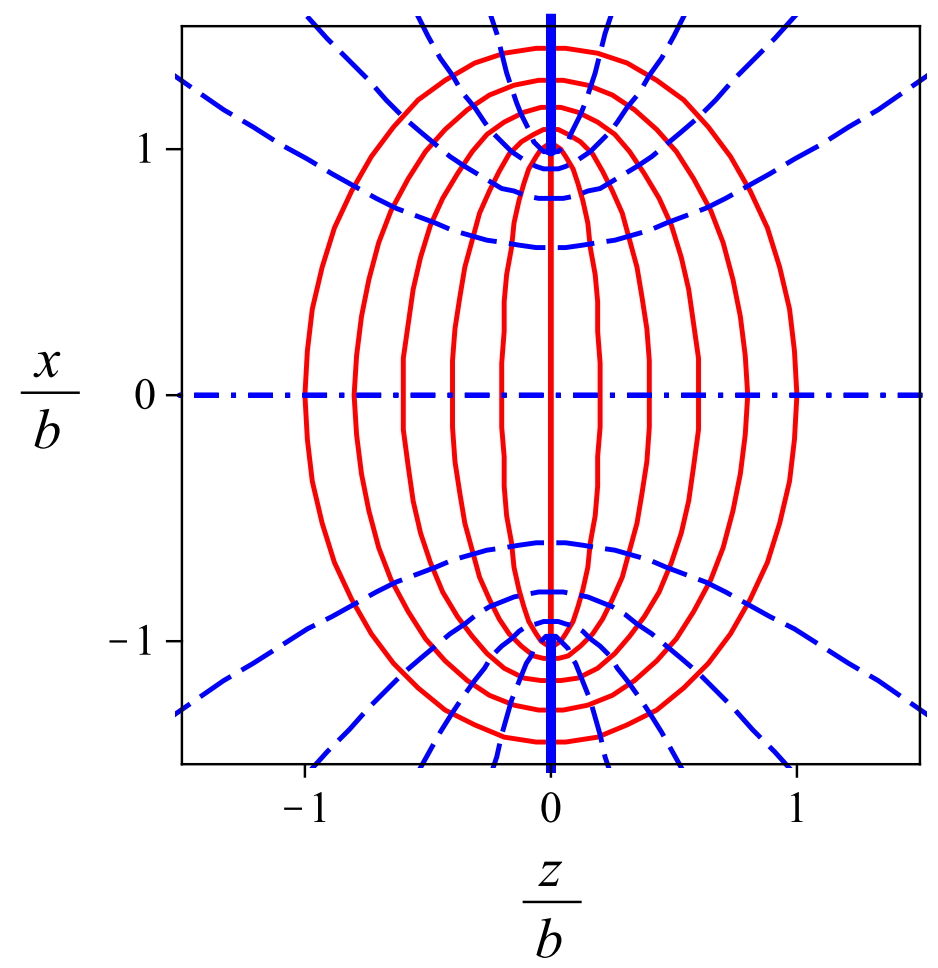

Figure 3.1: The oblate spheroidal coordinate system. The confocal ellipsoids (lines) are surfaces of constant $\xi$. The central ellipsoid (the disk $z=0, \rho \leq b$ ) is at $\xi=0$, with $\xi$ increasing or decreasing by 0.2 in each of the outer half-ellipsoids. The confocal hyperboloids (dashed) are surfaces of constant $\eta$. In the focal plane $z=0$ the region outside the disk $\rho \geq b$ is shown by the solid line $\eta=0$. The $z$ axis is given by $\eta=1$. The hyperboloids increase in $\eta$ by 0.2 from the $\eta=0$ central hyperboloid.

In the oblate spheroidal coordinate system a surface of constant $\left|\xi_{0}\right|(>$ $0)$ is a flattened ellipsoid of revolution with major axis of length $2 b\left(\xi_{0}^{2}+1\right)^{1 / 2}$ and minor axis of length $2 b\left|\xi_{0}\right|$. The surface $\xi_{0}=0$ is a circular disk of radius $b$ which lies in the $x, y$ plane and is centred at the origin. The surface $\eta=$ constant $(<1)$ is a hyperboloid of revolution of one sheet, with an asymptotic cone whose generating line passes through the origin, and is inclined at an angle $\theta=\cos ^{-1} \eta$ to the $z$ axis. The surface $\phi=$ constant is the plane through the $z$ axis making angle $\phi$ with the $x, z$ plane. 
In relation to scalar beams the axis along which the beam propagates, known as the beam axis, is taken to be the $z$ axis, which in the oblate spheroidal coordinate system corresponds to $\eta=1$ with $\xi$ ranging from -infinity to infinity. The focal plane of the beam is a little more complicated: $z=0$ corresponds to $\eta=0$ for $\rho \geq b$, while for $\rho \leq b$ it corresponds to $\xi=0$.

The parameter $b$ in Eqns. 3.14 determines the length scale of the coordinate system, and also properties of the scalar beam. In the limit of large $k b$ the well-known Gaussian beam is a good approximation to an exact scalar beam solution of the Helmholtz equation. The Gaussian beam is [46]

$$
\psi_{G}=\frac{b}{b+i z} \exp \left[i k z-\frac{k \rho^{2}}{2(b+i z)}\right] .
$$

The length of the focal region of the beam is given by $b$. The beam waist (at $z=0)$ is $W_{0}=(2 b / k)^{1 / 2}$, since there $\left|\psi_{G}\right|=\exp \left(-\rho^{2} / W_{0}^{2}\right)$. Physically the beam waist represents the minimum width of the beam in the focal plane $(z=0)$, whereas along the beam axis $(\rho=0, z>0)$ the width of the beam increases with distance from the focal plane corresponding to angular spread in the wavefronts.

Throughout this Chapter we shall use the parameter $\beta=k b$ in order to describe the type of beam. For example, when $\beta$ is large the beam waist is many wavelengths wide and the focal region is many wavelengths long. In the large $\beta$ (paraxial) case the angular divergence of the beam from the $z$ axis, given by the angle $\alpha$, is related to $\beta$ by $\tan \alpha=(2 / \beta)^{1 / 2}$ [47]. Thus large $\beta$ corresponds to a weakly focused beam whose angular divergence from the beam axis is small.

In contrast, a small value of $\beta$ corresponds to a tightly focused beam whose width increases along the beam axis. A beam with a small beam waist (small $\beta$ ) is tightly squeezed in the focal region and consequently spreads in width along the beam axis. Further discussion of the properties of beams may be found in Chapter 3 of [46]. We shall now seek exact solutions of the Helmholtz equation for scalar beams, by utilizing the separability of the Helmholtz equation in oblate spheroidal coordinates. 


\subsection{Angular and radial equations}

The Laplacian in oblate spheroidal coordinates, omitting azimuthal variation, is

$$
\nabla^{2}=\frac{1}{b^{2}\left(\xi^{2}+\eta^{2}\right)}\left\{\partial_{\xi}\left(\xi^{2}+1\right) \partial_{\xi}+\partial_{\eta}\left(1-\eta^{2}\right) \partial_{\eta}\right\} .
$$

The Helmholtz equation is thus

$$
\left\{\partial_{\xi}\left(\xi^{2}+1\right) \partial_{\xi}+\partial_{\eta}\left(1-\eta^{2}\right) \partial_{\eta}+\beta^{2}\left(\xi^{2}+\eta^{2}\right)\right\} \psi(\xi, \eta)=0 .
$$

Setting $\psi(\xi, \eta)=X(\xi) Y(\eta)$ in Eqn. 3.19 we obtain the following angular and radial equations, with $\mu$ as the separation of variables parameter

$$
\begin{aligned}
& \frac{d}{d \eta}\left\{\left(1-\eta^{2}\right) \frac{d Y}{d \eta}\right\}+\left(\beta^{2} \eta^{2}-\mu\right) Y=0, \\
& \frac{d}{d \xi}\left\{\left(\xi^{2}+1\right) \frac{d X}{d \xi}\right\}+\left(\beta^{2} \xi^{2}+\mu\right) X=0 .
\end{aligned}
$$

The parameter $\mu$ takes only certain physical values dependent on $\beta$. In the case where there is no azimuthal variation the allowed values of the separation of variables parameter are denoted by $\mu_{0 n}(\beta), n=0,1,2, \ldots$. If we had included azimuthal variation in the Helmholtz equation then we would obtain eigenfunctions $\exp ( \pm i m \phi)$ in the wave function, which would lead to a separation of variables parameter $\mu_{m n}(\beta)$, i.e. with $m$ dependence, see Chapter 3 of [31]. In this case, however, we may neglect the $m$ dependence since it only gives the scalar beam a certain amount of angular momentum.

By comparison with the angular equation Eqn. 2.39, we have that the angular equation is exactly the same. Thus, as mentioned at the beginning of this Chapter, we have this remarkable connection between the $\mathrm{H}_{2}^{+}$problem in prolate spheroidal coordinates and the scalar beam problem in oblate spheroidal coordinates. (Oblate rather than prolate because the energy of $\mathrm{H}_{2}^{+}$is negative.) Both problems have the same angular equation, with $\lambda=\beta^{2}$. As the solution to the angular equation for which $Y(\eta)$ is finite at $\eta^{2}=1$ determines $\mu_{m n}(\lambda)$ or $\mu_{m n}(\beta)$, the angular problem for $\mathrm{H}_{2}^{+}$and for beams is equivalent. This relation further elucidates a reason why the $\mathrm{H}_{2}^{+}$problem is of such importance. Solving this problem would allow the solution of (separable) scalar beams, in oblate spheroidal coordinates. 
The angular equation has two linearly independent solutions, where one is even and the other is odd. There is a pair of even solutions, and a pair of odd solutions. One of each pair is regular at the ordinary point $\eta=0$, and the other solution is regular at the regular points $\eta^{2}=1$, for arbitrary $\mu$. These types of solutions have already been discussed in Chapter 2.

The two even solutions, one which is regular at $\eta=0$ and the other regular at $\eta^{2}=1$, are confluent Heun functions and are given by

$$
\begin{aligned}
& Y_{0}^{\text {even }}(\eta)=\operatorname{HeunC}\left(0,-\frac{1}{2}, 0, \frac{-\beta^{2}}{4}, \frac{\mu+1}{4}, \eta^{2}\right) \\
& Y_{1}^{\text {even }}(\eta)=\operatorname{HeunC}\left(0,0,-\frac{1}{2}, \frac{\beta^{2}}{4}, \frac{\mu-\beta^{2}+1}{4}, 1-\eta^{2}\right) .
\end{aligned}
$$

The two odd angular functions, one which is regular at $\eta=0$ and the other regular at $\eta^{2}=1$, are given by

$$
\begin{aligned}
& Y_{0}^{\text {odd }}(\eta)=\eta \operatorname{HeunC}\left(0, \frac{1}{2}, 0, \frac{-\beta^{2}}{4}, \frac{\mu+1}{4}, \eta^{2}\right) \\
& Y_{1}^{\text {odd }}(\eta)=\eta \operatorname{HeunC}\left(0,0, \frac{1}{2}, \frac{\beta^{2}}{4}, \frac{\mu-\beta^{2}+1}{4}, 1-\eta^{2}\right) .
\end{aligned}
$$

The notation $\mu_{e}(\beta), \mu_{o}(\beta)$ shall be used in this Chapter for convenience since we shall need to distinguish between both even and odd solutions, and also even and odd separation parameters simultaneously. In this case $\mu_{e}(\beta)=\mu_{0 n}(\beta)$, where $n=0,2,4, \ldots$ is an arbitrary even number, and similarly $\mu_{o}(\beta)=\mu_{0 n}(\beta)$, where $n=1,3,5, \ldots$ is an arbitrary odd number. The Wronskian of two linearly dependent angular solutions can be used to obtain the allowed separation parameters $\mu_{e / o}(\beta)$; the calculation is that of Chapter 2 with $\lambda=\beta^{2}$. The Wronskian in the scalar beam case also gives a transcendental relation between $\mu_{e / o}(\beta)$ and $\beta$ whose solution is equivalent with those obtained via the infinite series representations of $\mu_{e / o}(\beta)$.

In the oblate spheroidal coordinate system the radial equation for scalar beams is, however, different from that derived for $\mathrm{H}_{2}^{+}$. There is no parameter $R(\beta)$ in this instance, and so we only need a single Wronskian to determine the solution completely. It should be noted that in this case the radial equation may be obtained from the angular equation by the substitution $\eta \rightarrow i \xi$. 
This symmetry will play an important role later when we derive integral relations between the angular and radial solutions.

Since we only require the separation parameter $\mu_{e / o}(\beta)$, we need a single even and a single odd radial confluent Heun solution. There is no need for the second even or odd radial confluent Heun solution, which is regular at $\xi= \pm i$. The even and odd radial confluent Heun solutions, which are regular at $\xi=0$, are given by

$$
\begin{aligned}
& X_{0}^{\text {even }}(\xi)=\operatorname{HeunC}\left(0,-\frac{1}{2}, 0, \frac{-\beta^{2}}{4}, \frac{\mu+1}{4},-\xi^{2}\right), \\
& X_{0}^{\text {odd }}(\xi)=\xi \operatorname{HeunC}\left(0, \frac{1}{2}, 0, \frac{-\beta^{2}}{4}, \frac{\mu+1}{4},-\xi^{2}\right) .
\end{aligned}
$$

Now that the separable solutions of the Helmholtz equation have been found all other solutions may be obtained, either in the product form $X(\xi) Y(\eta)$, or through an infinite sum of such products. We shall concentrate on representing exact beam wave functions through the former approach, but before investigating these solutions there is an important physical constraint we must impose on the beam solutions. A physical beam in free space is forward propagating. Therefore we must fix the beam solution's asymptotic form to have purely forward directional propagating wavefronts. The next section shall investigate the asymptotic form of the radial solutions presented here.

\subsection{Asymptotics of the radial solutions}

In order to construct physical scalar or electromagnetic beams, propagating in free space, we require that the solutions of Helmholtz's equation are forward propagating. To ensure the solutions derived satisfy this constraint we must examine their asymptotic behaviour. This section will rigourously derive the asymptotic form of the even and odd radial solutions. Following this analysis we are then able to form a linear combination of the two radial solutions which represent a forward propagating beam solution.

The radial equation as derived previously is given by

$$
\left(\xi^{2}+1\right) \frac{d^{2} X}{d \xi^{2}}+2 \xi \frac{d X}{d \xi}+\left(\beta^{2} \xi^{2}+\mu\right) X=0 .
$$


In the asymptotic limit that $\beta \xi \rightarrow \infty$ the radial equation becomes

$$
\xi^{2} \frac{d^{2} X}{d \xi^{2}}+2 \xi \frac{d X}{d \xi}+\beta^{2} \xi^{2} X=0
$$

which may be written as

$$
\frac{d^{2}}{d \xi^{2}}(\xi X)+\beta^{2} \xi X=0 .
$$

The general solution to Eqn. 3.30 is given by

$$
X(\xi)=c_{1}(\beta) \frac{\cos (\beta \xi)}{\beta \xi}+c_{2}(\beta) \frac{\sin (\beta \xi)}{\beta \xi} .
$$

This represents the general asymptotic form of the solutions to the radial equation for large $\beta \xi$. The coefficient functions $c_{1}(\beta)$ and $c_{2}(\beta)$ are functions of $\mu$ (and hence of $\beta$ ) and depending on the particular values these functions take we will obtain the asymptotic form of the even and odd radial confluent Heun solutions.

The coefficients in Eqn. 3.31 are, however, undetermined. This is because the analysis performed here has been a local one and as such cannot determine the coefficient functions for each individual radial solution. In order to ascertain the coefficient functions we must perform a global analysis of the radial solutions. Such analysis is usually performed through integral representations of the solutions, which is not possible in this case. Whereas solutions of the hypergeometric differential equation have well-known integral representations there are, at this stage, no non-trivial integral representations for confluent Heun functions. Only integral relations in terms of simpler confluent Heun functions are known at this stage. See [34], [35] for further details.

The differential equation satisfied by the spherical Bessel functions $j_{n}(\beta \xi)$ is given by

$$
\xi^{2} \frac{d^{2} j_{n}}{d \xi^{2}}+2 \xi \frac{d j_{n}}{d \xi}+\left[\beta^{2} \xi^{2}-n(n+1)\right] j_{n}=0 .
$$

The correspondence between Eqn. 3.29 and Eqn. 3.32 at large $\xi$ is clear, and several authors have considered expansions in terms of spherical Bessel functions $[31,48]$. 
Therefore, despite the non-existence of an integral representation of the radial confluent Heun solutions, we can still perform a global analysis via an expansion in terms of spherical Bessel functions. Assuming such an expansion to exist, we write the even and odd radial solutions as follows

$$
\begin{aligned}
X_{0}^{\text {even }}(\xi) & =\sum_{n=0,2,4, \ldots}^{\infty} a_{n}(\beta) j_{n}(\beta \xi), \\
X_{0}^{\text {odd }}(\xi) & =\sum_{n=1,3,5, \ldots}^{\infty} b_{n}(\beta) j_{n}(\beta \xi) .
\end{aligned}
$$

The spherical Bessel functions do have an integral representation, which is

$$
j_{n}(z)=(-i)^{n} \frac{1}{2} \int_{-1}^{1} \exp (i z \cos (\theta)) P_{n}(\cos (\theta)) d(\cos (\theta)),
$$

where $P_{n}(\cos (\theta))$ represent the Legendre polynomials of the first kind. The spherical Bessel functions have expansions about the origin given by

$$
j_{n}(z)=z^{n} \sum_{l=0}^{\infty} \frac{\left(-z^{2} / 2\right)^{l}}{l !(2 n+2 l+1) ! !} .
$$

The even and odd radial solutions may also be expanded about the origin as Taylor series expansions:

$$
\begin{aligned}
& X_{0}^{\text {even }}(\xi)=\sum_{n=0,2,4, \ldots}^{\infty} A_{n}(\beta) \xi^{n}, \quad|\xi|<1, \\
& X_{0}^{\text {odd }}(\xi)=\sum_{n=1,3,5, \ldots}^{\infty} B_{n}(\beta) \xi^{n}, \quad|\xi|<1 .
\end{aligned}
$$

The coefficients, $A_{n}(\beta)$ and $B_{n}(\beta)$, are obtained from the radial differential equation and satisfy a three term recurrence relation, namely

$$
\begin{aligned}
& (n+\alpha)(n+\alpha-1) C_{n}(\beta) \\
+ & {[(n+\alpha-2)(n+\alpha-3)+\mu+2(n+\alpha-2)] C_{n-2}(\beta) } \\
+ & \beta^{2} C_{n-4}(\beta)=0, \quad n=0,2,4, \ldots,
\end{aligned}
$$

where $\alpha=0$ for the even solution, $\alpha=1$ for the odd solution, and in the appropriate case $C_{n}(\beta)$ will be either $A_{n}(\beta)$ or $B_{n+1}(\beta)$. 
Note that the terms with negative indices are zero, and $A_{0}=B_{1}=1$. The recurrence relation also provides a method of deriving the transcendental relation between $\mu_{0 n}(\beta)$ and $\beta$. We shall compare the method with the results observed in the study of $\mathrm{H}_{2}^{+}$and show, explicitly, that the same $\mu_{0 n}(\beta)$ series representations are obtained.

The radial series solutions are convergent for $|\xi|<1$, which requires a $\mu_{0 n}(\beta)$ such that the coefficients, $A_{n}(\beta)$ or $B_{n}(\beta)$, tend to zero as $n$ tends to $\infty$. Setting $C_{n}(\beta)$ in Eqn. 3.39 equal to zero gives equations for $\mu_{0 n}(\beta)$ of degree $n / 2$. Thus the transcendental relation, in order that we have the correct $\mu_{0 n}(\beta)$, is that $\lim _{n \rightarrow \infty} C_{n}(\beta)=0$. Solving $C_{8}$ equal to zero, for example, we find that the series representations for the lowest $\mu_{0 n}(\beta)$ obtained are given by

$$
\begin{aligned}
& \mu_{00}(\beta)=\frac{1}{3} \beta^{2}+\frac{2}{135} \beta^{4}+\frac{4}{8505} \beta^{6}+O\left(\beta^{8}\right), \\
& \mu_{01}(\beta)=-2+\frac{3}{5} \beta^{2}+\frac{6}{875} \beta^{4}-\frac{4}{65625} \beta^{6}+O\left(\beta^{8}\right) .
\end{aligned}
$$

The condition of a convergent series solution imposes the requirement that the coefficient functions tend to zero; it also imposes the condition that $\mu$ tends to the correct $\mu_{0 n}(\beta)$ with the same transcendental form as was derived in the study of the eigenvalues of $\mathrm{H}_{2}^{+}$. The even series solution for $\mu_{00}(\beta)$ obtained here agrees with the one derived in section 2.3 (with $\lambda=\beta^{2}$ ), and the odd series solution for $\mu_{01}(\beta)$ agrees with the odd $\mu_{01}(\lambda)$ that was derived in section 2.7 .

The expansion of the spherical Bessel functions about the origin, along with the Taylor expansion of the confluent Heun functions about the origin, means that, in principle, we can obtain the coefficients $a_{n}(\beta)$ and $b_{n}(\beta)$.

Define the functions $U(\beta, \cos (\theta))$ and $V(\beta, \cos (\theta))$ as follows

$$
\begin{aligned}
\sum_{n=0,2,4, \ldots}^{\infty}(-i)^{n} a_{n}(\beta) P_{n}(\cos (\theta)) & \equiv U(\beta, \cos (\theta)), \\
\sum_{n=1,3,5, \ldots}^{\infty}(-i)^{n} b_{n}(\beta) P_{n}(\cos (\theta)) & \equiv i V(\beta, \cos (\theta)) .
\end{aligned}
$$


The radial solutions then have the following form

$$
\begin{aligned}
X_{0}^{\text {even }}(\xi) & =\frac{1}{2} \int_{-1}^{1} \exp (i \beta \xi \cos (\theta)) U(\beta, \cos (\theta)) d(\cos (\theta)), \\
X_{0}^{\text {odd }}(\xi) & =\frac{i}{2} \int_{-1}^{1} \exp (i \beta \xi \cos (\theta)) V(\beta, \cos (\theta)) d(\cos (\theta)) .
\end{aligned}
$$

Integration by parts of the expression in Eqn. 3.44 gives

$$
X_{0}^{e v e n}(\xi)=\frac{1}{2 i \beta \xi}(U(\beta, 1) \exp (i \beta \xi)-U(\beta,-1) \exp (-i \beta \xi))+O\left(\xi^{-2}\right) .
$$

The sum in $U$ is over even $n$, and $P_{n}(-1)=(-1)^{n} P_{n}(1)$; hence we rigourously have that the asymptotic form of the even radial solution is given by

$$
X_{0}^{\text {even }}(\xi)=U(\beta, 1) \frac{\sin (\beta \xi)}{\beta \xi}+O\left(\xi^{-2}\right), \quad \beta \xi \rightarrow \infty .
$$

Thus, we have derived that for the even radial confluent Heun solution, returning to the general asymptotic solution in Eqn. 3.31, $c_{2}(\beta)=U(\beta, 1)$ and $c_{1}(\beta)=0$. This is subject to the constraint that $\mu(\beta)=\mu_{e}(\beta)$, with the series representation for the lowest $\mu_{e}(\beta)$ given in Eqn. 3.40.

Using the same method, we find that the odd radial confluent Heun solution has the asymptotic form given by

$$
X_{0}^{\text {odd }}(\xi)=\frac{i}{2 i \beta \xi}(V(\beta, 1) \exp (i \beta \xi)-V(\beta,-1) \exp (-i \beta \xi))+O\left(\xi^{-2}\right) .
$$

Therefore, with $V(\beta,-1)=-V(\beta, 1)$, we rigourously have that the asymptotic form of the odd radial confluent Heun solution is

$$
X_{0}^{\text {odd }}(\xi)=V(\beta, 1) \frac{\cos (\beta \xi)}{\beta \xi}+O\left(\xi^{-2}\right), \quad \beta \xi \rightarrow \infty .
$$

For the odd radial confluent Heun solution we now have, in the general asymptotic solution Eqn. 3.31, $c_{1}(\beta)=V(\beta, 1)$ and $c_{2}(\beta)=0$. This is subject to the constraint that $\mu(\beta)=\mu_{o}(\beta)$, with the series representation for the lowest $\mu_{o}(\beta)$ given in Eqn. 3.41. 
This analysis has successfully derived the asymptotic form of the even and odd radial confluent Heun solutions. In order to construct physical beam solutions the next task would be to ensure that the asymptotic nature of the full solution to the radial equation is proportional to $e^{i \beta \xi} / \beta \xi$, i.e. a forward propagating scalar beam. There is, however, a subtlety to the asymptotic solutions derived here. The even radial solution is asymptotic to $U(\beta, 1) \sin (\beta \xi) / \beta \xi$, while the odd radial solution is asymptotic to $V(\beta, 1) \cos (\beta \xi) / \beta \xi$. In order that the spherical Bessel series expansions are convergent we have had to impose the condition that for the even radial solution $\mu(\beta)=\mu_{e}(\beta)$, whereas for the odd radial solution $\mu(\beta)=\mu_{o}(\beta)$. The two radial solutions have different separation parameters.

Finding what separation parameters $\mu(\beta)$ will make one of $c_{1}(\beta)$ or $c_{2}(\beta)$ equal to zero, given that the solution about the origin is known, is called the central two-point connection problem [34], [35]. Thus, through the spherical Bessel expansions, we have shown what form the separation parameters $\mu(\beta)$ must take for only a single asymptotic solution to dominate. In the odd case $\mu(\beta)=\mu_{o}(\beta)$, while in the even case $\mu(\beta)=\mu_{e}(\beta)$.

There is one way to interpret this result: instead of the simple $X(\xi) Y(\eta)$ product solution to Helmholtz's equation, we have a solution which is given by $\psi(\xi, \eta)=X^{\text {even }}(\xi) Y^{\text {even }}(\eta)+X^{\text {odd }}(\xi) Y^{\text {odd }}(\eta)$ in which we use an even $\mu_{e}(\beta)$ for the even product, and an odd $\mu_{o}(\beta)$ for the odd product. However, this solution would not produce a forward propagating beam for arbitrary $\eta$. It would be possible to construct a forward propagating scalar beam along the beam axis $(\eta=1)$, but then at any region of space off the beam axis there will be an introduction of (nonphysical) backward propagation. In other words, there will be a linear superposition of waves propagating to the right and propagating to the left representing the scalar beam solution.

Thus, using a single $\mu(\beta)$ separation parameter is apparently the most physical representation of a forward propagating scalar beam solution of Helmholtz's equation. In the following sections we shall investigate the scalar beams resulting from using two separation parameters $\mu_{e}(\beta)$ and $\mu_{o}(\beta)$, and then consider exact beam solutions which have a single separation parameter. 


\subsection{Integral relations for confluent Heun func- tions}

In order to derive analytic expressions for the asymptotic expansions of the radial solutions an integral representation, or integral relation, is required. It is the aim of this section to derive an integral relation for the two radial solutions in terms of the angular confluent Heun functions. This integral relation will give a complete description of the asymptotic nature of the two radial solutions, and in the process we shall derive the sums of the alternating series $U(\beta, 1)$ and $V(\beta, 1)$. The method employed is that used by Flammer, who obtains the same integral relations for the oblate spheroidal functions [31]. Before presenting the derivation an introduction to the theory of integral equations and integral relations is given.

A Fredholm integral equation of the first kind is of the form

$$
f(x)=\int_{a}^{b} K(x, t) \phi(t) d t .
$$

In this case $f(x)$ represents a known function, $\phi(t)$ is the function to be determined, and $K(x, t)$ is called the kernel function. The two limits are fixed, which is what determines the integral equation as one of the Fredholm type. If an unknown function is present both under the integral sign and outside it, then we have a Fredholm integral equation of the second kind. In the case of a variable limit of integration the integral equation becomes a Volterra integral equation of the first or second kind.

An integral relation for a solution of the confluent Heun equation (CHE) is one of the form

$$
w(z)=\int_{C} K(\phi(z, t)) v(t) d t
$$

where the functions $w(z)$ and $v(z)$ are specially chosen solutions of the same or different CHE, and $\phi(z, t)$ is a 'simpler' function. The contour of integration can be either a closed contour on a Riemannian surface of the integration variable or a contour with end points at singularities of the CHE. 
In an integral equation the unknown function is under the integral sign, whereas in an integral relation a function is expressed in terms of an integral of another function which solves a similar CHE. The function $K(\phi(z, t))$ has been shown to be a solution of confluent hypergeometric differential equations in certain cases and under additional assumptions the equations for $v(z)$ lead to certain Fredholm integral equations. There is an extensive treatment of this topic in [34], [35], and [49] which should be referred to for further details. We shall now give a derivation of an integral relation for both the even and odd radial confluent Heun solutions relevant for scalar beams.

Let $L_{\eta}$ be the linear, self-adjoint differential operator

$$
L_{\eta}=\frac{\partial}{\partial \eta}\left(1-\eta^{2}\right) \frac{\partial}{\partial \eta}+\beta^{2} \eta^{2}
$$

and $L_{\xi}$ the operator obtained from $L_{\eta}$ upon replacing $\eta$ by $i \xi$. Let $Y_{0 / 1}^{\text {even } / o d d}(\eta)$, with separation parameter $\mu_{e / o}(\beta)$, be one of the angular confluent Heun functions which satisfy the differential equation

$$
\left[L_{\eta}-\mu_{e / o}(\beta)\right] Y_{0 / 1}^{e v e n / o d d}(\eta)=0 .
$$

Now let $K(\xi, \eta)$ be a continuous function in $\eta$ and $\xi$, along with its first and second derivatives, and let $K(\xi, \eta)$ satisfy the following differential equation

$$
\left(L_{\xi}-L_{\eta}\right) K(\xi, \eta)=0
$$

in the range of $\eta$ and in the range of $\xi$, choosing the endpoints $-1 \leq \eta \leq 1$ such that

$$
\begin{aligned}
& \int_{-1}^{1}\left(Y_{0 / 1}^{\text {even } / \text { odd }}(\eta) L_{\eta} K(\xi, \eta)-K(\xi, \eta) L_{\eta} Y_{0 / 1}^{\text {even } / \text { odd }}(\eta)\right) d \eta \\
= & {\left[\left(1-\eta^{2}\right)\left(Y_{0 / 1}^{\text {even } / \text { odd }}(\eta) \frac{\partial K(\xi, \eta)}{\partial \eta}-K(\xi, \eta) \frac{\partial Y_{0 / 1}^{\text {even } / \text { odd }}(\eta)}{\partial \eta}\right)\right]_{\eta=-1}^{\eta=1} } \\
= & 0 .
\end{aligned}
$$


Then we have that

$$
X_{0}^{\text {even } / \text { odd }}(\xi)=\int_{-1}^{1} K(\xi, \eta) Y_{0 / 1}^{\text {even } / \text { odd }}(\eta) d \eta
$$

is a solution of the radial equation

$$
\left[L_{\xi}-\mu_{e / o}(\beta)\right] X_{0}^{e v e n / o d d}(\xi)=0,
$$

for all $\xi$ on the real line.

Proof: Applying the operator $L_{\xi}$ to Eqn. 3.56 we obtain

$$
\begin{aligned}
L_{\xi} X_{0}^{\text {even } / \text { odd }}(\xi) & =\int_{-1}^{1} L_{\xi} K(\xi, \eta) Y_{0 / 1}^{\text {even } / \text { odd }}(\eta) d \eta, \\
& =\int_{-1}^{1} Y_{0 / 1}^{\text {even } / \text { odd }}(\eta) L_{\eta} K(\xi, \eta) d \eta, \\
& =\int_{-1}^{1} K(\xi, \eta) L_{\eta} Y_{0 / 1}^{\text {even } / \text { odd }}(\eta) d \eta, \\
& =\mu_{e / o}(\beta) \int_{-1}^{1} K(\xi, \eta) Y_{0 / 1}^{\text {even } / \text { odd }}(\eta) d \eta, \\
& =\mu_{e / o}(\beta) X_{0}^{\text {even } / \text { odd }}(\xi) .
\end{aligned}
$$

The above proof can be found in [31] for the oblate (and prolate) spheroidal functions, but here we have shown that it is valid for the radial and angular confluent Heun functions. (This analysis actually shows a one-toone correspondence between the radial oblate spheroidal and radial confluent Heun functions that shall be explained in section 3.6.2.) If $\left(\nabla^{2}+k^{2}\right) \psi(\xi, \eta)$ $=0$ in oblate spheroidal coordinates and

$$
\psi(\xi, \eta)=K(\xi, \eta),
$$

then $K(\xi, \eta)$ satisfies Eqn. 3.54. If we express the Helmholtz equation in cylindrical coordinates, omitting azimuthal dependence again, then the equation satisfied by $K(\rho, z)$ is

$$
\left(\frac{\partial^{2}}{\partial \rho^{2}}+\frac{1}{\rho} \frac{\partial}{\partial \rho}+\frac{\partial^{2}}{\partial z^{2}}+k^{2}\right) K=0 .
$$

There are various solutions to Eqn. 3.59: one such solution is $\exp (i k z)$, which becomes $\exp (i \beta \xi \eta)$ in oblate spheroidal coordinates. 
There are other choices of Kernels involving Bessel, Hankel, or spherical Bessel functions, but the exponential is the simplest of them. We have now obtained the integral relations for the even and odd radial confluent Heun solutions. The integral relations for the radial solutions are given, in normalized form, by

$$
\begin{gathered}
X_{0}^{\text {even }}(\xi)=\frac{\int_{-1}^{1} \exp (i \beta \xi \eta) Y_{0 / 1}^{\text {even }}(\eta) d \eta}{\int_{-1}^{1} Y_{0 / 1}^{\text {even }}(\eta) d \eta}=\frac{\int_{0}^{1} \cos (\beta \xi \eta) Y_{0 / 1}^{\text {even }}(\eta) d \eta}{\int_{0}^{1} Y_{0 / 1}^{\text {even }}(\eta) d \eta} . \\
X_{0}^{\text {odd }}(\xi)=\frac{\int_{-1}^{1} \exp (i \beta \xi \eta) Y_{0 / 1}^{\text {odd }}(\eta) d \eta}{i \int_{-1}^{1} \beta \eta Y_{0 / 1}^{\text {odd }}(\eta) d \eta}=\frac{\int_{0}^{1} \sin (\beta \xi \eta) Y_{0 / 1}^{\text {odd }}(\eta) d \eta}{\int_{0}^{1} \beta \eta Y_{0 / 1}^{\text {odd }}(\eta) d \eta} .
\end{gathered}
$$

The normalization has been chosen so that $X_{0}^{\text {even }}(\xi)$ is unity at $\xi=0$, whereas the normalization of $X_{0}^{\text {odd }}(\xi)$ is determined by setting $d X_{0}^{\text {odd }}(\xi) /\left.d \xi\right|_{\xi=0}=1$. The asymptotic form of the even and odd radial solutions may now be derived by integration by parts of the expressions in Eqn. 3.60 and Eqn. 3.61. Thus we have that

$$
\begin{aligned}
X_{0}^{\text {even }}(\xi) & =\frac{\int_{0}^{1} \cos (\beta \xi \eta) Y_{0 / 1}^{\text {even }}(\eta) d \eta}{\int_{0}^{1} Y_{0 / 1}^{\text {even }}(\eta) d \eta}, \\
& =\frac{1}{\beta \xi} \frac{\int_{0}^{1} \frac{d}{d \eta}(\sin (\beta \xi \eta)) Y_{0 / 1}^{\text {even }}(\eta) d \eta}{\int_{0}^{1} Y_{0 / 1}^{\text {even }}(\eta) d \eta}, \\
\Rightarrow X_{0}^{\text {even }}(\xi) & \rightarrow \frac{Y_{0 / 1}^{\text {even }}(1)}{\int_{0}^{1} Y_{0 / 1}^{\text {even }}(\eta) d \eta} \frac{\sin (\beta \xi)}{\beta \xi}, \quad \beta \xi \rightarrow \infty .
\end{aligned}
$$

Comparison of the asymptotic expression in Eqn. 3.62 can be made with that found in Eqn. 3.47, from which we deduce that

$$
\begin{aligned}
U(\beta, 1) & =\sum_{n=0,2,4, \ldots}^{\infty}(-i)^{n} a_{n}(\beta) P_{n}(1), \\
& =\sum_{\substack{n=0,1,2, \ldots \\
\text { even }}}^{\infty}(-1)^{n} a_{2 n}(\beta) \\
& =\frac{Y_{0 / 1}^{\text {even }}(1)}{\int_{0}^{1} Y_{0 / 1}^{\text {even }}(\eta) d \eta}
\end{aligned}
$$


Performing the same analysis for the odd radial solution we find

$$
\begin{aligned}
X_{0}^{\text {odd }}(\xi) & =\frac{\int_{0}^{1} \sin (\beta \xi \eta) Y_{0 / 1}^{\text {odd }}(\eta) d \eta}{\int_{0}^{1} \beta \eta Y_{0 / 1}^{\text {odd }}(\eta) d \eta}, \\
& =\frac{-1}{\beta \xi} \frac{\int_{0}^{1} \frac{d}{d \eta}(\cos (\beta \xi \eta)) Y_{0 / 1}^{\text {odd }}(\eta) d \eta}{\int_{0}^{1} \beta \eta Y_{0 / 1}^{\text {odd }}(\eta) d \eta}, \\
\Rightarrow X_{0}^{\text {odd }}(\xi) & \rightarrow \frac{-Y_{0 / 1}^{\text {odd }}(1)}{\int_{0}^{1} \beta \eta Y_{0 / 1}^{\text {odd }}(\eta) d \eta} \frac{\cos (\beta \xi)}{\beta \xi}, \quad \beta \xi \rightarrow \infty .
\end{aligned}
$$

On comparing Eqn. 3.64 with Eqn. 3.49, we find that the coefficient in the asymptotic expression for the odd radial solution is

$$
\begin{aligned}
V(\beta, 1) & =-\sum_{n=1,3,5}^{\infty}(-i)^{n-1} b_{n}(\beta) P_{n}(1), \\
& =-\sum_{n=0,1,2}^{\infty}(-1)^{n} b_{2 n+1}(\beta), \\
& =\frac{-Y_{0 / 1}^{\text {odd }}(1)}{\int_{0}^{1} \beta \eta Y_{0 / 1}^{\text {odd }}(\eta) d \eta} .
\end{aligned}
$$

These relations hold for either of the even or odd angular confluent Heun functions. The coefficients $U(\beta, 1)$ and $V(\beta, 1)$ are simplified in the case where we use the angular confluent Heun function $Y_{1}^{\text {even/odd }}(\eta)$, since in this case their value at $\eta=1$ is exactly 1 . Thus the numerator in Eqn. 3.63 is 1 , while in Eqn. 3.65 it is -1 . For example, for $\beta=1, \mu_{e}(\beta)=\mu_{00}(\beta)$, and $\mu_{o}(\beta)=\mu_{01}(\beta), U(\beta, 1) \approx 1.1165$ and $V(\beta, 1) \approx-3.1202$. The even radial solution and its asymptotic form are shown in Fig. 3.2, whereas the odd radial solution and its asymptotic form are shown in Fig. 3.3.

In conclusion, we have shown that in the case of scalar beams, there are integral relations for the even and odd radial confluent Heun solutions in terms of the angular solutions. This has allowed the asymptotic nature of the radial solutions to be derived, which gives the result that the infinite alternating series of spherical Bessel coefficients reduces to the inverse of an integral of an angular confluent Heun function. This result can only be checked numerically using the correct $\mu_{e / o}(\beta)$ since an analytic expression for $\mu_{e / o}(\beta)$ is unavailable at this stage. 


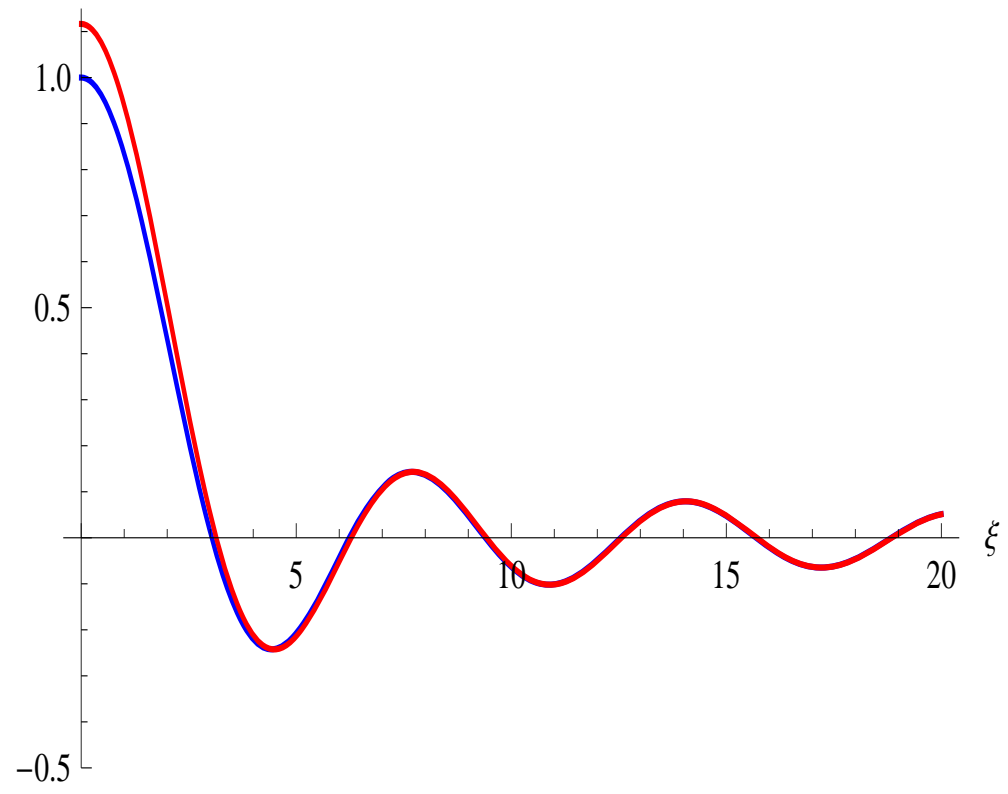

Figure 3.2: The even radial confluent Heun solution $X_{0}^{\text {even }}(\xi)$ (blue) and its asymptotic form (red), plotted for $\beta=1, \mu(\beta)=\mu_{00}(\beta)$.

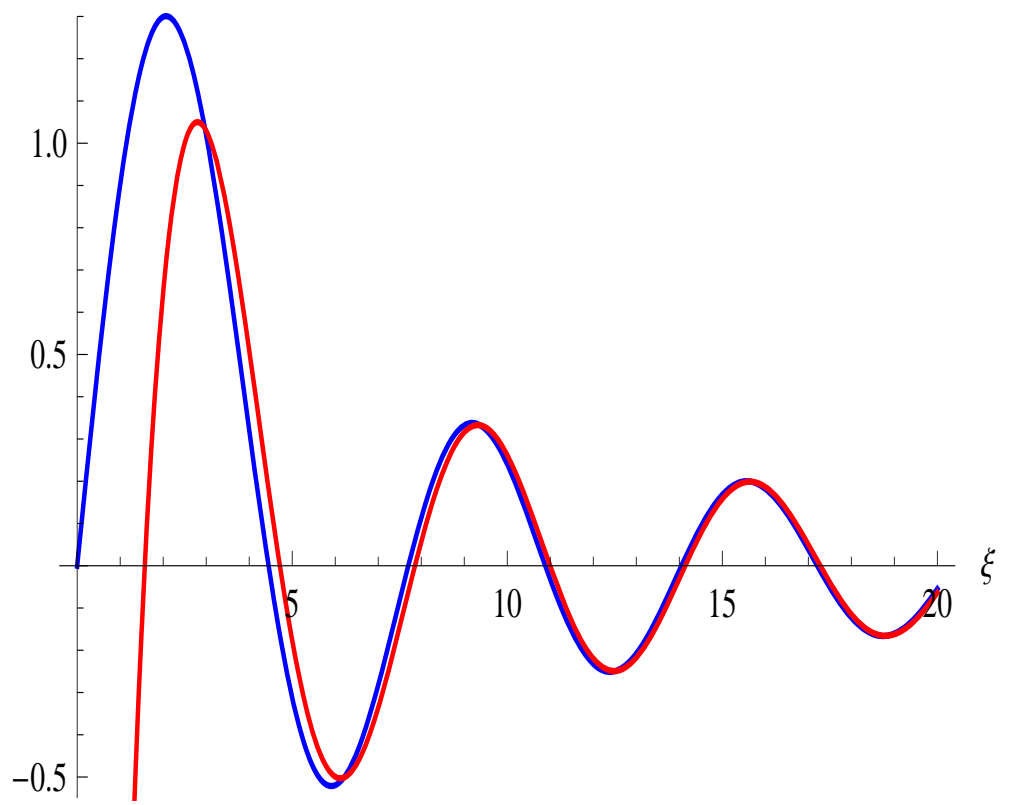

Figure 3.3: The odd radial confluent Heun solution $X_{0}^{\text {odd }}(\xi)$ (blue) and its asymptotic form (red), plotted for $\beta=1, \mu(\beta)=\mu_{01}(\beta)$. 


\subsection{Nonparaxial and paraxial scalar beams}

\subsubsection{Confluent Heun beams}

As a summary of what we have derived in the previous sections we have the following:

- Analytic solutions of the Helmholtz equation in terms of confluent Heun functions (sum of products over an odd radial times an odd angular plus an even radial times an even angular.)

- An analytic method of obtaining the even and odd separation parameters $\mu_{e / o}(\beta)$ by solving the Wronskian of two linearly dependent angular solutions equal to zero.

- Exact representation of the asymptotic form of the radial solutions. (We also have their expansions in terms of spherical Bessel functions which give the sums of the alternating series discussed previously.)

As noted earlier, the asymptotic forms of the two radial solutions requires that we use different separation parameters, $\mu_{e}(\beta)$ for $X_{0}^{\text {even }}(\xi)$ and $\mu_{o}(\beta)$ for $X_{0}^{\text {odd }}(\xi)$. Therefore we cannot construct a beam which is forward propagating in this manner. Nevertheless we shall investigate this type of solution since it remains an analytic solution of Helmholtz's equation.

Denote the scalar confluent Heun beam by

$$
\psi_{H}(\xi, \eta)=A(\beta) X_{0}^{\text {odd }}(\xi) Y_{1}^{\text {odd }}(\eta)+i B(\beta) X_{0}^{\text {even }}(\xi) Y_{1}^{\text {even }}(\eta),
$$

where $A(\beta)$ and $B(\beta)$ are normalization constants to be derived in order that the beam is forward propagating along the beam axis. They are not the $A(\beta)$ and $B(\beta)$ discussed in section 3.4. $A(\beta)$ and $B(\beta)$ are functions of $\beta$, since the asymptotic nature of the radial solutions is dependent on $\beta$ too. It is understood in this expression that all odd confluent Heun functions use $\mu_{01}(\beta)$, while all even confluent Heun functions use $\mu_{00}(\beta)$ as the separation parameter. 
Other confluent Heun beams can be constructed using different $\mu_{e}(\beta)$ and $\mu_{o}(\beta)$, but here we are interested in the lowest order confluent Heun beam, i.e. $\mu_{e}(\beta)=\mu_{00}(\beta)$ and $\mu_{o}(\beta)=\mu_{01}(\beta)$. The asymptotic nature of the scalar beam, as $\beta \xi \rightarrow \infty$, is

$$
\psi_{H}(\xi, \eta) \rightarrow A(\beta) V(\beta, 1) \frac{\cos (\beta \xi)}{\beta \xi} Y_{1}^{\text {odd }}(\eta)+i B(\beta) U(\beta, 1) \frac{\sin (\beta \xi)}{\beta \xi} Y_{1}^{\text {even }}(\eta)
$$

We require that the solutions are forward propagating along the beam axis, and so we set $\eta=1$. The asymptotic nature of the scalar beam solution can then be put in an explicit form as a superposition of positive and negative exponentials:

$$
\begin{aligned}
\psi_{H}(\xi, \eta=1) & \rightarrow A(\beta) V(\beta, 1) \frac{\cos (\beta \xi)}{\beta \xi}+i B(\beta) U(\beta, 1) \frac{\sin (\beta \xi)}{\beta \xi}, \quad \beta \xi \rightarrow \infty \\
& =\frac{1}{2 \beta \xi}(A V+B U) e^{i \beta \xi}+\frac{1}{2 \beta \xi}(A V-B U) e^{-i \beta \xi}
\end{aligned}
$$

To ensure forward propagation along the beam axis, the condition $A(\beta) V(\beta, 1)$ $=B(\beta) U(\beta, 1)$ must be satisfied. Therefore the scalar beam solution is

$$
\psi_{H}(\xi, \eta)=B(\beta)\left(\frac{U(\beta, 1)}{V(\beta, 1)} X_{0}^{\text {odd }}(\xi) Y_{1}^{\text {odd }}(\eta)+i X_{0}^{\text {even }}(\xi) Y_{1}^{\text {even }}(\eta)\right) .
$$

The parameter $B(\beta)$ determines the normalization of the beam and also the isophase surfaces. We shall normalize the beam so that at the origin, where the beam axis intersects the focal plane, $\left|\psi_{H}(\rho, z)\right|=\left|\psi_{H}(0,0)\right|=1$, which is satisfied by setting $B(\beta)=i$. The asymptotic nature of the scalar beam, on the $z$ axis, is then $i U(\beta, 1) e^{i \beta \xi} / \beta \xi=i U(\beta, 1) e^{i k z} / k z$.

The asymptotic form of the wave function, on the beam axis, is shown in Fig. 3.4 for a tightly focused $\beta=2$ scalar beam. The plot shows $\Re\left(\psi_{H}\right)$ compared to $-U(2,1) \sin (k z) / k z$ and $\Im\left(\psi_{H}\right)$ compared to $U(2,1) \cos (k z) / k z$. In both cases the agreement is very good, and so the scalar beam constructed here is forward propagating along the beam axis, for large $k z$. 


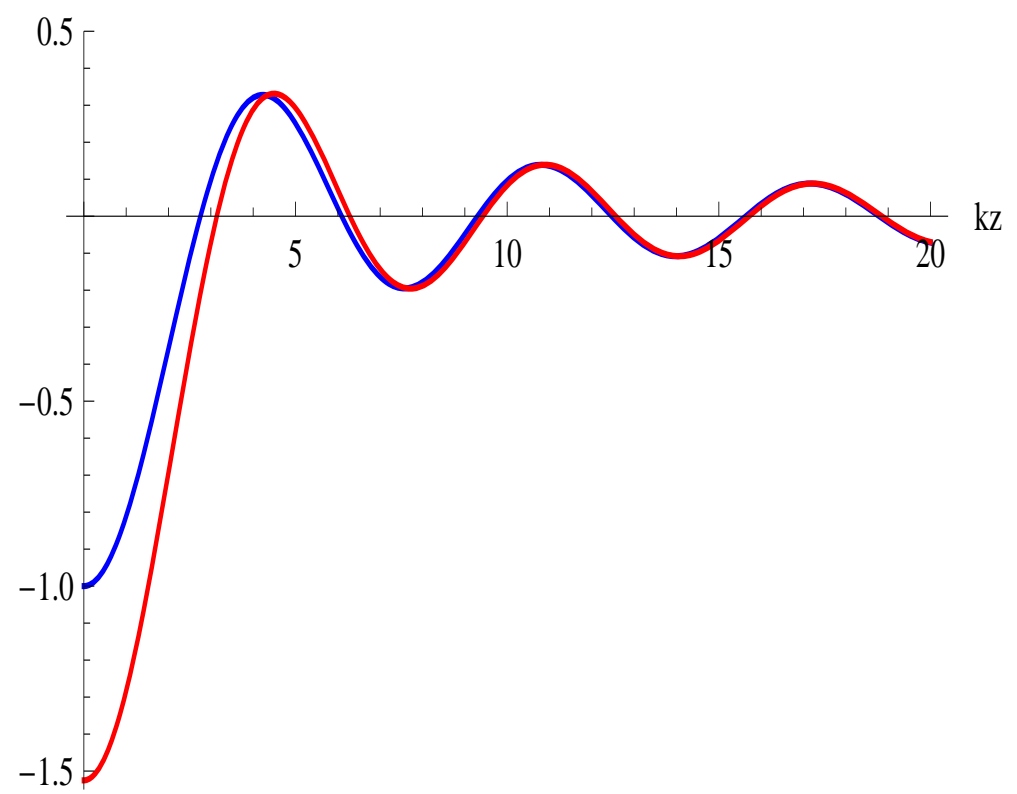

(a) Real part of $\psi_{H}$.

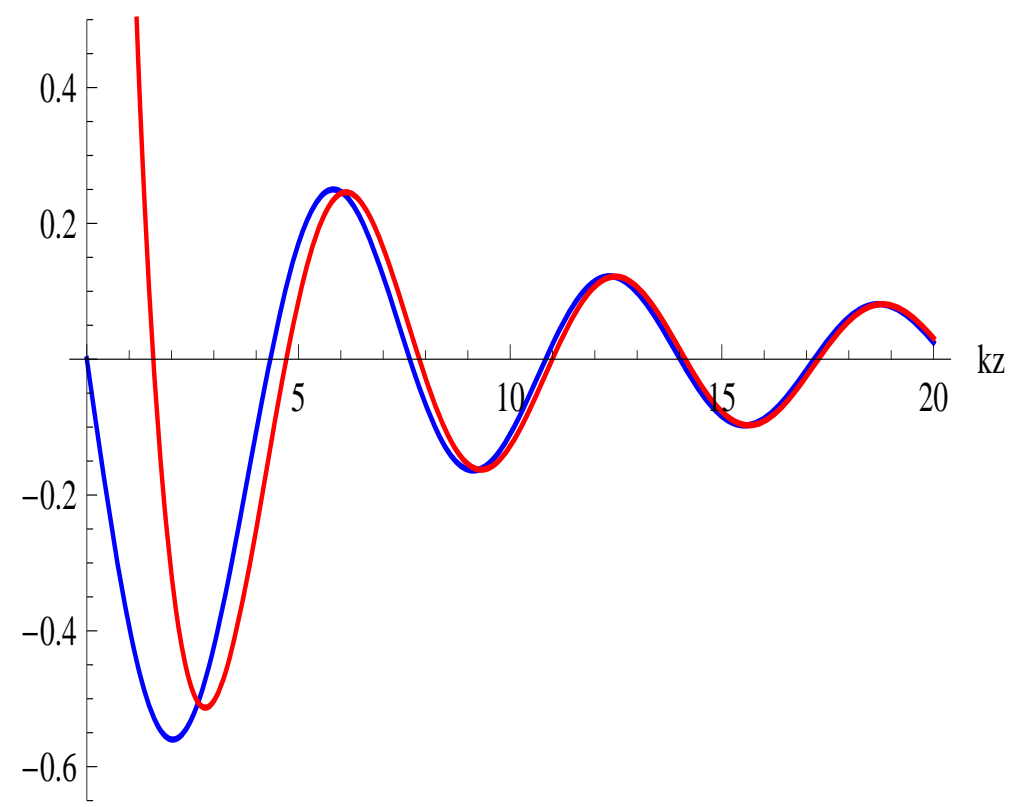

(b) Imaginary part of $\psi_{H}$.

Figure 3.4: The real and imaginary parts of the scalar beam $\psi_{H}$ (blue), along the beam axis, plotted for $\beta=2$. The asymptotic forms $-U(2,1) \sin (k z) / k z$ and $U(2,1) \cos (k z) / k z$ (red) are also shown. As the beam has its real and imaginary components asymptotic to these expressions, it is forward propagating along the beam axis, for large $k z$. 
The scalar beams constructed here are exact solutions of the Helmholtz equation. There has been another set of exact beam wave functions proposed by Ulanowski and Ludlow [15], which take the form of spherical Bessel beams. The properties of these beams have been extensively investigated by Lekner $[16,47,50]$. The wave function of such beams is given by

$$
\psi_{m n}(\rho, z)=j_{n}(k R) P_{n}^{m}\left(\frac{z-i b}{R}\right) e^{ \pm i m \phi}, \quad R^{2}=\rho^{2}+(z-i b)^{2} .
$$

These beams represent the separable solutions of the Helmholtz equation in spherical coordinates, however, they have been shifted along the $z$ axis. The lowest of these set of beams is $\psi_{00}$ and is given by $\sin (k R) / k R$. This solution is the (non-singular) generalization of the exact solution $\exp (i k R) / R$, which was initially proposed by Deschamps [11], as mentioned in the introduction. (The problem with the complex source point solution of Deschamps is that it has a singularity in the focal plane $z=0$ on the circle $\rho=b$. The spherical Bessel beam $\psi_{00}$ does not have this singularity, since it is constructed through a superposition of incoming and outgoing waves.)

In Fig. 3.5 the probability densities for both the confluent Heun beam wave function $\psi_{H}$, given in Eqn. 3.69, and the $\psi_{00}$ beam wave function of Eqn. 3.70 are shown for a tightly focused $\beta=2$ scalar beam. Note the spherical Bessel beam has been multiplied by $\beta / \sinh (\beta)$ to normalize it to unity at the origin $(\rho=z=0)$. In this figure the probability current density $\mathbf{J}$, given by the field vectors, has also been shown. 


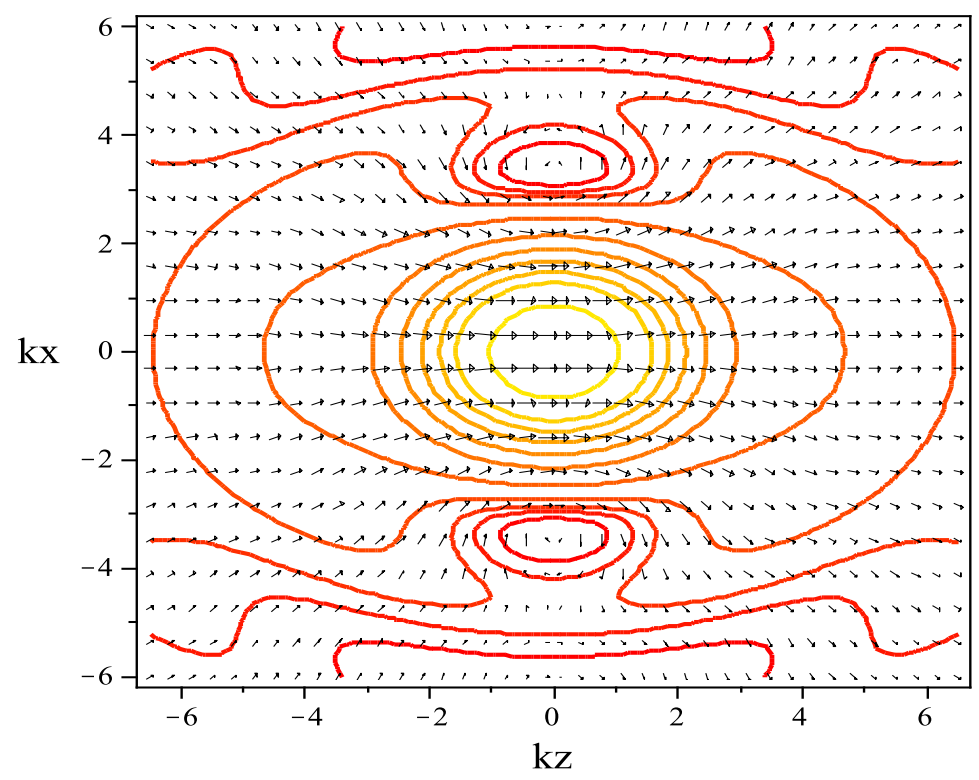

(a) confluent Heun beam $\psi_{H}$.

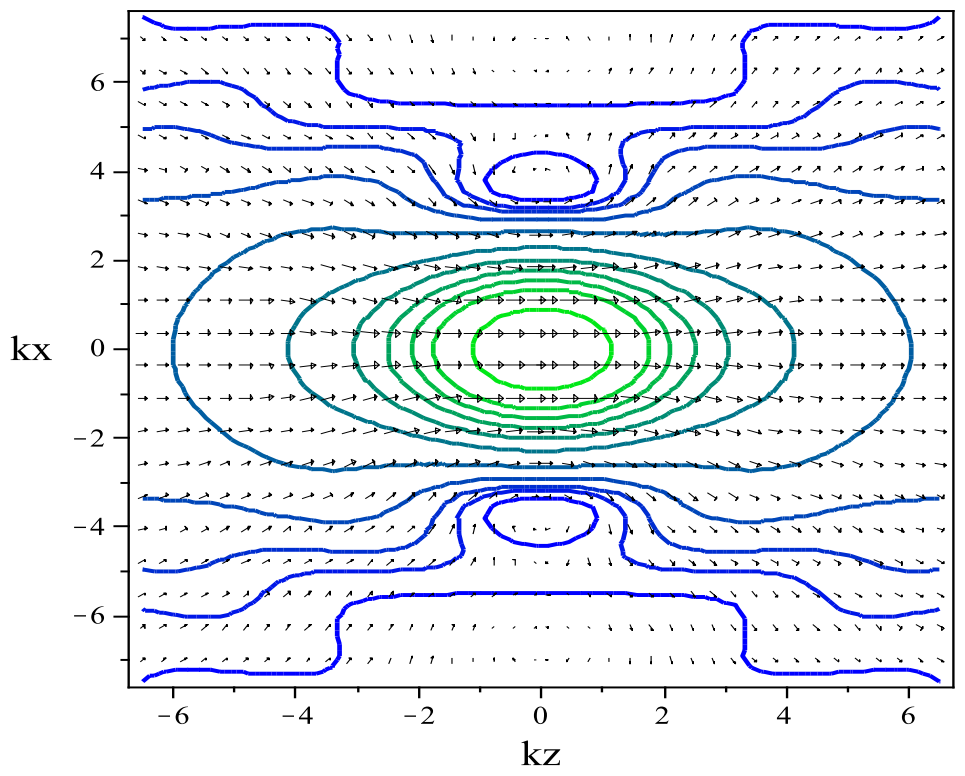

(b) spherical Bessel beam $\psi_{00}$.

Figure 3.5: The probability densities $|\psi|^{2}$ for the confluent Heun beam $\psi_{H}$, and for the spherical Bessel beam $\psi_{00}$, plotted for $\beta=2$. The contours are at $[0.01$, $0.02,0.03,0.05, .1, .2, .3, .4, .5, .6, .8]$, with the maximum occurring at the origin. The arrows show $\left[J_{z}, J_{x}\right]$, which indicates the probability current density. The three dimensional picture is obtained by rotating the figure about the $z$ axis. 
The plots of these two beam wave functions show remarkable similarity. In the focal plane $(z=0)$ the zeros of the two wave functions almost coincide and their peaks along this axis also nearly coincide. We can understand this similarity by analysis of the terms in each beam. In the focal plane we have $\eta=0$ for $\rho \geq b$, while for $\rho \leq b, \xi=0$. Examining the odd product in the confluent Heun beam we have $Y_{1}^{\text {odd }}(0)=0$ for $\rho \geq b$, while for $\rho \leq b$, $X_{0}^{\text {even }}(0)=0$. Thus, the product $X_{0}^{\text {odd }}(\xi) Y_{1}^{\text {odd }}(\eta)$ is always identically zero in the focal plane. It is only the even terms in the confluent Heun beam which are present in the focal plane.

Hence when $\rho \geq b(z=0)$ the confluent Heun beam probability density is asymptotic to $Y_{1}^{\text {even }}(0)^{2} U(\beta, 1)^{2} \sin ^{2}(\beta \xi) / \beta^{2} \xi^{2}$, whereas the (normalized) spherical Bessel beam probability density has the form $\sin ^{2}(\beta \xi) / \sinh ^{2}(\beta) \xi^{2}$. Therefore, in the focal plane the two expressions have the same $\xi$ dependence (asymptotically), but with different amplitudes dependent on $\beta$. This result explains why the zeros of both beams almost coincide, with the zeros of the spherical Bessel beam occurring at $k \rho=\sqrt{\beta^{2}+n^{2} \pi^{2}}$. In the region $\rho \leq b$, with $z=0$, the (normalized) spherical Bessel beam probability density is given by $\sinh ^{2}(\beta \eta) / \eta^{2} \sinh ^{2}(\beta)$, and if we use a spherical Bessel expansion for $Y_{0}^{\text {even }}(\eta)$, we find $\left|\psi_{H}\right|^{2}$ has leading term equal to $Y_{1}^{\text {even }}(0)^{2} \sinh ^{2}(\beta \eta) / \beta^{2} \eta^{2}$. In the focal plane the even terms of the confluent Heun beam give it similar features to the spherical Bessel beam $\psi_{00}$.

There is, however, a difference in the beam solutions along the beam axis. Whereas the (normalized) spherical Bessel beam probability density is $\left[\sin ^{2}(\beta \xi)+\sinh ^{2}(\beta)\right] /\left[\left(\xi^{2}+1\right) \sinh ^{2}(\beta)\right]$ along the $\eta=1$ axis, the confluent Heun beam probability density is asymptotic to $U(\beta, 1)^{2} / \beta^{2} \xi^{2}$. This corresponds to the difference in the wavefronts at points far along the beam axis. One possible reason for this difference is due to the fact that the spherical Bessel beams are not forward propagating, whereas along the beam axis the confluent Heun beam is.

Lekner [16] has shown that the spherical Bessel beam $\psi_{00}$ is one which is not physically realizable. A physical particle beam must have a finite probability content in a transverse slice of the beam. However, Lekner showed that the normalization integral of the $\psi_{00}$ beam is logarithmically divergent. 
Therefore, this indicates that the confluent Heun beam constructed here is not realizable either. For instance, the normalization integral in a crosssection of the beam is

$$
N=\int_{0}^{\infty} \rho|\psi|^{2} d \rho=\int_{0}^{b} \rho|\psi|^{2} d \rho+\int_{b}^{\infty} \rho|\psi|^{2} d \rho .
$$

On converting to oblate spheroidal coordinates, where $\rho d \rho=b^{2} \xi d \xi$, the second integral in Eqn. 3.71 (in the focal plane $z=0$ ) is

$$
\int_{b}^{\infty} \rho|\psi|^{2} d \rho=b^{2} \int_{0}^{\infty} \xi|\psi|^{2} d \xi .
$$

In the focal plane $(z=0)$, for $\rho \geq b$, only the even confluent Heun product is non-zero for the confluent Heun beam. The asymptotic behaviour of the probability density of this beam is $Y_{1}^{\text {even }}(0)^{2} U(\beta, 1)^{2} \sin ^{2}(\beta \xi) / \beta^{2} \xi^{2}$, thus the contribution to the normalization integral from Eqn. 3.72 is

$$
b^{2} \int_{0}^{\infty} \xi|\psi|^{2} d \xi \rightarrow b^{2} Y_{1}^{e v e n}(0)^{2} U(\beta, 1)^{2} \int_{0}^{\infty} \xi \frac{\sin ^{2}(\beta \xi)}{\beta^{2} \xi^{2}} d \xi,
$$

which is logarithmically divergent, for any $\beta$ (paraxial or not).

The confluent Heun beam has similar nonphysical properties, such as a divergent normalization integral and backward propagating elements, as the spherical Bessel beam $\psi_{00}$, but with an extra amount of complexity by way of introduction of confluent Heun functions. This result is due to the fact that we have used two separation parameters, and consequently although this allows correct asymptotic behaviour along the beam axis, it does not allow physical behaviour in the focal plane.

Lekner [51] has commented that the wave function $\psi_{00}$ may be useful in high aperture situations for small $\beta$ where it is an improvement on the Gaussian approximation. This would most likely be applicable to the confluent Heun beam solution too.

It is well-known that under certain conditions the Gaussian beam gives an approximate representation of a scalar beam. However, for nonparaxial (small $\beta$ ) scalar beams the Gaussian approximation breaks down. Therefore, we give a comparison of the confluent Heun beam with the Gaussian beam in both the nonparaxial ( mall $\beta$ ) case, and also the paraxial (large $\beta$ ) case. 
The Gaussian beam wave function is given by

$$
\psi_{G}=\frac{b}{b+i z} \exp \left[i k z-\frac{k \rho^{2}}{2(b+i z)}\right] .
$$

By considering $\psi_{G}^{-1} \nabla^{2} \psi_{G}$ the errors in the Gaussian approximation can be found. Instead of $-k^{2}$ we obtain $-k^{2}$ times

$$
1+\frac{2}{k^{2}(b+i z)^{2}}-\frac{2 \rho^{2}}{k(b+i z)^{3}}+\frac{\rho^{4}}{4(b+i z)^{4}} .
$$

The errors introduced in the Gaussian approximation are thus negligible in the regions where both $k^{2}\left(b^{2}+z^{2}\right)>>1$ and $b^{2}+z^{2}>>\rho^{2}$. In the focal plane $(z=0) \beta^{2}>>1$ and $\beta^{2}>>(k \rho)^{2}$ for the Gaussian approximation to hold [16]. The Gaussian beam is an exact solution of the paraxial Helmholtz equation, but not the full Helmholtz equation, and its validity relies on assuming large $\beta$ and small $k \rho$ compared to $\beta$. Therefore when $\beta$ is equal to unity or smaller the paraxial approximation will fail in the beam waist region $|z| \leq b$.

In Fig. 3.6 the probability densities for the confluent Heun beam $\psi_{H}$, the spherical Bessel beam $\psi_{00}$, and the Gaussian beam $\psi_{G}$ have been plotted along the beam axis. In one case for nonparaxial beams with $\beta=1$, and in the other case paraxial beams with $\beta=1 / 0.14$. The nonparaxial case shows a markedly different probability density for the confluent Heun beam in comparison with the Gaussian beam, particularly when $k|z| \approx \beta$. The confluent Heun beam is an exact solution in the nonparaxial regime, and here we observe the disagreement with the non-exact Gaussian beam.

On the beam axis $(\rho=0)$ the probability density of the Gaussian beam is $\beta^{2} /\left[\beta^{2}+(k z)^{2}\right]$, whereas the confluent Heun beam is asymptotic to $U(\beta, 1)^{2} /(k z)^{2}$. Along the beam axis, for $k z>>\beta$, the confluent Heun beam has the same $z$ dependence as the Gaussian beam, but with a different amplitude dependent on $\beta$. In section 3.6 .2 we shall show that $U(\beta, 1) \rightarrow \beta$, for large $\beta$, so that the confluent Heun beam is asymptotic to the Gaussian beam along the beam axis. When $\rho=0$, the spherical Bessel beam probability density is $\beta^{2}\left[\sin ^{2}(k z)+\sinh ^{2}(\beta)\right] /\left[\sinh ^{2}(\beta)\left((k z)^{2}+\beta^{2}\right)\right]$, which is also asymptotic to the Gaussian beam, for $k z>>\beta$, in the large $\beta$ limit. 


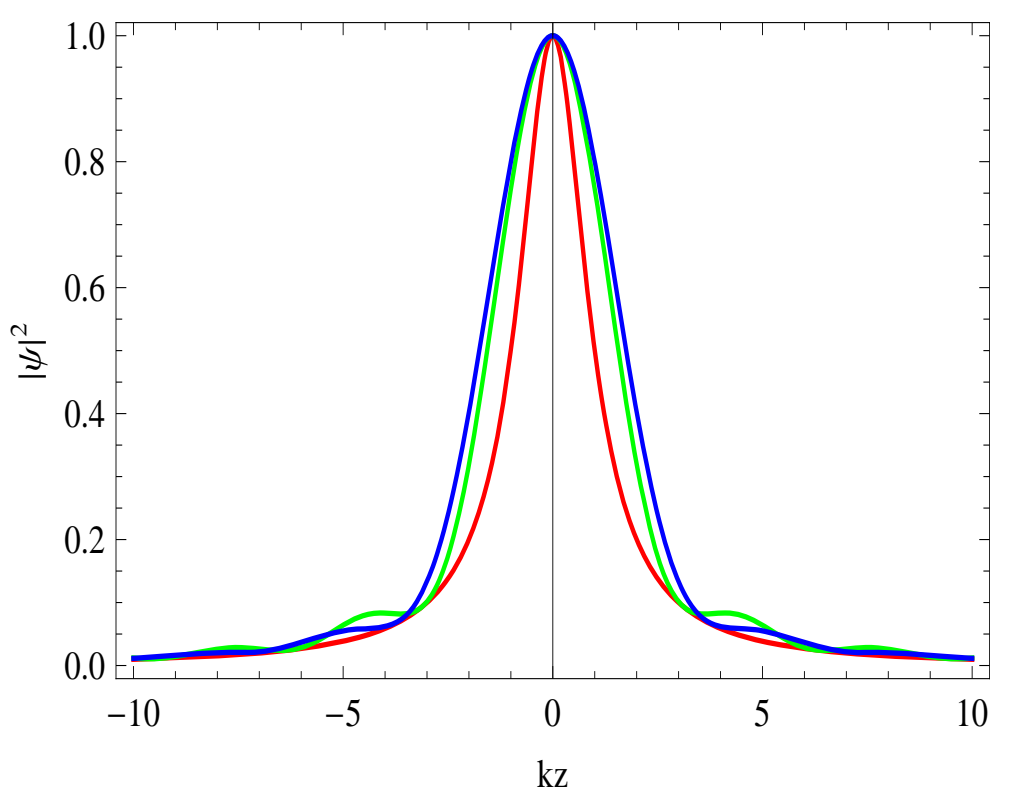

(a) Nonparaxial case, $\beta=1$.

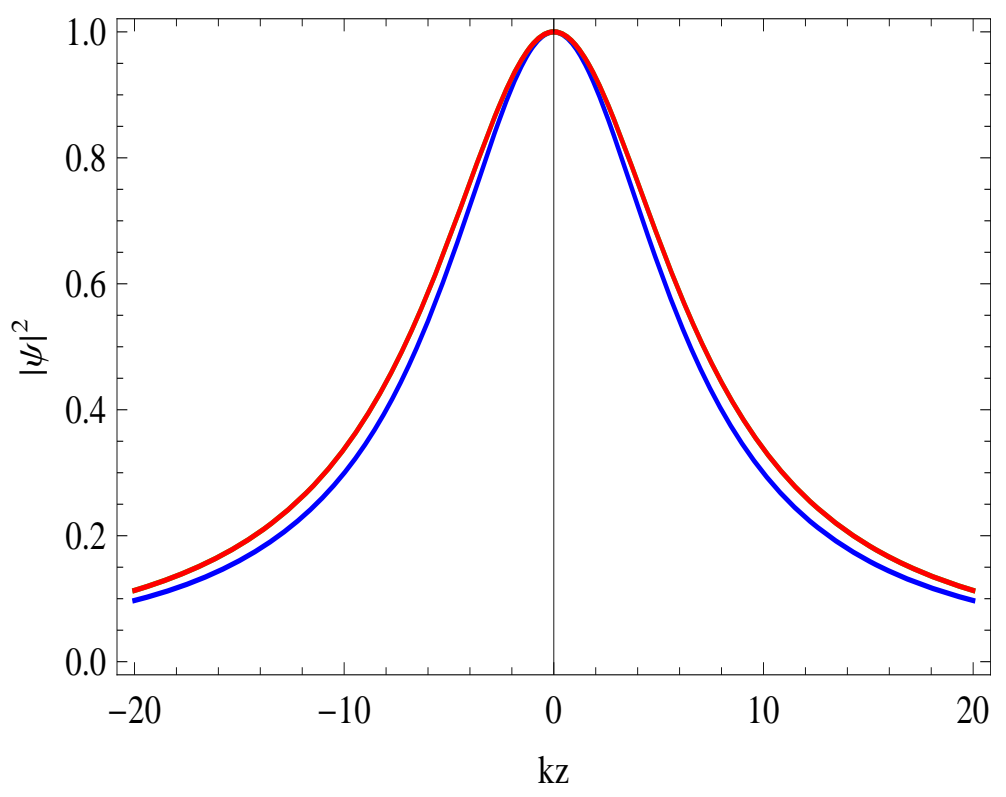

(b) Paraxial case, $\beta=1 / 0.14$.

Figure 3.6: The probability densities $|\psi|^{2}$ for the exact confluent Heun beam $\psi_{H}$ (blue), the exact spherical Bessel beam $\psi_{00}$ (green), and the approximate Gaussian beam $\psi_{G}$ (red), in the cases where $\beta=1$ and $\beta=1 / 0.14$. In both cases the plot is along the beam axis $\eta=1$. The upper plot shows the nonparaxial case of a small $\beta$, tightly focused beam, while the lower plot shows a large $\beta$, weakly focused beam. 
Fig. 3.7 shows the probability densities, in the focal plane $(z=0)$, for the confluent Heun beam $\psi_{H}$, the spherical Bessel beam $\psi_{00}$, and the Gaussian beam $\psi_{G}$. In the nonparaxial case, where $\beta=1$, the figure shows that the confluent Heun beam has almost coincident zeros and maxima with the spherical Bessel beam. This result has been explained earlier in relation to the contour plots of these probability densities in Fig. 3.5.

The paraxial case, where $\beta=1 / 0.14$, again shows that the confluent Heun beam has almost coincident zeros and maxima as the spherical Bessel beam. This being due to both beams having the same $\xi$ dependence (asymptotically). In this large $\beta$ case, Fig. 3.7 shows that, near the origin, i.e. $k \rho<<\beta$, the Gaussian beam and the confluent Heun beam have similar characteristics. However, for the Gaussian beam, the probability density is $\exp \left(-(k \rho)^{2} / \beta\right)$, which is normalizable, whereas the confluent Heun beam probability density is asymptotic to $Y_{1}^{\text {even }}(0)^{2} U(\beta, 1)^{2} \sin ^{2}(\beta \xi) / \beta^{2} \xi^{2}$, which is not normalizable.

The confluent Heun beam has a divergent normalization integral (in the focal plane), and off the beam axis, backward propagating elements. The Gaussian beam has a convergent normalization integral and is forward propagating. Thus, the confluent Heun beam is not the nonparaxial generalization of the Gaussian beam.

April [52] has shown that the spherical Bessel beam $\psi_{00}$ is not the nonparaxial generalization of $\psi_{G}$. Rather, certain linear combinations of the spherical Bessel beams are required to produce the Gaussian beam, in the large $\beta$ limit. (The linear combination of the spherical Bessel beams, however, still has the problem of backward propagation.)

This section has derived exact nonparaxial beam solutions in terms of confluent Heun functions. However, the beams have the problem of a divergent normalization integral and backward propagating elements, which a physical beam would not have. In the next section we shall construct scalar beams without these problems. 


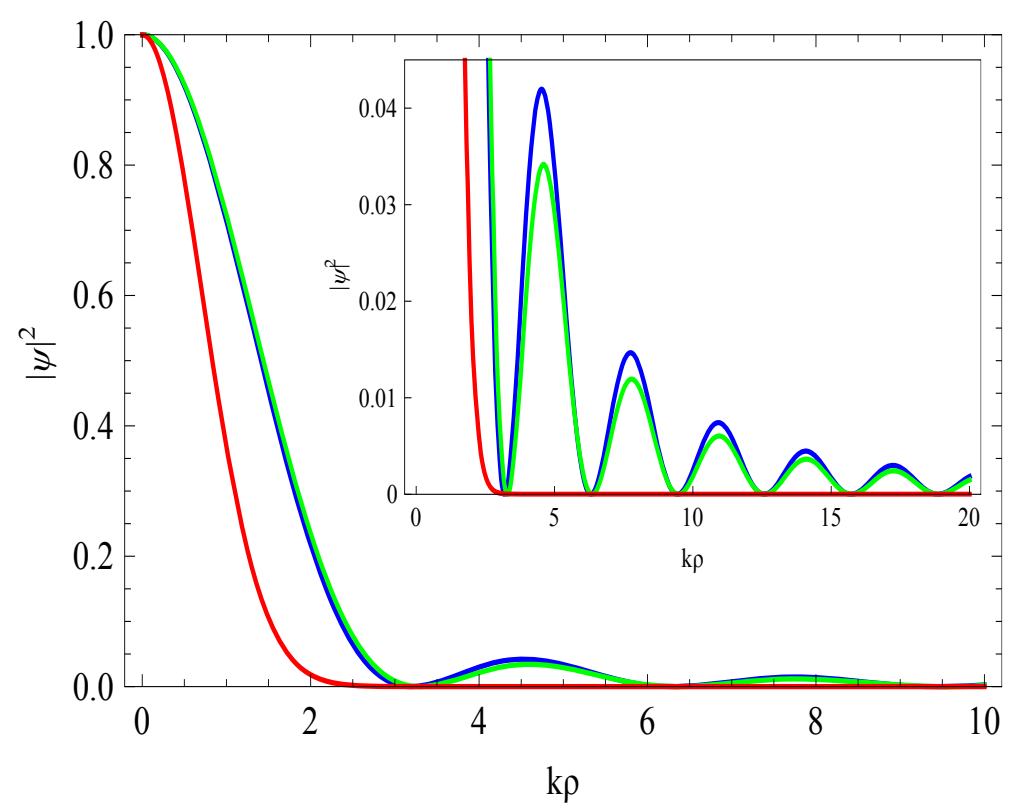

(a) Nonparaxial case, $\beta=1$.

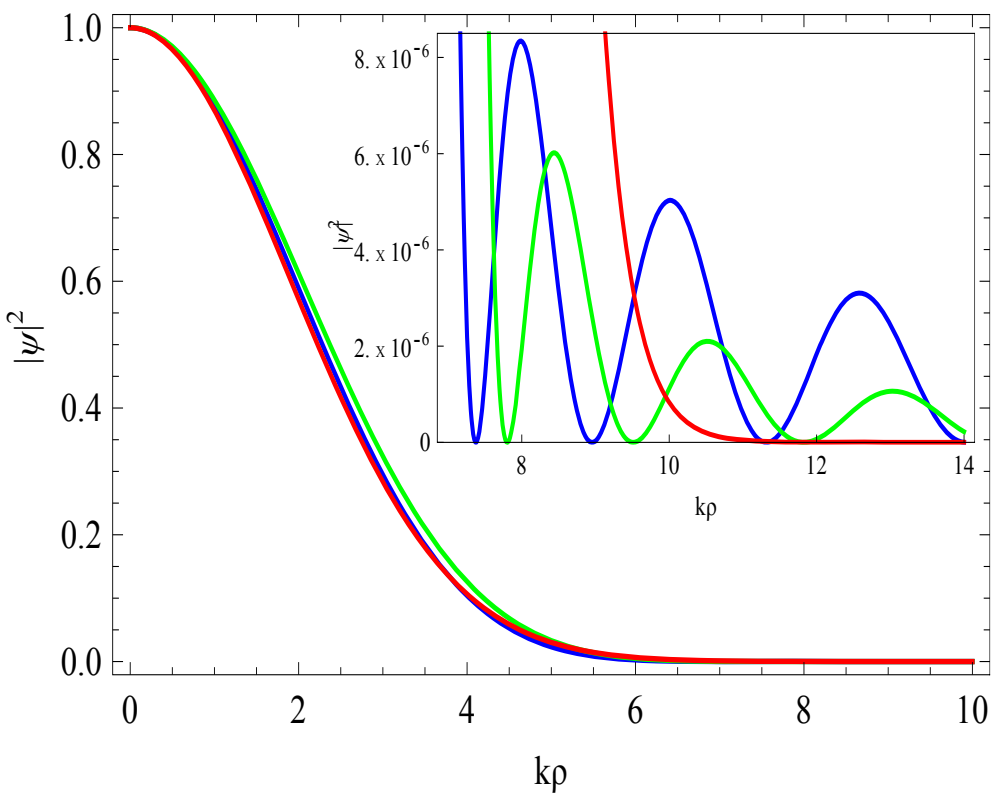

(b) Paraxial case, $\beta=1 / 0.14$.

Figure 3.7: The probability densities $|\psi|^{2}$ for the exact confluent Heun beam $\psi_{H}$ (blue), the spherical Bessel beam $\psi_{00}$ (green), and the approximate Gaussian beam $\psi_{G}$ (red), in the cases where $\beta=1$ and $\beta=1 / 0.14$. In both cases the plot is in the focal plane $z=0$. The upper plot shows the nonparaxial case of a small $\beta$, tightly focused beam, while the lower plot shows a large $\beta$, weakly focused beam. 


\subsubsection{Oblate spheroidal beams}

The scalar beam solutions proposed in Eqn. 3.69 do not represent physical free space scalar beams for two reasons. They are not forward propagating off the beam axis and they also have a logarithmically divergent normalization integral in the focal plane. Therefore, physical beams cannot be constructed from such solutions, since infinite energy would be required. The aim of this section is to investigate an analytic solution of Helmholtz's equation which satisfies the necessary physical requirements of forward propagating wavefronts, and a convergent normalization integral.

The problem with the confluent Heun beam is that it contains two separation of variable parameters, $\mu_{e}(\beta)$ and $\mu_{o}(\beta)$. In order for the beam to be forward propagating we require even and odd radial solutions whose asymptotic expansions are known when the same $\mu(\beta)$ is used for both. In this way a single angular solution may be used. Fortunately such functions are known. They are known as radial spheroidal functions. In the beam case we require the radial oblate, and angular oblate spheroidal functions. The properties of these functions are given in great detail in [31], but here we give their most important properties.

The radial equation is given by

$$
\left(\xi^{2}+1\right) \frac{d^{2} R}{d \xi^{2}}+2 \xi \frac{d R}{d \xi}+\left(\beta^{2} \xi^{2}+\frac{m^{2}}{\xi^{2}+1}+\mu\right) R=0
$$

where we have used the variable $R(\beta, \xi)$ which is standard for the radial oblate spheroidal functions. Two linearly independent solutions to Eqn. 3.76 are given by

$$
\begin{aligned}
& R(\beta, \xi)=R_{m n}^{(1)}(\beta, \xi) \\
& R(\beta, \xi)=R_{m n}^{(2)}(\beta, \xi)
\end{aligned}
$$

$R_{m n}^{(1)}(\beta, \xi)$ is known as the radial oblate spheroidal function of the first kind, while $R_{m n}^{(2)}(\beta, \xi)$ is known as the radial oblate spheroidal function of the second kind. $R_{m n}^{(1)}(\beta, \xi)$ is even if $n-m$ is even, whereas $R_{m n}^{(2)}(\beta, \xi)$ is even when $n-m$ is odd. We shall use the notation $R_{m n}^{(3)}(\beta, \xi)$ to represent $R_{m n}^{(1)}(\beta, \xi)+i R_{m n}^{(2)}(\beta, \xi)$. 
The asymptotics of these functions are well-known, and as $\beta \xi \rightarrow \infty$, are

$$
\begin{aligned}
& R_{m n}^{(1)}(\beta, \xi) \rightarrow \frac{1}{\beta \xi} \cos \left[\beta \xi-\frac{\pi}{2}(n+1)\right], \\
& R_{m n}^{(2)}(\beta, \xi) \rightarrow \frac{1}{\beta \xi} \sin \left[\beta \xi-\frac{\pi}{2}(n+1)\right] .
\end{aligned}
$$

The radial oblate spheroidal functions are defined similarly to the radial confluent Heun functions, i.e. through an expansion of even or odd spherical Bessel functions, and later a one-to-one relation between the two shall be given. The importance of the radial spheroidal functions is that in the case $\mu(\beta)=\mu_{e}(\beta)$, or $\mu(\beta)=\mu_{o}(\beta)$, the asymptotics of both are known.

The angular equation is given by

$$
\left(1-\eta^{2}\right) \frac{d^{2} S}{d \eta^{2}}-2 \eta \frac{d S}{d \eta}+\left(\beta^{2} \eta^{2}-\frac{m^{2}}{1-\eta^{2}}-\mu\right) S=0,
$$

where we have used the variable $S(\beta, \eta)$ which is standard for the angular oblate spheroidal functions. For a given $\beta$ only a certain set $\mu_{m n}(\beta)(m, n=$ $0,1,2, \ldots)$ give physical solutions $S(\eta)$ which are finite at $\eta^{2}=1$.

The scalar beam solutions, with a single separation parameter, are

$$
\psi_{m n}(\xi, \eta)=\left[R_{m n}^{(1)}(\beta, \xi)+i R_{m n}^{(2)}(\beta, \xi)\right] S_{m n}(\beta, \eta) e^{i m \phi} .
$$

The wave function in Eqn. 3.82 was initially proposed by Rodríguez-Morales and Chávez-Cerda [17]. However, Lekner and Boyack [18] have demonstrated that only a subset of those solutions may represent physical scalar beams. Only those solutions with $n-m$ odd can be physical, but once this condition is imposed, it leads to a wave function with a discontinuous $z$ derivative, at $z=0$, both inside and outside the circle $\rho=b$. (We shall not discuss the spherical Bessel beam solutions, other than the simplest solution $j_{0}(k R)$, and so there should not arise any confusion with Eqn. 3.70.) Instead of using the solution given in Eqn. 3.82, initially proposed in [17], we shall use the modified solution

$$
\psi_{m n}(\xi, \eta)=i\left[R_{m n}^{(1)}(\beta, \xi)+i R_{m n}^{(2)}(\beta, \xi)\right] S_{m n}(\beta, \eta) e^{i m \phi} .
$$

Eqn. 3.83 is still a solution of the Helmholtz equation. 
The introduction of the imaginary number $i$ is necessary for the isophase surfaces to have the correct symmetry about the focal plane, as will be shown. In this section we shall now give the first thorough investigation of the beam solutions in Eqn. 3.83 and show that, if $n-m$ is odd, they meet three necessary physical requirements of free space scalar beams. Later we shall point out a flaw in this beam wave function which makes it nonphysical. The next section shall prove a more general result about whether the proposed separable spheroidal beams are physical.

\section{Forward propagation}

Far from the origin, when $r=\left(\rho^{2}+z^{2}\right)^{1 / 2}=b\left(\xi^{2}-\eta^{2}+1\right)^{1 / 2}$ is large compared to both the scale length $b$ and to $k^{-1}$, i.e. at large $\xi$, the wave function $\psi_{m n}(\xi, \eta)$ has the correct forward propagating form:

$$
\psi_{m n}(\xi, \eta) \rightarrow \frac{i}{\beta \xi} \exp \left\{i\left[\beta \xi-\frac{\pi}{2}(n+1)\right]\right\} S_{m n}(\beta, \eta) e^{i m \phi} .
$$

Thus, the spheroidal wave functions are manifestly forward propagating, for any value of the separation parameter.

\section{Isophase surfaces}

Consider secondly the isophase surfaces associated with the wave function in Eqn. 3.83. $R_{m n}^{(1,2)}$ and $S_{m n}$ are real functions. Thus the phase of the wave function in Eqn. 3.83 is $m \phi$ plus a function of $\xi$ :

$$
P_{m n}(\beta, \xi, \phi)=m \phi+\arctan \left(-\frac{R_{m n}^{(1)}(\beta, \xi)}{R_{m n}^{(2)}(\beta, \xi)}\right) .
$$

At fixed $\phi=\phi_{0}$ (or when $m=0$ at any $\phi$ ) the phase is constant on the ellipses $\xi=\xi_{0}, \phi=\phi_{0}$ defined in Eqn. 3.15. On the ellipse $\xi=\xi_{0}$, $\phi=\phi_{0}$ the phase is $P_{m n}\left(\beta, \xi_{0}, \phi_{0}\right)$, and on the left of the focal plane $(z=0)$ the phase on the ellipse $\xi=-\xi_{0}, \phi=\phi_{0}$ is $P_{m n}\left(\beta,-\xi_{0}, \phi_{0}\right)$. The ratio $R_{m n}^{(1)}(\beta, \xi) / R_{m n}^{(2)}(\beta, \xi)$ is odd in $\xi$ for any $m, n$, and so the phase has a discontinuity of $2 \arctan \left(-R_{m n}^{(1)}(\beta, \xi) / R_{m n}^{(2)}(\beta, \xi)\right)$ in the plane $z=0$. 
The phase of any complex function $\psi$ can only be discontinuous where $\psi$ $=0$. All of the ellipsoids intersect the $z=0$ plane at $\rho \geq b$. Thus we need wave functions $\psi$ that are zero for all $\rho \geq b$ on $z=0$; that is, $\psi$ must be zero when $\eta=0$ (see Fig. 3.1). The angular functions $S_{m n}(\beta, \eta)$ are zero when $\eta=0$ if $n-m$ is odd, and non-zero if $n-m$ is even, see [30] or [31]. Therefore, $n-m$ odd is a necessary condition for physical behaviour of the phase of $\psi_{m n}(\xi, \eta)$.

The isophase surfaces also illustrate the need for the factor $i$ in Eqn. 3.83: $z=0$ is a plane of symmetry and therefore in this plane the $m=0$ beam wavefront is planar. The associated phase of the beam must therefore be zero when $\xi=0$. The radial oblate function which is zero when $\xi=0$ is the radial function of the first kind, thus the factor $i$ is needed in order for the phase to have the correct form in the focal plane.

For odd $n-m$ the wave functions have isophase surfaces that, at fixed $\phi$, intersect ellipses. For $m=0$ (and odd $n$ ) the isophase surfaces are halfellipsoids, joining at $z=0$ to a half-ellipsoid of the opposite phase. It is interesting to compare this behaviour with the $m=0$ Gaussian beam, given in Eqn. 3.74, which is an approximate solution of the Helmholtz equation valid in the paraxial regime, and with the spherical Bessel beam $j_{0}(k R)$, defined in Eqn. 3.70.

In Fig. 3.8 the isophase surfaces of the Gaussian beam $\psi_{G}$, the spherical Bessel beam $j_{0}(k R)$, and the oblate spheroidal beam $\psi_{01}(\xi, \eta)$ are shown. In the Gaussian case the isophase surfaces are all asymptotic to the $z=0$ focal plane as $\rho \rightarrow \infty$, i.e. as the wave function $\psi_{G} \rightarrow 0$. The $j_{0}(k R)$ beam isophase surfaces, in contrast, converge on to the zeros of $\sin \beta(\xi-i \eta)$, which on the $z=0, \rho \geq b$ part of the focal plane lie on the circles $\rho_{\nu}=$ $b\left[(\nu \pi / \beta)^{2}+1\right]^{1 / 2}, \nu=1,2, \ldots$ (except when the phase is an integer multiple of $\pi$, see [47].) 

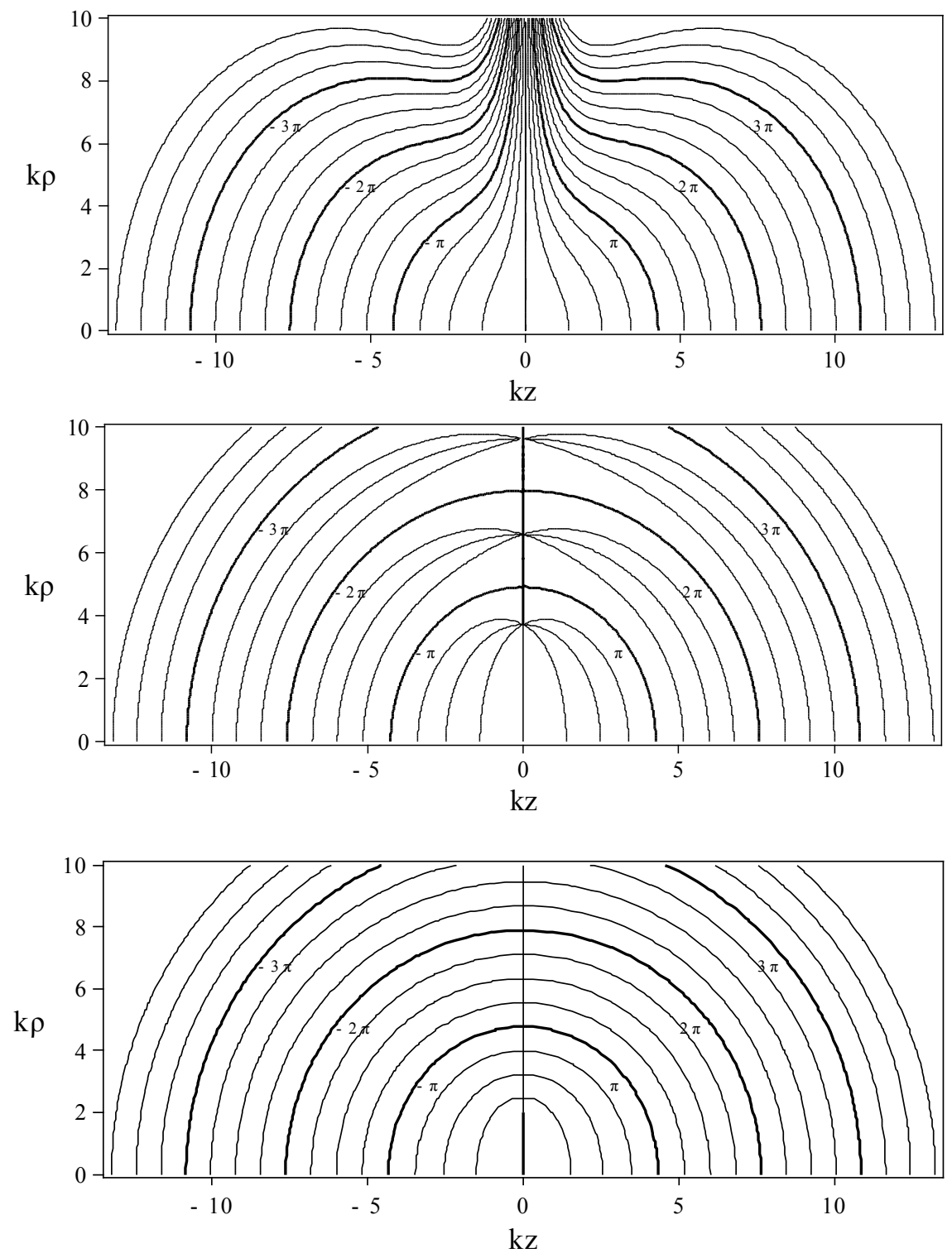

Figure 3.8: Surfaces of constant phase for $\psi_{G}$ (upper figure), $j_{0}(k R)$ (middle figure), and the oblate spheroidal beam $\psi_{01}$ (lower figure), drawn for $\beta=2$ in increments of $\pi / 4$. The darker lines show the isophase surfaces that are integer multiples of $\pi$. The equiphase surfaces for $\psi_{G}$ all go off to infinite $\rho$ in the $z=0$ plane, while those of $j_{0}(k R)$ converge on to the zeros of $j_{0}(k R)$, which lie on the circles $\rho_{\nu}=b\left[(\nu \pi / \beta)^{2}+1\right]^{1 / 2}$. The surfaces with phase equal to an integer multiple of $\pi$ converge on to the circles $\rho=b\left[(X / \beta)^{2}+1\right]^{1 / 2}$, where $\tan (X)=X$. For the oblate spheroidal wave function $\psi_{01}$ the equiphase surfaces are half-ellipsoids $\xi=\xi_{0}, \xi=-\xi_{0}$. These half-ellipsoids tend to hemispheres for large $\xi_{0}^{2}$. 


\section{Normalization integral}

We now examine the convergence of integrals over a cross-section of the beams given by Eqn. 3.83. A physical quantum particle beam, for example, must have finite energy and probability content in a transverse slice of the beam, say between $z$ and $z+\Delta z$. The calculation of such probability or energy content involves an integration of $|\psi|^{2}$ over $\rho$ at fixed $z$. From Eqn. A7 of [16] we have

$$
\int_{0}^{\infty} \rho|\psi|^{2} d \rho=b^{2} \int_{0}^{1} \eta^{-1}\left[\eta^{2}+\left(\frac{z_{0}}{b \eta}\right)^{2}\right]|\psi|^{2} d \eta,
$$

where the integration is over the plane $z=z_{0}$. From Eqns. 3.14 we have $\xi=z_{0} / b \eta$, and as $\eta \rightarrow 0(\xi \rightarrow \infty)$ it follows from Eqn. 3.84 that

$$
\left|\psi_{m n}\right|^{2} \rightarrow(\beta \xi)^{-2}\left[S_{m n}(\beta, \eta)\right]^{2}=\frac{\eta^{2}}{\left(k z_{0}\right)^{2}}\left[S_{m n}(\beta, \eta)\right]^{2} .
$$

Thus if $S_{m n}(\beta, 0)$ is not zero, the leading term in the integrand in Eqn. 3.86 will be $\eta^{-1}$ at small $\eta$ and the integral will diverge logarithmically. We must have $S_{m n}(\beta, 0)=0$ for convergence of the integral. This is for scalar beams, but electromagnetic beams have the same divergences, as shown in [16]. Thus $S_{m n}(\beta, \eta)$ has to be zero at $\eta=0$ and it is known that this happens for odd $n-m$.

The oblate spheroidal wave functions only represent physical beams when $n-m$ is odd. A similar condition holds for the spherical Bessel beams given in Eqn. 3.70, which have convergent normalization integrals only when $n-m$ is odd too. These have a flaw, however, which makes them nonphysical. They contain backward propagating elements which increase in importance for small $\beta$. April $[52,53,54]$ has considered linear combinations of these spherical Bessel solutions, those with $n-m$ odd will have convergent normalization integrals, but the problem of backward propagation remains. Of course, when the physical system includes a mirror or mirrors, there can be backward propagation in some regions of space: see, for example, a paper by Bokor and Davidson [55] on focusing with a paraboloidal mirror. 


\section{Paraxial limit}

We shall now give a comparison of the properties of the oblate spheroidal beam $\psi_{01}$ and the Gaussian beam $\psi_{G}$, in the large $\beta$ (paraxial) limit. For large $\beta$, Flammer [31] has shown that the angular oblate spheroidal functions become linear combinations of Laguerre polynomials. This representation of the angular oblate spheroidal functions also suggests the form of the large $\beta$ representation of the radial oblate spheroidal functions. The radial oblate spheroidal functions, in the large $\beta$ limit, may be expanded in terms of the second solution of the Laguerre differential equation.

Thus, in the case of large $\beta$, we might expect the oblate spheroidal beam $\psi_{01}$ to have similar features to those of the Gaussian beam $\psi_{G}$. A paper by Zeppenfeld [56] has investigated the large $\beta$ limit of the oblate spheroidal wave functions defined in Eqn. 3.82 and given expressions for the radial oblate spheroidal functions in the large $\beta$ limit.

Fig. 3.9 shows the probability densities of a nonparaxial $(\beta=2)$ oblate spheroidal beam, a paraxial Gaussian beam $(\beta=7)$, and also the oblate spheroidal beam with $\beta=7$, on the beam axis. In this figure the modulus of the oblate spheroidal wave function $\psi_{01}$ has been normalized to unity at the origin, i.e. $\left|\psi_{01}(\xi=0, \eta=1)\right|=1$. This corresponds to multiplying the wave function $\psi_{01}$ by the factor $\left[R_{01}^{(2)}(\beta, 0) S_{01}(\beta, 1)\right]^{-1}$.

The paraxial beams $(\beta=7)$ show a similar $z$ dependence, which can be derived explicitly. On the beam axis the probability density of the Gaussian beam is $\beta^{2} /\left[\beta^{2}+(k z)^{2}\right]$, whereas the asymptotic form of the (normalized) oblate spheroidal beam probability density is $1 /\left[R_{01}^{(2)}(\beta, 0)\right]^{2}(k z)^{2}$. For large $k z$, both beams have the same $z$ dependence, but with different amplitudes dependent on $\beta$. However, for large $\beta,-\left[R_{01}^{(2)}(\beta, 0)\right] \approx R_{00}^{(1)}(\beta, 0)$, where

$$
R_{00}^{(1)}(\beta, 0) \approx 2 \sum_{s=0}^{\infty} \sum_{r=0}^{\infty}(-1)^{s} \frac{A_{s}^{00}}{A_{0}^{00}} \frac{(s+r) !(s+r) !}{r ! s !} \frac{1}{(2 \beta)^{s+r+1}},
$$

which gives $R_{00}^{(1)}(\beta, 0) \rightarrow 1 / \beta, \beta \rightarrow \infty$, see p 68 of [31]. Thus along the beam axis, for large $(k z)$, in the limit of large $\beta$, but still small $\beta / k z$, the Gaussian beam probability density is asymptotic to the oblate spheroidal beam probability density. 


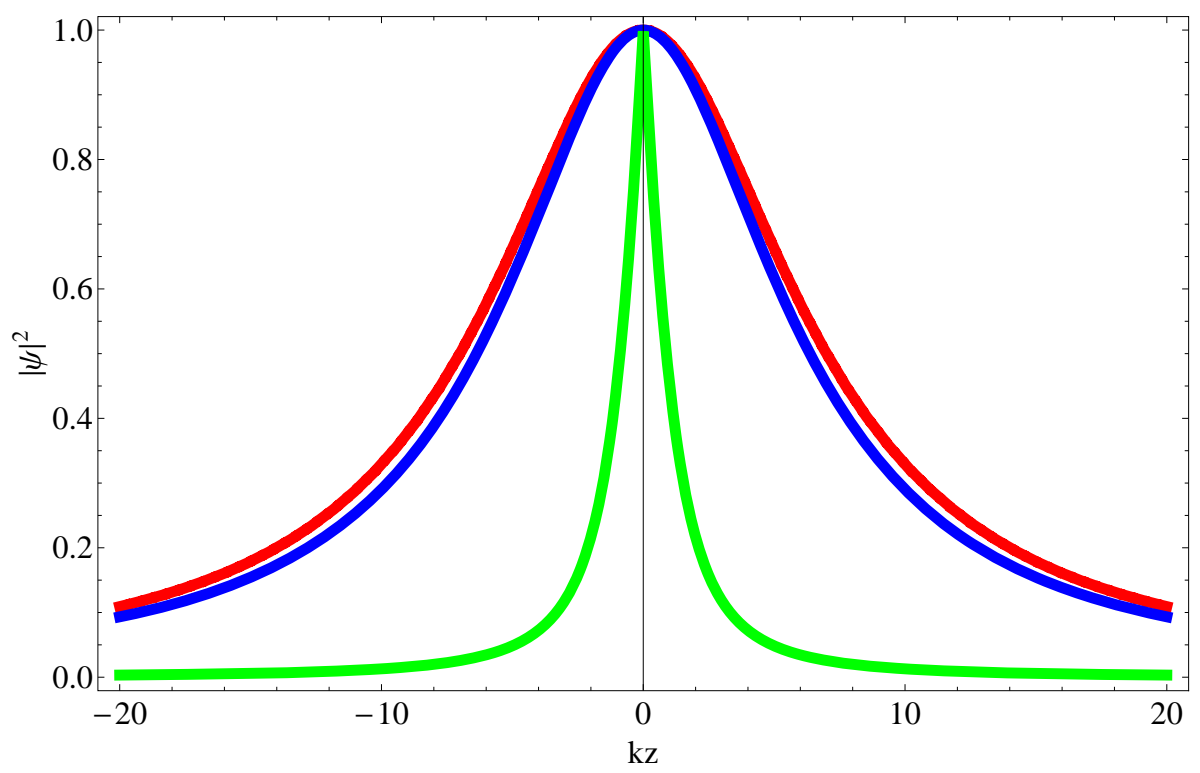

Figure 3.9: Comparison between the (normalized) oblate spheroidal beam $\psi_{01}$ and the Gaussian beam $\psi_{G}$ on the beam axis $(\rho=0)$. In the paraxial case, where $\beta=7$, the Gaussian beam (red) is asymptotic to the (normalized) oblate spheroidal beam $\psi_{01}$ (blue) along the beam axis. The (normalized) oblate spheroidal beam $\psi_{01}$ is also shown in the nonparaxial case, where $\beta=2$ (green). This corresponds to a tightly focused beam.

This result also explains why the confluent Heun beam of Eqn. 3.69 behaves similarly to the Gaussian beam along the beam axis. Since $U(\beta, 1)=$ $\left[R_{00}^{(1)}(\beta, 0)\right]^{-1} \rightarrow \beta$ for large $\beta$, with $\mu(\beta)=\mu_{00}(\beta)$, on the beam axis $(\rho=0)$, $\left|\psi_{H}\right|^{2} \rightarrow \beta^{2} /(k z)^{2}$, for large $k z$.

Now let us consider the large $\beta$ limit of the oblate spheroidal wave function $\psi_{01}(\xi, \eta)$ in the focal plane. The normalized oblate spheroidal wave function is $\psi_{01}(\xi, \eta)=i R_{01}^{(3)}(\beta, \xi) S_{01}(\beta, \eta) /\left[R_{01}^{(2)}(\beta, 0) S_{01}(\beta, 1)\right]$. This ensures the probability density is unity at the origin $(\rho=z=0)$. In the focal plane $(z=0)$, for $\rho \geq b, S_{01}(\beta, \eta)=0$ and thus $\psi_{01}(\xi, \eta=0)=0$. When $z=0$, for $\rho \leq b$, the oblate spheroidal beam is $\psi_{01}(\xi=0, \eta)=i S_{01}(\beta, \eta) / S_{01}(\beta, 1)$. 
In the large $\beta$ limit the angular oblate spheroidal functions are (p 63 [31]):

$$
\begin{aligned}
\bar{S}_{m n}(\beta, \eta) & \approx\left(1-\eta^{2}\right)^{m / 2} \sum_{s=-\nu}^{\infty} A_{s}^{m n}\left\{e^{-\beta(1-\eta)} L_{\nu+s}^{(m)}[2 \beta(1-\eta)]\right. \\
& \left.+(-1)^{n-m} e^{-\beta(1+\eta)} L_{\nu+s}^{(m)}[2 \beta(1+\eta)]\right\}
\end{aligned}
$$

The bar is used to indicate that this holds only in the large $\beta$ limit. In this expression, for $n-m$ odd, $\nu=\frac{1}{2}(n-m-1)$, and the functions $L_{\nu+s}^{(m)}(x)$ denote the Laguerre polynomials, see Chapter 22 of [30] for properties of these functions. The coefficients $A_{ \pm r}^{m n}$ are known, up to a constant $A_{0}^{m n}$, and expansions of these functions are given in Chapter 8 of [31]:

$$
\frac{A_{ \pm r}^{m n}}{A_{0}^{m n}}=\sum_{k=r}^{\infty} a_{k}^{ \pm r}(m, n) \beta^{-k}
$$

Now for $n=1, m=0$, we have, as $\beta \rightarrow \infty(\mathrm{p} 62[31])$ :

$$
\left[\frac{S_{01}(\beta, \eta)}{S_{01}(\beta, 1)}\right] \rightarrow e^{-\beta(1-\eta)} L_{0}[2 \beta(1-\eta)], \quad 0<\eta \leq 1 .
$$

The first few Laguerre polynomials are $L_{0}(x)=1, L_{1}(x)=-x+1$, etc. Therefore when $z=0$, for $\rho \leq b$, we have the following

$$
\left|\psi_{01}(\xi=0, \eta)\right|^{2}=\left[\frac{S_{01}(\beta, \eta)}{S_{01}(\beta, 1)}\right]^{2} \rightarrow e^{-2 \beta(1-\eta)}, \quad \beta \rightarrow \infty .
$$

In the region $\xi=0$ we have $\eta^{2}=1-(k \rho / \beta)^{2}$. We have already shown, in Eqn. 3.75, that when $z=0$ the Gaussian beam is a good approximation to a scalar beam if $k \rho<<\beta$ and $\beta^{2}>>1$. If we assume $k \rho<<\beta$, then the expression for $\eta$ becomes $\eta=1-\frac{1}{2}(k \rho / \beta)^{2}+O\left((k \rho / \beta)^{4}\right)$. Substituting this into Eqn. 3.92 we have, for $k \rho<<\beta$ and $\beta^{2}>>1$,

$$
\left|\psi_{01}(\xi=0, \eta)\right|^{2}=\left[\frac{S_{01}(\beta, \eta)}{S_{01}(\beta, 1)}\right]^{2} \rightarrow \exp \left(-(k \rho)^{2} / \beta\right) .
$$


The Gaussian beam probability density is given by $\exp \left(-(k \rho)^{2} / \beta\right)$ in the focal plane. Therefore, we have proved that, in the large $\beta$ limit, for $z=0$ and $k \rho<<\beta$, the oblate spheroidal beam $\psi_{01}(\xi, \eta)$ has a leading term with similar properties as the Gaussian beam. In the region $\eta=0, \psi_{01}(\xi, \eta)=0$, whereas the Gaussian beam has $\left|\psi_{G}\right|^{2}=\exp \left(-(k \rho)^{2} / \beta\right)$. In the next section we shall show that the oblate spheroidal wave functions $\psi_{m n}(\xi, \eta)$, with $n-m$ odd, exhibit nonphysical behaviour in the focal plane $z=0$, and consequently that they are not the nonparaxial generalization of the Laguerre-Gaussian beams. Thus the analysis of this section shows that, when $\beta \rightarrow \infty$, the oblate spheroidal beam $\psi_{01}(\xi, \eta)$ has some similar properties as the Gaussian beam, but in this limit it is simpler to use the Gaussian beam expression. The simplest of the possible scalar oblate spheroidal beams, i.e. $n=1, m=0$, is shown in Fig. 3.10

For comparison, we shall briefly discuss the large $\beta$ limit of the spherical Bessel beams. Along the beam axis, for large $\beta$, the probability density of the (normalized) spherical Bessel beam $\psi_{00}$ is asymptotic to the probability density of the Gaussian beam. However, in the large $\beta$ limit, the spherical Bessel beams as defined do not reduce to the paraxial Gaussian beam expression. April $[52,53,54]$ has shown that certain linear combinations of the spherical Bessel beams are needed to produce exact beam wave functions which reduce to the Laguerre-Gaussian beams in the paraxial limit (of which $\psi_{G}$ is the first member.) 


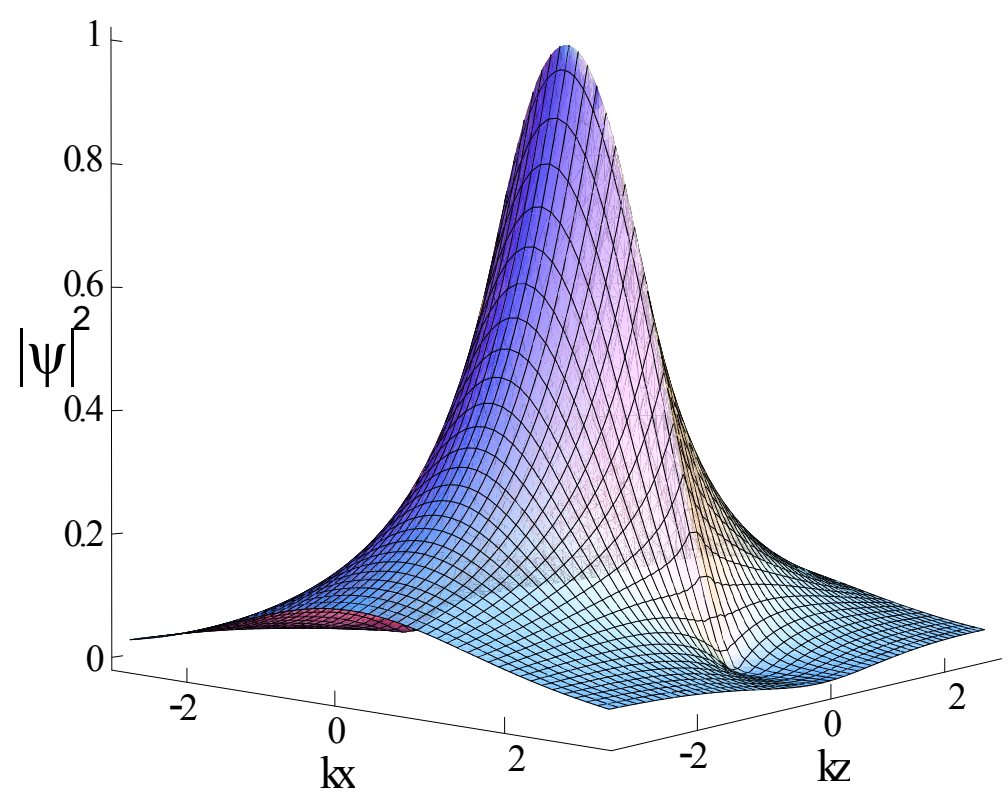

Figure 3.10: The modulus squared of the (normalized) oblate spheroidal wave function $\psi_{01}(\xi, \eta)=i R_{01}^{(3)}(\beta, \xi) S_{01}(\beta, \eta)$ in the $y=0$ plane, plotted for a tightly focused beam with $\beta=2$. The $z$ axis defines the propagation direction of the beam, and $\rho=\sqrt{x^{2}+y^{2}}$ is the radial distance from the beam axis. Note that the solution is identically zero outside the circle $\rho=b$ in the focal plane $z=0$.

\section{Oblate spheroidal beam problems}

The oblate spheroidal wave functions $\psi_{m n}(\xi, \eta)$ can only represent physical scalar beams when $n-m$ is odd. However, this subset exhibits nonphysical behaviour in the focal plane, both inside and outside the circle $\rho=b$. Fig. 3.10 shows that, in the region $z=0, \rho \leq b$, the $z$ derivative of the probability density is discontinuous. This occurs for all oblate spheroidal beams with $n-m$ odd, which we shall now demonstrate analytically.

The $z$ derivative of a wave function $\psi$ is given by [16]:

$$
\frac{\partial \psi}{\partial z}=\frac{1}{b\left(\xi^{2}+\eta^{2}\right)}\left[\eta\left(1+\xi^{2}\right) \frac{\partial \psi}{\partial \xi}+\xi\left(1-\eta^{2}\right) \frac{\partial \psi}{\partial \eta}\right] .
$$

Now consider the $z$ derivative of Eqn. 3.83 (with $n-m$ odd),

$$
\begin{array}{r}
\frac{\partial \psi}{\partial z}=\frac{i e^{i m \phi}}{b\left(\xi^{2}+\eta^{2}\right)}\left[\eta\left(1+\xi^{2}\right) S_{m n}(\beta, \eta) \frac{d}{d \xi} R_{m n}^{(3)}(\beta, \xi)\right. \\
\left.+\xi\left(1-\eta^{2}\right) R_{m n}^{(3)}(\beta, \xi) \frac{d}{d \eta} S_{m n}(\beta, \eta)\right]
\end{array}
$$


Taking the limit as $\xi \rightarrow 0$ we find

$$
\begin{gathered}
\left.\frac{\partial \psi}{\partial z}\right|_{\xi \rightarrow 0}=\lim _{\xi \rightarrow 0} \frac{i S_{m n}(\beta, \eta)}{b \eta} e^{i m \phi}\left[\frac{d}{d \xi} R_{m n}^{(1)}(\beta, \xi)+i \frac{d}{d \xi} R_{m n}^{(2)}(\beta, \xi)\right] . \\
\left.\Rightarrow \frac{\partial \psi}{\partial z}\right|_{\xi=0}:=\left\{\begin{array}{c}
\frac{i S_{m n}(\beta, \eta)}{b \eta} e^{i m \phi}\left[R_{m n}^{(1)^{\prime}}(\beta, 0)+i R_{m n}^{(2)^{\prime}}\left(\beta, 0^{+}\right)\right], \xi \rightarrow 0^{+}, \\
\frac{i S_{m n}(\beta, \eta)}{b \eta} e^{i m \phi}\left[R_{m n}^{(1)^{\prime}}(\beta, 0)+i R_{m n}^{(2)^{\prime}}\left(\beta, 0^{-}\right)\right], \xi \rightarrow 0^{-} .
\end{array}\right.
\end{gathered}
$$

The prime denotes differentiation with respect to $\xi$. The first function in the square brackets of Eqn. 3.97 is continuous, as $\xi \rightarrow 0$, from both sides. The second function is discontinuous, having different limits as $\xi \rightarrow 0^{+}$and $\xi \rightarrow 0^{-}$. The function $R_{m n}^{(2)}(\beta, \xi)$ is even when $n-m$ is odd, however, its derivative is discontinuous, at $\xi=0$, which leads to the (nonphysical) bladelike behaviour observed in the plot of $|\psi|^{2}$ in Fig. 3.10. The discontinuity in $R_{01}^{(2)^{\prime}}(\beta, 0)$ is evident in Fig. 3.11, which shows $R_{01}^{(2)}(\beta, \xi)$ and its derivative, plotted for $\beta=2$. The discontinuity is apparent for all oblate spheroidal beams with $n-m$ odd, and also means that the radial equation Eqn. 3.76 is not satisfied inside the disk $z=0, \rho \leq b$.

When $\eta=0$, the oblate spheroidal wave functions with $n-m$ odd are identically zero. However, these wave functions also have a discontinuous $z$ derivative in this region. The $z$ derivative of Eqn. 3.83 (with $n-m$ odd), for $\eta=0$, is

$$
\begin{gathered}
\left.\frac{\partial \psi}{\partial z}\right|_{\eta=0}=\frac{i R_{m n}^{(3)}(\beta, \xi)}{b \xi} e^{i m \phi} S_{m n}^{\prime}(\beta, 0) . \\
\left.\Rightarrow \frac{\partial \psi}{\partial z}\right|_{\eta=0}:=\left\{\begin{array}{c}
\frac{i S_{m n}^{\prime}(\beta, 0) e^{i m \phi}}{b \xi_{0}}\left[R_{m n}^{(1)}\left(\beta, \xi_{0}\right)+i R_{m n}^{(2)}\left(\beta, \xi_{0}\right)\right], \xi=\xi_{0}, \\
\frac{i S_{m n}^{\prime}(\beta, 0) e^{i m \phi}}{b \xi_{0}}\left[R_{m n}^{(1)}\left(\beta, \xi_{0}\right)-i R_{m n}^{(2)}\left(\beta, \xi_{0}\right)\right], \xi=-\xi_{0} .
\end{array}\right.
\end{gathered}
$$

The $z$ derivative given in Eqn. 3.99 is discontinuous, at $z=0$, for $\rho \geq b$. Furthermore, it is tending to an infinite value at the point $\xi=\eta=0$.

The oblate spheroidal wave functions with $n-m$ odd have a discontinuous $z$ derivative, at $z=0$, both inside and outside the circle $\rho=b$. The discontinuous $z$ derivative of the oblate spheroidal beams, with $n-m$ odd, is nonphysical. 


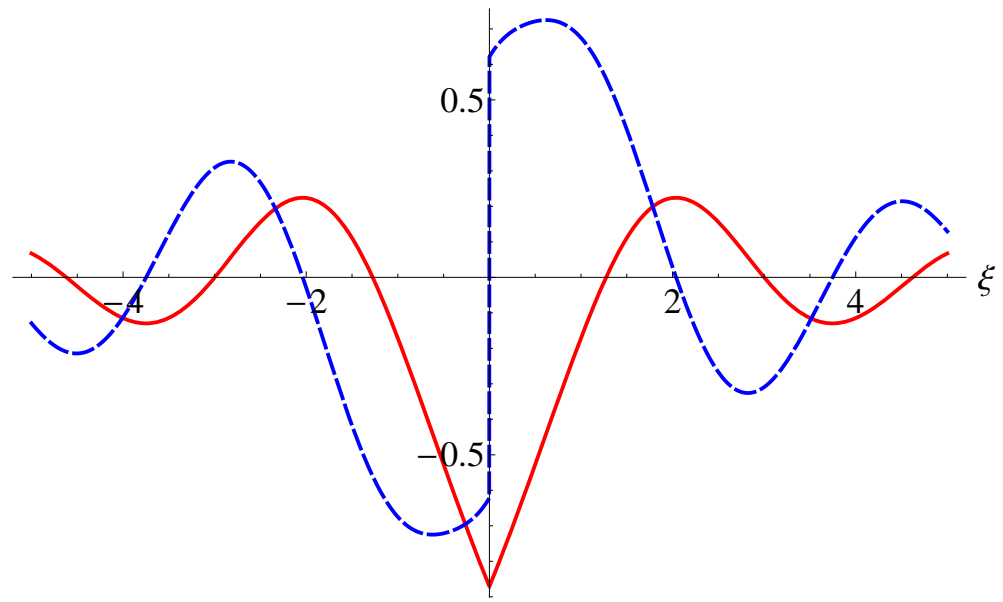

Figure 3.11: The radial oblate spheroidal function $R_{01}^{(2)}(\beta, \xi)$ (red-line) and its derivative (blue-dashed), plotted for $\beta=2$. The function $R_{01}^{(2)^{\prime}}(\beta, \xi)$ is discontinuous, at $\xi=0$, with opposite limits as $\xi \rightarrow 0^{+}$and $\xi \rightarrow 0^{-}$.

An example of a wave function with a discontinuous derivative is the wellknown case of a delta function potential, see Chapter 2 of [57]. However, a free space scalar beam should have finite and continuous derivatives at all regions of space.

In conclusion, the oblate spheroidal wave functions proposed in [17] have been shown to be possible representations of physical beams only when the angular function $S_{m n}(\beta, \eta)$ has odd $n-m$. This condition makes $S_{m n}(\beta, \eta)$ odd in $\eta$, which ensures the convergence of integrals of physical quantities over a cross-section of the beam. The odd $n-m$ condition also makes $\psi_{m n}(\xi, \eta)$ zero in the focal plane $z=0$ outside the circle $\rho=b$, and thus allows for the physically necessary discontinuity in phase at $z=0$ on the ellipsoidal surfaces of otherwise constant phase.

However, the necessary odd $n-m$ condition leads to nonphysical behaviour of the beam in the focal plane $z=0$. The oblate spheroidal wave functions with $n-m$ odd have a discontinuous $z$ derivative, at $z=0$, both inside and outside the circle $\rho=b$. This behaviour is nonphysical, since a free space scalar beam must have finite and continuous derivatives at all regions of space. The oblate spheroidal wave functions are not physical scalar beams, and are not the nonparaxial generalization of the Gaussian beam, in their current simple product form that is. 


\section{Confluent Heun and oblate spheroidal relations}

Section 3.6.1 considered the separable solutions of the Helmholtz equation in terms of confluent Heun functions, which are not physically realizable. This section has shown the oblate spheroidal wave functions are also not physically realizable. Both sets of functions can be related. Comparison of the asymptotic expression in Eqn. 3.79 for the radial oblate spheroidal functions with the asymptotic expressions for the radial confluent Heun functions in Eqn. 3.47 and Eqn. 3.49 shows that the relation between these functions is:

$$
\begin{aligned}
& R_{0 n}^{(1)}(\beta, \xi)=\frac{\sin \left[\frac{\pi}{2}(n+1)\right]}{U(\beta, 1)} X_{0}^{\text {even }}(\xi), \quad n \text { even }, \\
& R_{0 n}^{(1)}(\beta, \xi)=\frac{\cos \left[\frac{\pi}{2}(n+1)\right]}{V(\beta, 1)} X_{0}^{\text {odd }}(\xi), \quad n \text { odd } .
\end{aligned}
$$

In the case of the angular oblate spheroidal functions and the angular confluent Heun functions the relation is

$$
\begin{aligned}
& S_{0 n}^{(1)}(\beta, \eta)=Y_{0}^{\text {even }}(\eta) P_{n}(0), \quad n \text { even } \\
& S_{0 n}^{(1)}(\beta, \eta)=Y_{0}^{\text {odd }}(\eta) P_{n}^{\prime}(0), \quad n \text { odd. }
\end{aligned}
$$

The angular oblate spheroidal functions are defined using the scheme given in [30] and [31], and $P_{n}(0)$ denotes the $n$th Legendre polynomial of the first kind. These relations assume $\mu=\mu_{0 n}(\beta), n=0,2,4, \ldots$ for the even functions, whereas $\mu=\mu_{0 n}(\beta), n=1,3,5, \ldots$ for the odd functions.

\subsubsection{Non-existence of separable spheroidal beams}

Section 3.6.2 showed that none of the oblate spheroidal wave functions represent physical free space scalar beams. A physical free space scalar beam must have finite derivatives, at all regions of space, and they must also be continuous. In this section we shall use these constraints to show that, in general, there is no separable solution of the Helmholtz equation, in oblate spheroidal coordinates, which has these properties.

We make the following demands on a physical wave function $\psi$ : 
1. $\psi$ must satisfy the Helmholtz equation Eqn. 3.3. Note that this implies that the derivatives $\partial_{z} \psi$ and $\partial_{\rho} \psi$ exist and are continuous: a discontinuous first derivative would imply an infinite second derivative, with nothing in the free space Helmholtz equation to cancel it.

2. The integral $\int_{0}^{\infty} \rho|\psi|^{2} d \rho$ must be finite. For example, $2 \pi \Delta z$ times this integral represents the probability of finding a particle in a transverse section of thickness $\Delta z$ in a Schrödinger particle beam.

3. The $z$-component of the probability current density $J_{z}$, proportional to $\operatorname{Im}\left(\psi^{*} \partial_{z} \psi\right)$, must be non-zero, at least in the central part of the focal plane, otherwise the wave function would not represent a propagating beam.

We could also require the finiteness of the total flux, i.e. of the integral $\int_{0}^{\infty} \rho J_{z} d \rho$, but shall not need to do so.

The oblate spheroidal wave functions given in Eqn. 3.83, with $n-m$ odd, were shown in section 3.6.2 to satisfy condition (2), and also to have physically realizable isophase surfaces. However, we also proved in that section that the $z$ derivative of these wave functions is discontinuous in the focal plane, which violates condition (1). As has already been stated, the oblate spheroidal wave functions are not physical scalar beams. The remainder of this section shall prove a more general result, independent of the properties of the oblate spheroidal functions.

Let the solution to Helmholtz's equation be represented as

$$
\psi(\xi, \eta, \phi)=R(\xi) S(\eta) \Phi(\phi)
$$

The functions $R(\xi), S(\eta)$, need not be the oblate spheroidal functions, but are arbitrary functions which solve the ordinary differential equations in Eqn. 3.76 and Eqn. 3.81. In what follows we shall prove the following theorem

Theorem 2. Free space scalar beams cannot be represented as $\psi(\xi, \eta, \phi)=$ $R(\xi) S(\eta) \Phi(\phi)$ in oblate spheroidal coordinates. 
Proof: Consider the $z$ derivative, defined in Eqn. 3.94, of the beam wave function in Eqn. 3.104:

$$
\begin{aligned}
& \left.\frac{\partial \psi}{\partial z}\right|_{\eta=0}=\frac{1}{b \xi} R(\xi) S^{\prime}(0) \Phi(\phi), \\
& \left.\frac{\partial \psi}{\partial z}\right|_{\xi=0}=\frac{1}{b \eta} R^{\prime}(0) S(\eta) \Phi(\phi) .
\end{aligned}
$$

The $z$ derivative in Eqn. 3.105 must be finite as $\xi \rightarrow 0$, and the $z$ derivative in Eqn. 3.106 must be finite as $\eta \rightarrow 0$. Thus the necessary (but not sufficient) conditions for the finiteness of $\partial_{z} \psi$ on the circle $\xi=\eta=0$ are

$$
R^{\prime}(0) S(0)=0 \quad \text { and } \quad R(0) S^{\prime}(0)=0 .
$$

These conditions will be satisfied if one or more of the following four constraints are satisfied

$$
\left\{\begin{array}{llll}
(a) & S(0)=0 & \text { and } & R(0)=0 \\
(b) & R^{\prime}(0)=0 & \text { and } & S^{\prime}(0)=0 \\
(c) & R^{\prime}(0)=0 & \text { and } & R(0)=0 \\
(d) & S(0)=0 & \text { and } & S^{\prime}(0)=0 .
\end{array}\right.
$$

If (a) holds then $\psi(\rho, z=0)=0 \Rightarrow J_{z}(\rho, z=0)=0$, which gives no flux in the entire focal plane, a condition contrary to (3). Case (b) gives $\left.\partial_{z} \psi\right|_{z=0}=0 \Rightarrow J_{z}(\rho, z=0)=0$, and also implies the nonphysical result of zero flux in the focal plane, which is contrary to condition (3). For case (c), $\psi=0$ in the region $z=0, \rho \leq b$, and would mean there is no flux through the central disk of the focal plane, which is nonphysical (consider paraxial beams). If (d) holds then both $\psi$ and $\partial_{z} \psi$ will be zero for $\eta=0$, i.e. for $z=0, \rho \geq b$, which is not a fatal flaw. But if $S(0)$ and $S^{\prime}(0)$ are both zero, the series expansion of $S(\eta)$ would start with the second or higher power of $\eta$, which is contrary to the indicial equation of Eqn. 3.81, which gives the characteristic exponents 0 and 1 . Thus, in all possible cases, it is not possible to have a wave function, in the form of Eqn. 3.104, which represents a physical scalar beam. QED 


\subsection{Oblate spheroidal electromagnetic beams}

For tightly focused beams the vector nature of light must be taken into account. That is, given the scalar solutions of Helmholtz's equation, we must derive the vector components of the electric and magnetic fields. We are again interested in the free space properties of such electromagnetic fields and it is this situation which we shall confine our attention to. The method of constructing solutions of the Maxwell equations from solutions of the Helmholtz equation may be found in [16] or [43], which we shall summarize.

The oblate spheroidal wave functions $\psi_{m n}(\xi, \eta)$ are exact solutions of the Helmholtz equation. For $n-m$ odd such solutions are forward propagating in the far field, allow for the discontinuity in phase at $z=0$ on the isophase surfaces, and are normalizable in any cross-section of the beam. However, these beam solutions have a discontinuous $z$ derivative, in the entire $z=0$ plane. We shall investigate electromagnetic beams based on these scalar wave functions and exhibit nonphysical behaviour in the electromagnetic case also.

The solutions of the Helmholtz equation, in terms of the oblate spheroidal wave functions, are

$$
\psi_{m n}(\xi, \eta)=i\left[R_{m n}^{(1)}(\beta, \xi)+i R_{m n}^{(2)}(\beta, \xi)\right] S_{m n}(\beta, \eta) e^{i m \phi}
$$

where $n-m$ is odd. Examples of electromagnetic beams that can be constructed from the scalar beam solutions are transverse electric (TE) or transverse magnetic (TM) beams. In a TE beam the $z$ component of the electric field is zero, while in a TM beam the $z$ component of the magnetic field is zero. Following the notation in [16] we shall use Gaussian units for the electric and magnetic fields. (The fields $\mathbf{E}(\mathbf{r})$ and $\mathbf{B}(\mathbf{r})$ in SI units correspond to the fields $\mathbf{E}(\mathbf{r}) / \sqrt{4 \pi \epsilon_{0}}$ and $\sqrt{\mu_{0} / 4 \pi} \mathbf{B}(\mathbf{r})$ in Gaussian units.)

In the case of a TE mode, the electric and magnetic fields may be deduced from the TM modes (and vice versa) by means of the duality transformations $\mathbf{E}(\mathbf{r}) \rightarrow \mathbf{B}(\mathbf{r}), \mathbf{B}(\mathbf{r}) \rightarrow-\mathbf{E}(\mathbf{r})$. For a TM beam the magnetic vector potential has the simple form $\mathbf{A}_{T M}(\mathbf{r})=\left[0,0, A_{0} \psi(\mathbf{r})\right]$, where $\psi(\mathbf{r})$ is the solution of the scalar Helmholtz equation given by Eqn. 3.109, and $A_{0}$ is a constant. The vector potential $\mathbf{A}_{T E}$, which gives the dual of the TM field arising from $\mathbf{A}_{T M}$, is $\mathbf{A}_{T E}=(i k)^{-1} \nabla \times \mathbf{A}_{T M}$. Further details may be found in [16]. 
The magnetic vector potential for a TE mode is

$$
\mathbf{A}_{T E}=\frac{A_{0}}{i k}\left(\frac{\partial \psi}{\partial y},-\frac{\partial \psi}{\partial x}, 0\right) .
$$

The corresponding electric and magnetic fields are

$$
\begin{aligned}
& \mathbf{E}(\mathbf{r})=A_{0}\left(\frac{\partial \psi}{\partial y},-\frac{\partial \psi}{\partial x}, 0\right), \\
& \mathbf{B}(\mathbf{r})=\frac{A_{0}}{i k}\left(\frac{\partial^{2} \psi}{\partial x \partial z}, \frac{\partial^{2} \psi}{\partial y \partial z}, \frac{\partial^{2} \psi}{\partial z^{2}}+k^{2} \psi\right) .
\end{aligned}
$$

The electric field in Eqn. 3.111 is purely transverse, and if $\psi$ is axially symmetric (no azimuthal dependence) the electric field lines are circles concentric with the $z$ axis. An axially symmetric $\psi$ also leads to a TE beam within which the electric field is exactly linearly polarized everywhere [50]. Thus exact vector electromagnetic beams which solve Maxwell's equations can be constructed by taking various derivatives of the solutions of the scalar Helmholtz equation.

However, there is a problem with the TE or TM vector beams constructed from the scalar solutions given in Eqn. 3.109. That is, for odd $n-m$, the resulting electric or magnetic field are divergent in the focal plane at the point $\xi=\eta=0$.

We shall investigate a TE beam in the following paragraphs. The electric field is given in Eqn. 3.111. In oblate spheroidal coordinates the $\rho$ derivative of $\psi$ is:

$$
\frac{\partial \psi}{\partial \rho}=\frac{\left(\xi^{2}+1\right)^{1 / 2}\left(1-\eta^{2}\right)^{1 / 2}}{b\left(\xi^{2}+\eta^{2}\right)}\left\{\xi \frac{\partial \psi}{\partial \xi}-\eta \frac{\partial \psi}{\partial \eta}\right\} .
$$

The $x$ and $y$ derivatives are related to the derivatives with respect to $\rho$ and $\phi$ by the following formulae:

$$
\begin{aligned}
& \frac{\partial \psi}{\partial x}=\cos \phi \frac{\partial \psi}{\partial \rho}-\frac{\sin \phi}{\rho} \frac{\partial \psi}{\partial \phi}, \\
& \frac{\partial \psi}{\partial y}=\sin \phi \frac{\partial \psi}{\partial \rho}+\frac{\cos \phi}{\rho} \frac{\partial \psi}{\partial \phi}
\end{aligned}
$$

where $\phi$ is the azimuthal angle. In subsequent paragraphs $\psi$ shall be given by the expression in Eqn. 3.109, which gives $\partial_{\phi} \psi=i m \psi$. Now suppose we consider $\frac{\partial \psi}{\partial \rho}$ in the focal plane $(z=0)$ with $\rho \geq b$ initially. 
In oblate spheroidal coordinates this corresponds to $\eta=0$. (Since $\psi=0$ when $\eta=0$, the $\phi$ derivative does not contribute to the electric field, only the $\rho$ derivative does.) The $\rho$ derivative is

$$
\begin{aligned}
\left.\frac{\partial \psi}{\partial \rho}\right|_{\eta=0} & =\frac{i\left(\xi^{2}+1\right)^{1 / 2}}{b \xi} S_{m n}(\beta, 0) e^{i m \phi} \frac{d}{d \xi} R_{m n}^{(3)}(\beta, \xi), \\
& =0, \quad \forall \xi \neq 0 .
\end{aligned}
$$

This is due to the fact that, for odd $n-m, S_{m n}(\beta, \eta)$ is zero when $\eta=0$. Eqn. 3.116 shows that the electric field of a TE beam is zero, in the focal plane $z=0$, outside the circle $\rho=b$. This result arises from forcing the scalar wave function to be zero in this region. It is interesting to consider what experimental implications such an electromagnetic beam would produce. In other words, is it possible to create a free space (TE) electromagnetic beam that is zero, identically, in the focal plane $z=0$, outside the circle $\rho=b$ ?

Let us now consider the limit from the other side of the circle $\rho=b$. That is, in the focal plane $z=0$, but now with $\xi=0$ and $\eta \rightarrow 0$. In this case we find

$$
\begin{aligned}
\left.\frac{\partial \psi}{\partial \rho}\right|_{\xi=0} & =\frac{-i\left(1-\eta^{2}\right)^{1 / 2}}{b \eta} R_{m n}^{(3)}(\beta, 0) e^{i m \phi} \frac{d}{d \eta} S_{m n}(\beta, \eta), \\
& =\frac{\left(1-\eta^{2}\right)^{1 / 2}}{b \eta} R_{m n}^{(2)}(\beta, 0) e^{i m \phi} \frac{d}{d \eta} S_{m n}(\beta, \eta) .
\end{aligned}
$$

If we take the limit as $\eta \rightarrow 0$

$$
\begin{aligned}
\left.\frac{\partial \psi}{\partial \rho}\right|_{\xi=0, \eta \rightarrow 0} & =\lim _{\eta \rightarrow 0} \frac{R_{m n}^{(2)}(\beta, 0)}{b \eta} e^{i m \phi} \frac{d}{d \eta} S_{m n}(\beta, \eta), \\
& =\lim _{\eta \rightarrow 0} \frac{(-1)^{\frac{n-m-1}{2}}(n+m+1) !}{2^{n}\left(\frac{n-m-1}{2}\right) !\left(\frac{n+m+1}{2}\right) !} \frac{R_{m n}^{(2)}(\beta, 0)}{b \eta} e^{i m \phi} .
\end{aligned}
$$

The Flammer normalization of the derivative of $S_{m n}(\beta, \eta)$ with $n-m$ odd has been used in Eqn. 3.118 [30]. The normalization is irrelevant in any case; the Meixner-Schäfke, or Stratton-Morse-Chu-Little-Corbatò schemes could have been chosen. The most important feature is that the electric field (and hence the magnetic field in the TM beam case) is divergent. 
The electric field in the region $z=0, \rho<b$, diverges as we approach the circle $z=0, \rho=b$, as $1 / \eta$. (The contribution to the electric field from the $\phi$ derivative is finite, and cannot 'cancel' the divergence.)

A divergent electric field at the point $\xi=\eta=0$ is allowed in the case of diffraction by a circular disk, provided the energy density is integrable. Large fields are expected at the edge of certain obstacles. In the case of diffraction by a circular disk, Flammer $[58,59]$ has given the form of the field components, near the disk, and also discussed their singular behaviour. The nature of the electric fields and charge densities at corners and edges can be found in Chapter 2 of Jackson [43].

The solution considered in this section is, however, concerned with free space propagation. The question of edge conditions and allowable singularities does not arise. From a physical point of view, since space is isotropic and homogeneous, there should be no divergence associated with a physical free space electromagnetic beam. This analysis shows that the oblate spheroidal wave functions cannot represent physical free space (TE and TM) vector beam solutions.

We may perform the same analysis for the simplest of the spherical Bessel solutions of the Helmholtz equation,

$$
j_{0}(k R)=\frac{\sin (k R)}{k R}, \quad R=b(\xi-i \eta) .
$$

In the limit that $\xi \rightarrow 0, \eta \rightarrow 0$, the $\rho$ derivative of $j_{0}$ has the form

$$
\begin{aligned}
& \left.\frac{\partial}{\partial \rho} j_{0}(k R)\right|_{\eta=0, \xi \rightarrow 0}=\frac{-1}{3} \frac{\beta^{2}}{b}+\frac{1}{30} \frac{\beta^{2}\left(\beta^{2}-5\right)}{b} \xi^{2}+O\left(\xi^{4}\right) . \\
& \left.\frac{\partial}{\partial \rho} j_{0}(k R)\right|_{\xi=0, \eta \rightarrow 0}=\frac{-1}{3} \frac{\beta^{2}}{b}+\frac{1}{30} \frac{\beta^{2}\left(5-\beta^{2}\right)}{b} \eta^{2}+O\left(\eta^{4}\right) .
\end{aligned}
$$

The simplest of the spherical Bessel beams has a finite derivative at the point $\xi=\eta=0$. Furthermore, the derivative has the same limiting value, regardless of which side of the circle $z=0, \rho=b$ the limit is taken from. This is in contrast to the oblate spheroidal beam solution which has a discontinuous derivative across the boundary $\xi=0, \eta=0$. 
We have shown that the oblate spheroidal wave functions cannot represent physical TE and TM electromagnetic beams propagating in free space. The problem with these wave functions arises from their behaviour when $\xi=\eta=0$. In the case of scattering by a disk, the infinite electric field amplitude is allowed due to edge conditions. The energy density remains integrable and a finite total cross-section also results [58, 59]. A free space vector beam, however, should not have associated divergences. The problem therefore remains to determine exact solutions of Maxwell's equations for electromagnetic beams propagating in free space.

\subsection{Bessel beams and the Bateman solution}

\subsubsection{Generalized Bessel beams}

Exact solutions of the Helmholtz equation can be expressed as oblate spheroidal wave functions. None of the oblate spheroidal wave functions are physical free space scalar beams, as shown in section 3.6.2. We shall briefly investigate another set of scalar beam solutions, which have very interesting properties and are physical solutions of the Helmholtz equation. These beams are known as generalized Bessel beams.

The Helmholtz equation in cylindrical polar coordinates is given by

$$
\left(\partial_{\rho}^{2}+\frac{1}{\rho} \partial_{\rho}+\partial_{z}^{2}+\frac{1}{\rho^{2}} \partial_{\phi}^{2}+k^{2}\right) \psi(\rho, z, \phi)=0
$$

where $k$ is the angular wavenumber. The Helmholtz equation is separable in cylindrical polar coordinates and so we may express the wave function as $\psi(\rho, z, \phi)=X(\rho) Z(z) \Phi(\phi)$. Inserting this product into Eqn. 3.122 gives three second-order ordinary differential equations. Solving these three differential equations gives the following product solutions of the Helmholtz equation

$$
\psi_{m}(\rho, z, \phi)=e^{i q z} J_{m}(\kappa \rho) e^{i m \phi}, \quad k^{2}=\kappa^{2}+q^{2} .
$$

In this notation $J_{m}$ is the $m$ th order Bessel function of the first kind and $m$ is an integer, see Chapter 9 of [30] for properties of Bessel functions. 
Barnett and Allen [60] used the separable solutions of Eqn. 3.123 to construct a light beam in which the electric field has the form

$$
\begin{aligned}
\mathbf{E}(\rho, z, \phi) & =\int_{0}^{k} f(\kappa)\left\{(\alpha \hat{\mathbf{x}}+\beta \hat{\mathbf{y}}) F_{m}\right. \\
& \left.+\frac{\kappa}{2 q} \hat{\mathbf{z}}\left[(i \alpha-\beta) F_{m-1}-(i \alpha+\beta) F_{m+1}\right]\right\} d \kappa .
\end{aligned}
$$

Here the shorthand notation $F_{m}=e^{i q z} J_{m}(\kappa \rho) e^{i m \phi}$ has been used.

Barnett and Allen considered monochromatic beams defined through Eqn. 3.124. Using this representation for the electric field they found expressions for the energy per unit length, and for the linear and angular momentum per unit length. They found that all three quantities were invariants, that is, independent of the longitudinal coordinate $z$.

For a monochromatic electromagnetic beam it has been shown by Lekner [61] that of these three quantities only the momentum content per unit length is an invariant in general. There are seven universal invariants associated with certain integrals of a flux density over a section of the beam, but neither the energy content per unit length, or the angular momentum content per unit length are, in general, an invariant.

The proof of this result is based on conservation laws, i.e. conservation of energy, conservation of momentum (through the Maxwell stress tensor), and conservation of angular momentum (through an angular momentum flux density tensor.) An interesting result in [61] is that the invariance of the momentum content per unit length arises from energy conservation, not conservation of momentum. This can be understood, however, given the fact that the energy flux density equals $c^{2}$ times the momentum density.

Lekner $[62,63]$ has considered the most general type of such beams and it is the properties of these which we shall briefly review. By taking a continuous superposition of the separable solutions of the Helmholtz equation we will obtain a general solution to the Helmholtz equation. This then gives a representation of a scalar beam as an integral of the separable solutions. The scalar beam solutions to the Helmholtz equation are [62]

$$
\psi_{m}(\mathbf{r})=e^{i m \phi} \int_{0}^{k} f(\kappa) e^{i q z} J_{m}(\kappa \rho) d \kappa, \quad q^{2}+\kappa^{2}=k^{2} .
$$


The invariants for the electromagnetic beams based on this wave function have been calculated in [62], with the constraint that only certain integrals of $|f(\kappa)|^{2}$ over $\kappa$ should exist. The beam solutions given in Eqn. 3.125 are known as generalized Bessel beams and it is the invariants of these beams which we shall discuss. We shall also derive a new invariant in the case of scalar generalized Bessel beams.

Conservation of energy of an electromagnetic beam is expressed in terms of the real fields $\mathbf{E}(\mathbf{r}, t), \mathbf{B}(\mathbf{r}, t)$ by

$$
\begin{aligned}
\nabla \cdot \mathbf{S} & +\frac{\partial u}{\partial t}=0, \\
\mathbf{S} & =\frac{c}{4 \pi}(\mathbf{E} \times \mathbf{B})=c^{2} \mathbf{p}, \\
u & =\frac{1}{8 \pi}\left(E^{2}+B^{2}\right) .
\end{aligned}
$$

(In these expressions Gaussian units for the fields have been used, see section 3.7 for the conversion to SI units.) The vector $\mathbf{S}$ is the well-known Poynting vector and represents the energy per unit time per unit area transported by the fields, $\mathbf{p}$ is the momentum density, and $u$ is the energy density of the electromagnetic fields which constitute the light beam.

It follows from Eqns. 3.126 that

$$
P_{z}^{\prime}=\int \bar{p}_{z} d^{2} r \quad \text { is an invariant, i.e. not dependent on } z \text {. }
$$

The bar is used to denote a cycle average, used here and throughout this section, and the $\int d^{2} r$ notation denotes $\int_{-\infty}^{\infty} \int_{-\infty}^{\infty} d x d y$ : integration over a transverse section of the beam, at fixed $z$. The proof may be found in [61]; it follows by applying $\int d^{2} r$ to the cycle average of the first equation in Eqns. 3.126 and using the definition of $\mathbf{S}$ in the second equation of Eqns. 3.126. In this interpretation $d P_{z}=P_{z}^{\prime} d z$ is the momentum content in a slice of thickness $d z$, thus the quantity $P_{z}^{\prime}$ is a natural measure of the momentum content per unit length along the $z$ direction. As stated earlier, the invariance of the momentum content per unit length is derived from energy conservation. 
The $z$ component of momentum content per unit length, $z$ component of angular momentum content per unit length, and energy content per unit length are defined as follows:

$$
\left\{\begin{aligned}
P_{z}^{\prime} & =\int \bar{p}_{z} d^{2} r \\
J_{z}^{\prime} & =\int(\mathbf{r} \times \overline{\mathbf{p}})_{z} d^{2} r \\
U^{\prime} & =\int \bar{u} d^{2} r
\end{aligned}\right.
$$

The energy and angular momentum content per unit length are not invariants in general: see, for example, [61] where an example is given. For the Barnett and Allen beam, however, these quantities are invariants.

TM, TE, and circularly polarized (CP) electromagnetic beams are studied in [62], based on the scalar solution given in Eqn. 3.125. The calculation of the electric and magnetic field for the dual TM and TE beams based on the scalar solutions of the Helmholtz equation has been given in section 3.7, further details may be found in [62]. The derivation of $P_{z}^{\prime}, J_{z}^{\prime}$, and $U^{\prime}$ in the case of a TM, or TE beam, requires the use of the various discontinuous integrals of Weber and Schafheitlin, which may be found in Chapter 11 of [30], or Chapter 13 of [64].

A summary of the results of [62] shall be presented here. Lekner [62] found that the quantities in Eqns. 3.128 are all invariants and are given by the following expressions

$$
\begin{gathered}
U^{\prime}=\frac{1}{4} \int_{0}^{k} \kappa|f(\kappa)|^{2} d \kappa . \\
c P_{z}^{\prime}=\frac{1}{4 k} \int_{0}^{k} \kappa q|f(\kappa)|^{2} d \kappa . \\
J_{z}^{\prime}=\frac{m}{4 c k} \int_{0}^{k} \kappa|f(\kappa)|^{2} d \kappa .
\end{gathered}
$$

An interesting feature of these expressions is that $U^{\prime} \geq c P_{z}^{\prime}$, since $q \leq k$. This is a general inequality proved in [61]. Another feature of these generalized Bessel beams is that for all TM and TE beams the energy content per unit length is independent of $m$. Comparison of Eqn. 3.129 and Eqn. 3.131 shows that $\omega J_{z}^{\prime}=m U^{\prime}$.

This relation between the angular momentum and energy is consistent with 
the idea of a beam consisting of photons of energy $\hbar \omega$ and angular momentum $m \hbar$.

Lekner [62] considered electromagnetic beams. In the case of scalar beams there is another invariant, namely the normalization integral. The normalization integral is not in general an invariant: see [16] for an example. However, the normalization integral for the scalar solutions given in Eqn. 3.125 is an invariant. The normalization integral in a cross-section of the beam is given by

$$
N=\int_{0}^{\infty} \rho|\psi|^{2} d \rho
$$

The following singular integral is needed to evaluate Eqn. 3.132 [64]

$$
\int_{0}^{\infty} \rho J_{m}(\kappa \rho) J_{m}\left(\kappa^{\prime} \rho\right) d \rho=\kappa^{-1} \delta\left(\kappa-\kappa^{\prime}\right) .
$$

On application of Eqn. 3.133 to Eqn. 3.132 the normalization integral becomes

$$
\begin{aligned}
N & =\int_{0}^{\infty} \rho d \rho \int_{0}^{k} f(\kappa) e^{i q z} J_{m}(\kappa \rho) d \kappa \int_{0}^{k} f^{*}\left(\kappa^{\prime}\right) e^{-i q^{\prime} z} J_{m}\left(\kappa^{\prime} \rho\right) d \kappa^{\prime} \\
& =\int_{0}^{k} \kappa^{-1}|f(\kappa)|^{2} d \kappa .
\end{aligned}
$$

Thus we have this new result that the scalar generalized Bessel solutions have another invariant associated with them. That is, the normalization integral of such beams is independent of the longitudinal coordinate $z$.

The simple product solutions given in Eqns. 3.123 correspond to a deltafunction amplitude in the wave function of Eqn. 3.125, which leads to divergent integrals for the physical quantities of the beam, such as the energy content per unit length. The simple product solutions of the Helmholtz equation are not physical beams in their own right, but certain superpositions of them, in the form of a generalized Bessel beam, can give finite physical properties. In the case of TM and TE beams the normal photon view of an electromagnetic beam is attained. This is, however, only realized in the TM or TE cases, the energy and angular momentum relationship is different in the case of a CP beam. 
This section has presented the generalized Bessel beams and summarized the results of [62] which derives the invariants for these electromagnetic beams. We also showed that the normalization integral is an invariant in the scalar beam case. The beams are interesting from several points of view. They satisfy the requirement of being purely forward propagating, a requirement which none of the spherical Bessel beams satisfy, and all of the oblate spheroidal beams satisfy. The generalized Bessel beams are highly symmetrical in that they have invariant momentum content per unit length, angular momentum content per unit length, and energy content per unit length along the $z$ direction of the beam.

The property of invariant energy and angular momentum content per unit length is not a general property of electromagnetic beams, but one which the generalized Bessel beams have. The downside of the generalized Bessel beams is that no weight function $f(\kappa)$ has been found which gives a simple analytical expression for the resulting scalar and electromagnetic beams. We showed in the previous section that the oblate spheroidal wave functions are not physical scalar or (TE and TM) electromagnetic beams. It may be that physical beams are in a form similar to the generalized Bessel beams, which would warrant investigation into determining a suitable weight function $f(\kappa)$.

\subsubsection{Bateman solution of the wave equation}

Exact solutions to the Helmholtz equation have been obtained as a product of angular and radial oblate spheroidal functions. Through a sum of the separable products all other solutions can, in principle, be obtained. However, there is a general solution to the Helmholtz equation which is not in the form of a product solution. This is the Bateman solution of the wave equation. The Bateman [65] solution gives an integral representation of the solution to the wave equation, which may be modified to represent a scalar beam solution of the Helmholtz equation. In what follows, we shall present the Bateman solution of the wave equation, and modify it to give a scalar beam solution. 
The Bateman solution to the wave equation, in cylindrical polar coordinates, is (p 402 [49]):

$$
\begin{aligned}
\psi(\rho, z, t) & =\int_{-\pi}^{\pi} f(z+i \rho \cos (\theta), c t+\rho \sin (\theta)) d \theta \\
& +\int_{0}^{b} \int_{-\pi}^{\pi} \operatorname{arcsinh}\left(\frac{a+z+c t \cos (\theta)}{\rho \sin (\theta)}\right) F(a, \theta) d \theta d a .(3 .
\end{aligned}
$$

The last term was included by Bateman to allow for logarithmic divergence in the solution when $\rho=0$. The last term also satisfies the wave equation without the integration, but the first term needs the integration over $\theta$, since $\left(\nabla^{2}-1 / c^{2} \partial_{t}^{2}\right) f(z+i \rho \cos (\theta), c t+\rho \sin (\theta))=-1 / \rho^{2} \partial_{\theta}^{2} f(z+i \rho \cos (\theta), c t+$ $\rho \sin (\theta)$ ), which upon integration gives zero.

For a monochromatic beam the time dependence is $\exp (-i \omega t)=\exp (-i c k t)$, which restricts the function $f$ to be of the form

$$
f=\frac{1}{2 \pi} g(z+i \rho \cos (\theta)) e^{-i k(c t+\rho \sin (\theta))} .
$$

Therefore $\left(\nabla^{2}+k^{2}\right) \psi=0$ is solved by

$$
\psi(\rho, z)=\frac{1}{2 \pi} \int_{-\pi}^{\pi} g(z+i \rho \cos (\theta)) e^{-i k \rho \sin (\theta)} d \theta .
$$

In the case of scalar beams the second term of Eqn. 3.135 is not needed, since we do not require the possibility of divergence in $\psi$ when $\rho=0$. Eqn. 3.137 is the most general (non-singular) form of the solution to the Helmholtz equation, but it does not give an insight into what function $g(z+i \rho \cos (\theta))$ gives a physical beam solution.

In section 3.5 the solution to the radial equation was expressed as an integral of an angular function. The kernel in the integral relation satisfies the Helmholtz equation Eqn. 3.59. Therefore, the Bateman form of the solution to the Helmholtz equation given in Eqn. 3.137 can be used to derive the kernels of the integral relations. The simplest kernel is the plane wave solution $\exp (i k z)$, which was used in expressing the integral relations of Eqn. 3.60 and Eqn. 3.61 between the radial and angular confluent Heun functions. We shall now derive the plane wave solution utilizing the Bateman solution Eqn. 3.137. 
If $g=e^{i k(z+i \rho \cos (\theta))}$ the solution to the Helmholtz equation becomes

$$
\psi(\rho, z)=\frac{\exp (i k z)}{2 \pi} \int_{-\pi}^{\pi} e^{-k \rho(\cos (\theta)+i \sin (\theta))} d \theta .
$$

Performing the integration is best handled using contour integration. If we make the substitution $X=\exp (i \theta), d X=i X d \theta$, and perform the contour integration around the unit circle, the solution is

$$
\psi(\rho, z)=\frac{\exp (i k z)}{2 \pi i} \oint \frac{\exp (-k \rho X)}{X} d X .
$$

The residue at the singularity $X=0$ is given by 1 . By the residue theorem, or Cauchy's integral formula, the solution to the Helmholtz equation, for this particular $g$, is

$$
\psi(\rho, z)=\exp (i k z)
$$

which is $\exp (i \beta \xi \eta)$ in oblate spheroidal coordinates. This gives the usual plane wave solution, which can be obtained by inspection of the partial differential equation. However, this method illustrates an application of the Bateman solution. In oblate spheroidal coordinates the Bateman solution Eqn. 3.137 becomes

$\psi(\xi, \eta)=\frac{1}{2 \pi} \int_{-\pi}^{\pi} g\left(b \xi \eta+i b \sqrt{\left(\xi^{2}+1\right)\left(1-\eta^{2}\right)} \cos (\theta)\right) e^{-i \beta \sin (\theta) \sqrt{\left(\xi^{2}+1\right)\left(1-\eta^{2}\right)}} d \theta$.

In conclusion, the Bateman solution in Eqn. 3.137 is a general solution of the Helmholtz equation. For a certain $g$, the Bateman solution may lead to an analytic expression for exact scalar beams. Thus, in future research, it may be of benefit to further investigate various functions $g$, and determine the properties of the corresponding scalar beams. 


\section{Chapter 4}

\section{Scattering of scalar waves by spheroids}

Chapter 3 investigated the applicability of oblate spheroidal wave functions as free space scalar beams. As discussed in the introduction, these wave functions have also been used for solving the scattering of acoustic waves by spheroidal obstacles. In this Chapter, however, we shall give a phase shift formulation of the scattering of Schrödinger particle waves by impenetrable spheroids, and for scattering of sound waves by acoustically soft spheroids. The important step is the application of a finite Legendre transform to the Helmholtz equation. We shall show that the resulting radial eigenfunctions, in the oblate and prolate cases, satisfy certain differencedifferential equations. In the long-wavelength limit solution of the differencedifferential equations in terms of Legendre functions is possible. This shall lead to expressions for the scattering length, differential scattering crosssection, and the total cross-section, of spheroidal scatterers, in the longwavelength limit. The method is restricted to scattering by spheroids whose symmetry axis is coincident with the direction of the incident plane wave. The advantage of the method is that it is in one-to-one correspondence with the partial wave theory of scattering by spherical objects developed by Rayleigh. 


\subsection{Introduction}

In classical mechanics the elastic scattering of particles by collisions is determined by the particle's velocity and impact parameter. The classical scattering problem is to determine the scattering angle in terms of the impact parameter. However, in quantum mechanics, the concept of a definite path is meaningless, and the very notion of an impact parameter is also not well defined. In quantum scattering theory the aim is to compute the probability that a certain particle, represented by its deBroglie wave, is scattered through a certain angle. This probability is obtained from the scattering amplitude, which is related to the experimentally important total cross-section in a simple way. A thorough treatment of quantum scattering by spherical scatterers is given in Chapter 17 of [29]. In this Chapter we shall summarize the usual phase shift theory of scattering by spherical scatterers, and then derive analogous results for the scattering by spheroidal obstacles.

We shall investigate the scattering of an incident Schrödinger particle wave, represented as a plane wave $e^{i k z}$, by hard oblate or prolate spheroids. In this context the spheroids are impenetrable and the extinction of the incident plane wave is due entirely to scattering, with no contribution from absorption. The results are not restricted to scattering of quantum particle waves, but are relevant to scattering of acoustic sound waves by acoustically soft spheroidal objects. This is because in both cases the boundary condition is that the wave function, or velocity potential in the acoustic case, must vanish on the surface of the spheroid.

Before deriving the theory for spheroidal scatterers, it is instructive to summarize the main results for the scattering of waves by spherical scatterers in terms of the phase shifts $\delta_{n}$. The principle behind the phase shift formulation of scattering is based on conservation of probability. A Schrödinger particle wave, scattered by a spherical obstacle, may be represented by a superposition of scattered partial waves, each with only a change in the phase of the incident particle wave. 
The Schrödinger equation is separable in spherical coordinates, and so the solution can be represented as a sum over products of a radial and angular function

$$
\psi(r, \theta)=\sum_{n=0}^{\infty}(2 n+1) R_{n}(r) P_{n}(\cos (\theta)) .
$$

The physical solutions to Schrödinger's equation must have the following asymptotic form

$$
\psi \rightarrow e^{i k z}+f(\theta) \frac{e^{i k r}}{r},
$$

where $f(\theta)$ is the scattering amplitude. This expression represents the wave function as a superposition of the incident wave plus the outward propagating scattered wave. The asymptotic form of the radial solutions are given in terms of the phase shifts as

$$
R_{n}(r) \rightarrow \frac{i^{n}}{k r} e^{i \delta_{n}} \sin \left(k r-n \pi / 2+\delta_{n}\right)
$$

The difference between $\psi$ and $e^{i k z}$ gives the scattered wave. In order to derive the scattering amplitude the incident plane wave must be represented in terms of an expansion of the solutions of Schrödinger's equation in spherical coordinate eigenstates. This is known as Rayleigh's formula:

$$
e^{i k z}=\sum_{n=0}^{\infty}(2 n+1) i^{n} j_{n}(k r) P_{n}(\cos (\theta)) .
$$

Thus the scattering amplitude can be found by using the asymptotic form of the radial solutions along with the Rayleigh formula. The scattering amplitude is

$$
f(\theta)=\frac{1}{k} \sum_{n=0}^{\infty}(2 n+1) e^{i \delta_{n}} \sin \left(\delta_{n}\right) P_{n}(\cos (\theta)) .
$$

Once the scattering amplitude has been determined we are able to deduce the differential scattering cross-section defined as follows

$$
D(\theta)=\frac{d \sigma}{d \Omega}=|f(\theta)|^{2} .
$$

If we integrate the differential scattering cross-section over the full solid angle we obtain the total cross-section. 
The total cross-section is the ratio of the total probability (per unit time) that a particle will be scattered to the probability current density in the incident wave. It is effectively the total area of incident beam scattered by the target. The total cross-section is defined as follows

$$
\sigma=\int D(\theta) d \Omega=2 \pi \int_{0}^{\pi}|f(\theta)|^{2} \sin (\theta) d \theta .
$$

In terms of the phase shifts $\delta_{n}$ the total cross-section is

$$
\sigma=\frac{4 \pi}{k^{2}} \sum_{n=0}^{\infty}(2 n+1) \sin ^{2}\left(\delta_{n}\right) .
$$

The quantum theory of scattering can be found in Chapter 17 of [29], or Chapter 11 of [57]. Chapter 4 of [40] gives the classical theory of scattering. Scattering by spherical obstacles can be summarized by the following set of equations:

$$
\begin{aligned}
\psi(r, \theta) & =\sum_{n=0}^{\infty}(2 n+1) R_{n}(r) P_{n}(\cos (\theta)), \\
\psi(r, \theta) & \rightarrow e^{i k z}+f(\theta) \frac{e^{i k r}}{r} \\
R_{n}(r) & \rightarrow \frac{i^{n}}{k r} e^{i \delta_{n}} \sin \left(k r-n \pi / 2+\delta_{n}\right), \\
f(\theta) & =\frac{1}{k} \sum_{n=0}^{\infty}(2 n+1) e^{i \delta_{n}} \sin \left(\delta_{n}\right) P_{n}(\cos (\theta)), \\
\frac{d \sigma}{d \Omega} & =|f(\theta)|^{2}, \\
\sigma & =\int \frac{d \sigma}{d \Omega} d \Omega=\frac{4 \pi}{k^{2}} \sum_{n=0}^{\infty}(2 n+1) \sin ^{2}\left(\delta_{n}\right) .
\end{aligned}
$$

Scattering theory reduces to calculating the phase shifts $\delta_{n}$ for a specific potential. This is done by solving the Schrödinger equation in the region where the potential is non-zero, and then using appropriate boundary conditions. 
These equations (in order) express the solution of Helmholtz's equation $\left(\nabla^{2}+k^{2}\right) \psi=0$ as a sum over products of radial and angular solutions, give the asymptotic form appropriate for an incoming plane wave and a spherically diverging wave, express the asymptotic form of the radial solutions in terms of the phase shifts $\delta_{n}$, give the scattering amplitude $f(\theta)$ in terms of the phase shifts, and give the differential and total cross-sections.

It follows from comparison of Eqn. 4.5 and Eqn. 4.8 that the forward scattering amplitude is related to the total cross-section:

$$
\sigma=\frac{4 \pi}{k} \operatorname{Im}\{f(0)\} .
$$

This result, called the 'optical theorem', holds for elastic scattering (no absorption) of scalar waves, for which the phase shifts $\delta_{n}$ are real.

In the case of scattering by spheroids, which is our main focus, we seek to derive expressions analogous to those given in Eqns. 4.9 for scattering by spherical scatterers.

\subsection{Wave equation for oblate spheroidal scat- terers}

The oblate spheroidal coordinate system has been explained in Chapter 3, which should be referred to for details about the coordinate system. In this Chapter, however, we shall use a different range for the variables. That is

$$
\begin{array}{r}
\rho=b \sqrt{\xi^{2}+1} \sqrt{1-\eta^{2}}, \quad z=b \xi \eta, \quad \phi=\phi, \\
-1 \leq \eta \leq 1, \quad 0 \leq \xi<\infty, \quad 0 \leq \phi \leq 2 \pi .
\end{array}
$$

The definitions of the oblate spheroidal coordinate system given in Eqns. 3.14 and Eqns. 4.11 are both representations of the whole of space. However, Eqns. 3.14 have a branch cut on the $\eta=0$ axis, whereas Eqns. 4.11 have a branch cut on the disk $\xi=0$. 

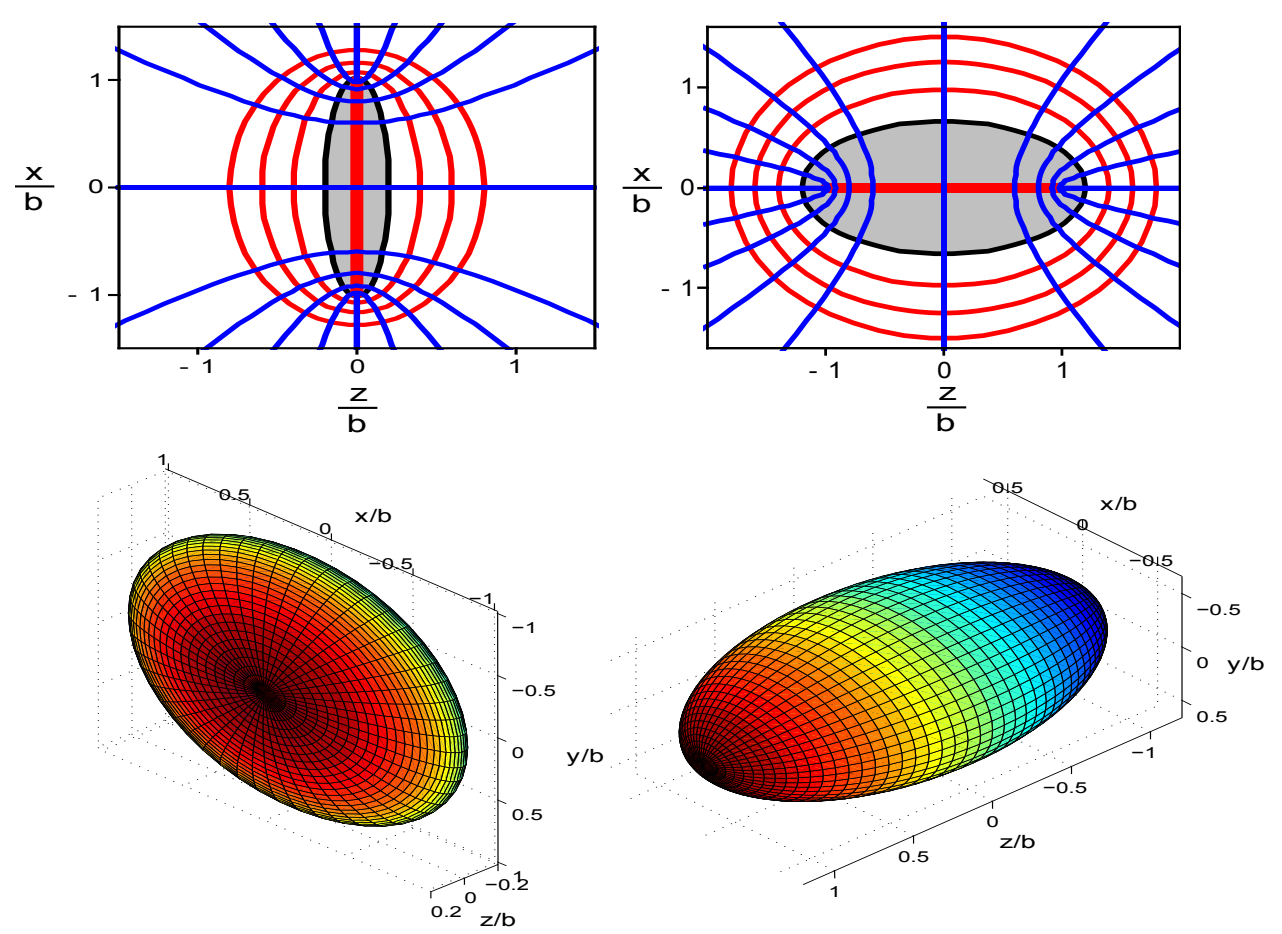

Figure 4.1: Oblate and prolate spheroidal coordinate systems (upper left and right, respectively), and two examples of these spheroids. Lower left: the oblate spheroid $\xi_{0}=0.2$. Lower right: the prolate spheroid $\xi_{0}=1.2$. In the upper figures these solid bodies are the innermost spheroids, shown shaded. The parameter $\xi_{0}$ increases in steps of 0.2 from the inner spheroids.

The form of the oblate spheroidal coordinate system in Eqns. 3.14 is used for beam solutions, propagating along the $z$ axis, whereas the form in Eqns. 4.11 is used for outward propagating waves, which are observed in the scattering problems under consideration. The ellipsoids represented by the oblate spheroidal coordinate system, Eqn. 3.15, are shown in Fig. 4.1.

As discussed in Chapter 3, the free space Schrödinger equation is equivalent to the scalar Helmholtz equation. We shall consider plane waves incident along the symmetry axis of the spheroid, so this restricted problem has no $\phi$-dependence. The corresponding Helmholtz equation in oblate spheroidal coordinates is

$$
\left\{\partial_{\xi}\left(1+\xi^{2}\right) \partial_{\xi}+\partial_{\eta}\left(1-\eta^{2}\right) \partial_{\eta}+\beta^{2}\left(\xi^{2}+\eta^{2}\right)\right\} \psi(\xi, \eta)=0
$$


The product of the wavenumber $k$ and parameter $b$ is, as in Chapter 3, denoted by $\beta$. The Helmholtz partial differential equation, Eqn. 4.12, is separable, which is the usual method of proceeding. Instead, we perform a finite Legendre transformation of Eqn. 4.12, see, for example, section 6.11 of [66]. The finite Legendre transform of $\psi(\xi, \eta)$ is defined by

$$
R_{n}(\xi)=\frac{1}{2} \int_{-1}^{1} \psi(\xi, \eta) P_{n}(\eta) d \eta
$$

where $P_{n}(\eta)$ is the Legendre polynomial of order $n$, and satisfies

$$
\left[\partial_{\eta}\left(1-\eta^{2}\right) \partial_{\eta}+n(n+1)\right] P_{n}(\eta)=0 .
$$

It follows from integration by parts and the use of Eqn. 4.13 and Eqn. 4.14 that

$$
\frac{1}{2} \int_{-1}^{1}\left[\partial_{\eta}\left(1-\eta^{2}\right) \partial_{\eta} \psi(\xi, \eta)\right] P_{n}(\eta) d \eta=-n(n+1) R_{n}(\xi)
$$

The Legendre polynomials satisfy the following recurrence relation, also given in Eqn. 2.25,

$$
\begin{aligned}
(2 n+1) \eta^{2} P_{n} & =\frac{n(n-1)}{2 n-1} P_{n-2}+\left\{\frac{(n+1)^{2}}{2 n+3}+\frac{n^{2}}{2 n-1}\right\} P_{n} \\
& +\frac{(n+1)(n+2)}{2 n+3} P_{n+2} .
\end{aligned}
$$

Therefore operating on the Helmholtz equation with $\frac{1}{2} \int_{-1}^{1} P_{n}(\eta) d \eta$, and using the definition of $R_{n}(\xi)$, we obtain the following coupled set of differencedifferential equations for $R_{n}(\xi)$

$$
\begin{aligned}
& {\left[\partial_{\xi}\left(1+\xi^{2}\right) \partial_{\xi}+\beta^{2} \xi^{2}-n(n+1)\right] R_{n}(\xi) } \\
+ & \frac{\beta^{2}}{2 n+1}\left\{\frac{n(n-1)}{2 n-1} R_{n-2}(\xi)+\left[\frac{(n+1)^{2}}{2 n+3}+\frac{n^{2}}{2 n-1}\right] R_{n}(\xi)\right. \\
+ & \left.\frac{(n+1)(n+2)}{2 n+3} R_{n+2}(\xi)\right\}=0 .
\end{aligned}
$$

The first three of the set Eqn. 4.17 read

$$
\begin{aligned}
& n=0:\left[\partial_{\xi}\left(1+\xi^{2}\right) \partial_{\xi}+\beta^{2}\left(\xi^{2}+\frac{1}{3}\right)\right] R_{0}+\frac{2}{3} \beta^{2} R_{2}=0 . \\
& n=1:\left[\partial_{\xi}\left(1+\xi^{2}\right) \partial_{\xi}+\beta^{2}\left(\xi^{2}+\frac{3}{5}\right)-2\right] R_{1}+\frac{2}{5} \beta^{2} R_{3}=0 . \\
& n=2:\left[\partial_{\xi}\left(1+\xi^{2}\right) \partial_{\xi}+\beta^{2}\left(\xi^{2}+\frac{11}{21}\right)-6\right] R_{2}+\frac{\beta^{2}}{5}\left(\frac{12}{7} R_{4}+\frac{2}{3} R_{0}\right)=0 .
\end{aligned}
$$


We do not have the general solution of this system of equations, but the long-wavelength limit $(\beta=k b \rightarrow 0)$ is simple:

$$
\left(1+\xi^{2}\right) R_{n}^{\prime \prime}+2 \xi R_{n}^{\prime}-n(n+1) R_{n}=0 .
$$

Comparison of Eqn. 4.19 with the Legendre differential equation Eqn. 4.14 shows that, at low frequencies, $R_{n}$ becomes a linear combination of the regular and singular Legendre functions of imaginary arguments:

$$
R_{n}(\xi) \rightarrow p_{n} P_{n}(i \xi)+q_{n} Q_{n}(i \xi), \quad \beta \rightarrow 0 .
$$

Since

$$
Q_{0}(x)=\frac{1}{2} \ln \frac{1+x}{1-x} \quad \text { and } \quad \frac{1}{2 i} \ln \frac{1+i \xi}{1-i \xi}=\arctan (\xi)
$$

we can write the first three low-frequency limiting forms of $R_{n}$ as

$$
\begin{aligned}
& R_{0} \rightarrow p_{0}+q_{0} \arctan (\xi), \\
& R_{1} \rightarrow p_{1} \xi+q_{1}(1+\xi \arctan (\xi)), \\
& R_{2} \rightarrow p_{2}\left(1+3 \xi^{2}\right)+q_{2}\left[3 \xi+\left(1+3 \xi^{2}\right) \arctan (\xi)\right] .
\end{aligned}
$$

In fact, $R_{n}(\xi)=P_{n}(i \xi)$ solves Eqn. 4.17 for all $n \geq 0$, and $R_{n}(\xi)=Q_{n}(i \xi)$ solves Eqn. 4.17 for all $n \geq 2$ (the recurrence relation Eqn. 4.16 is satisfied by $Q_{n}(i \xi)$ only for $n \geq 2$ ), but these are not physical solutions except in the long-wavelength limit.

At high frequencies the behaviour of $R_{n}(\xi)$ is more like that of the spherical Bessel functions $j_{n}(\beta \xi)$ and $y_{n}(\beta \xi)$, which satisfy the equation

$$
\xi^{2} \frac{d^{2} j_{n}}{d \xi^{2}}+2 \xi \frac{d j_{n}}{d \xi}+\left[\beta^{2} \xi^{2}-n(n+1)\right] j_{n}=0 .
$$

The remainders when $R_{n}(\xi)$ is set equal to $j_{n}(\beta \xi)$ in Eqn. 4.17 are

$$
\begin{array}{ll}
n=0: & \frac{4}{3} \beta^{2} j_{2}(\beta \xi), \\
n=1: & \frac{4}{5} \beta^{2} j_{3}(\beta \xi), \\
n=2: & \frac{4}{5} \beta^{2}\left[\frac{6}{7} j_{4}(\beta \xi)+\frac{1}{3} j_{0}(\beta \xi)\right] .
\end{array}
$$

Corresponding results hold for $R_{n}(\xi)$ set equal to $y_{n}(\beta \xi)$. 


\subsection{Scattering by oblate spheroids}

In this section we shall formulate the scattering of waves incident along the symmetry axis of an oblate spheroid in a similar form as the phase shift formulation of scattering by spherical scatterers [29]. The theory of scattering by spherically symmetric potentials reduces to calculating the phase shifts, as functions of the wavelength, for a specific potential.

The inverse of the Legendre transform Eqn. 4.13 (into which the factor $\frac{1}{2}$ has been inserted to retain exact correspondence with scattering theory) is obtained from the orthogonality relation

$$
\int_{-1}^{1} P_{n}(\eta) P_{m}(\eta) d \eta=\frac{2 \delta_{n m}}{2 n+1},
$$

where $\delta_{n m}$ is the Kronecker delta function. By analogy with Eqn. 4.1 we write

$$
\psi(\xi, \eta)=\sum_{n=0}^{\infty}(2 n+1) R_{n}(\xi) P_{n}(\eta) .
$$

If we multiply both the left and right hand sides of Eqn. 4.26 by the Legendre polynomial $P_{m}(\eta)$ and invoke the orthogonality condition of Legendre polynomials this gives us back Eqn. 4.13.

In analogy with the spherical scattering theory we replace $k r$ by $\beta \xi=$ $(k b \xi)$ and $\cos (\theta)$ by $\eta$; thus we require the wave function to have the following asymptotic form

$$
\psi(\xi, \eta) \rightarrow e^{i \beta \xi \eta}+F(\eta) \frac{e^{i \beta \xi}}{\beta \xi},
$$

where $F(\eta)$ is the (dimensionless) spheroidal scattering amplitude. The scattering amplitude gives the probability of scattering in a given direction specified asymptotically by $\eta$.

As in Eqn. 4.3, the asymptotic form of the radial functions $R_{n}(\xi)$, in terms of the phase shifts $\delta_{n}$, is

$$
R_{n}(\xi) \rightarrow \frac{i^{n}}{\beta \xi} e^{i \delta_{n}} \sin \left(\beta \xi-n \pi / 2+\delta_{n}\right) .
$$


In order to derive the scattering amplitude $F(\eta)$, in terms of the phase shifts, we need the expansion of the plane wave $e^{i k z}$ in terms of the Legendre polynomials, and an undetermined radial function. Expanding the incident plane wave as in Eqn. 4.26 we have

$$
e^{i \beta \xi \eta}=\sum_{n=0}^{\infty}(2 n+1) G_{n}(\xi) P_{n}(\eta) .
$$

The function $G_{n}(\xi)$ can be found as before by multiplying both the left and right sides of Eqn. 4.29 by the Legendre polynomial $P_{m}(\eta)$, integrating, and invoking the orthogonality of the Legendre polynomials. This gives

$$
G_{n}(\xi)=\frac{1}{2} \int_{-1}^{1} e^{i \beta \xi \eta} P_{n}(\eta) d \eta=\frac{1}{(-i)^{n}} j_{n}(\beta \xi),
$$

from the integral representation of the spherical Bessel function $j_{n}(\beta \xi)$. We now have the desired expansion of the plane wave $\left(e^{i k z}\right)$, in terms of spherical Bessel functions and Legendre polynomials,

$$
e^{i \beta \xi \eta}=\sum_{n=0}^{\infty}(2 n+1) i^{n} j_{n}(\beta \xi) P_{n}(\eta) .
$$

Eqn. 4.31 is in exact analogy with the familiar Rayleigh formula Eqn. 4.4, which expands a plane wave in terms of the same functions, except written in spherical coordinates. Now that the plane wave expansion expressed in spheroidal coordinates has been found we may determine the scattering amplitude from the asymptotics of the wave function.

The asymptotics of the spherical Bessel functions are

$$
j_{n}(\beta \xi) \rightarrow \frac{\sin \left(\beta \xi-\frac{n \pi}{2}\right)}{\beta \xi}, \quad \beta \xi \rightarrow \infty .
$$

Therefore the plane wave expansion in the limit that $\beta \xi \rightarrow \infty$ is

$$
e^{i \beta \xi \eta} \rightarrow \sum_{n=0}^{\infty}(2 n+1) i^{n} \frac{\sin \left(\beta \xi-\frac{n \pi}{2}\right)}{\beta \xi} P_{n}(\eta) .
$$

The scattering amplitude $F(\eta)$ can be found from the difference in the asymptotic expression of $\psi(\xi, \eta)-e^{i \beta \xi \eta}$. This gives us: 


$$
\begin{aligned}
\psi(\xi, \eta)-e^{i \beta \xi \eta} & =\sum_{n=0}^{\infty}(2 n+1)\left[R_{n}(\xi)-i^{n} j_{n}(\beta \xi)\right] P_{n}(\eta), \\
& \rightarrow \frac{1}{\beta \xi} \sum_{n=0}^{\infty} i^{n}(2 n+1)\left[e^{i \delta_{n}} \sin \left(\beta \xi-n \pi / 2+\delta_{n}\right)\right. \\
& -\sin (\beta \xi-n \pi / 2)] P_{n}(\eta), \\
& =\frac{e^{i \beta \xi}}{\beta \xi} \sum_{n=0}^{\infty}(2 n+1) e^{i \delta_{n}} \sin \left(\delta_{n}\right) P_{n}(\eta) .
\end{aligned}
$$

As the scattering amplitude is the coefficient of the scattered wave, inspection of Eqn. 4.34 gives

$$
F(\eta)=\sum_{n=0}^{\infty}(2 n+1) e^{i \delta_{n}} \sin \left(\delta_{n}\right) P_{n}(\eta) .
$$

Now that the scattering amplitude has been found we can deduce the differential scattering cross-section using Eqn. 4.6 and Eqn. 4.35. The result is

$$
\frac{d \sigma}{d \Omega}=\frac{1}{k^{2}}\left|\sum_{n=0}^{\infty}(2 n+1) e^{i \delta_{n}} \sin \left(\delta_{n}\right) P_{n}(\eta)\right|^{2} .
$$

Integration of the differential scattering cross-section gives the total crosssection

$$
\begin{aligned}
\sigma & =2 \pi \int_{-1}^{1} \frac{d \sigma}{d \Omega} d \eta \\
& =\frac{4 \pi}{k^{2}} \sum_{n=0}^{\infty}(2 n+1) \sin ^{2}\left(\delta_{n}\right)
\end{aligned}
$$

We may now derive the optical theorem, which relates the total cross-section to the forward scattering amplitude, for the case of scattering by spheroids. Using Eqn. 4.35 and Eqn. 4.37, the total cross-section is given by

$$
\sigma=\frac{4 \pi}{k^{2}} \operatorname{Im}\{F(\eta=1)\} .
$$

The scattering theory of spheroids is completely analogous to the scattering by spherically symmetric potentials. The analogy between scattering amplitudes is given by the following relation

$$
\frac{F(\eta)}{\beta \xi} \leftrightarrow \frac{f(\theta)}{r}
$$


This formalism has now expressed the free space solutions to Helmholtz's equation in terms of a product of a Legendre polynomial and an unknown function $R_{n}(\xi)$. If we can determine $R_{n}(\xi)$ then by Eqn. 4.28 we can obtain the phase shifts $\delta_{n}$ from the asymptotic form of $R_{n}(\xi)$. This would enable the scattering amplitude to be subsequently deduced.

The next sections investigate the case of long-wavelength scattering by oblate and prolate spheroids. In this limit the exact solutions for the radial eigenfunctions, namely the Legendre functions, have the correct behaviour. For long-wavelength scattering only $\delta_{0}$ is important. The theory has been derived here in its complete form, however, using all the phase shifts and the full scattering amplitude. The scattering by spheroidal obstacles may be summarized in terms of the phase shifts as follows:

$$
\begin{aligned}
\psi(\xi, \eta) & =\sum_{n=0}^{\infty}(2 n+1) R_{n}(\xi) P_{n}(\eta), \\
\psi(\xi, \eta) & \rightarrow e^{i \beta \xi \eta}+F(\eta) \frac{e^{i \beta \xi}}{\beta \xi} \\
R_{n}(\xi) & \rightarrow \frac{i^{n}}{\beta \xi} e^{i \delta_{n}} \sin \left(\beta \xi-n \pi / 2+\delta_{n}\right), \\
F(\eta) & =\sum_{n=0}^{\infty}(2 n+1) e^{i \delta_{n}} \sin \left(\delta_{n}\right) P_{n}(\eta), \\
\frac{d \sigma}{d \Omega} & =k^{-2}|F(\eta)|^{2}, \\
\sigma & =\int \frac{d \sigma}{d \Omega} d \Omega=\frac{4 \pi}{k^{2}} \sum_{n=0}^{\infty}(2 n+1) \sin ^{2} \delta_{n} .
\end{aligned}
$$

\subsection{Long wave scattering by oblate spheroids}

If we consider the case of a wave incident on an oblate spheroid, in the longwavelength limit, then it is only the lowest partial wave amplitude which is significant. This is called $s$ wave scattering and arises as a result of the partial wave amplitudes being directly proportional to the wavenumber $k, \mathrm{p} 546$ of [29]. If we adapt this to the spheroidal case under consideration, then the solution $R_{0}(\xi)$ shall dominate, and we may neglect the other $R_{n}(\xi)(n \neq 0)$. 
In the long-wavelength limit, for spherical scatterers, the scattering amplitude $f(\theta)$ is approximately given by the lowest partial wave amplitude $f_{0}$. This is usually termed the scattering length $s$ and is defined as follows

$$
s=-\lim _{k \rightarrow 0} \frac{\delta_{0}}{k} .
$$

Physically the scattering length equals minus the amplitude of the scattered wave in the long-wavelength limit. The scattering length is independent of angle, which implies that in the long-wavelength limit the scattering is isotropic and independent of the incident particle's energy.

In the case of scattering by spherical scatterers the asymptotic form of the dominant $n=0$ wave, outside the scatterer, is

$$
R_{0} \rightarrow \frac{e^{i \delta_{0}}}{k r} \sin \left(k r+\delta_{0}\right)=\frac{e^{-i k s}}{k r} \sin k(r-s) .
$$

If we multiply Eqn. 4.42 by $r$ then, as $\lambda=2 \pi / k \rightarrow \infty$,

$$
r R_{0} \rightarrow r-s \quad \text { (outside the scatterer, but with } k(r-s)<<1 \text { ). }
$$

We shall adapt this to the case of scattering by spheroids. From Eqn. 4.28 the dominant partial wave, in the long-wavelength limit, has the asymptotic form

$$
R_{0} \rightarrow \frac{e^{i \delta_{0}}}{\beta \xi} \sin \left(\beta \xi+\delta_{0}\right)=\frac{e^{-i k s}}{\beta \xi} \sin \beta(\xi-s / b) .
$$

Thus the analogue of Eqn. 4.43 is

$$
\xi R_{0}(\xi) \rightarrow \xi-\xi_{s}, \quad \xi_{s}=s / b \quad\left[\beta\left(\xi-\xi_{s}\right)<<1\right] .
$$

Now that we have a definition of the scattering length for the scattering by oblate spheroidal objects we can obtain the total cross-section, in the long-wavelength limit.

For the acoustically soft, or quantum-mechanically hard, scattering by an oblate spheroid, the boundary condition on the spheroid $\xi=\xi_{0}$ is $\psi\left(\xi_{0}, \eta\right)$ $=0$. In the long-wavelength limit this implies $R_{0}\left(\xi_{0}\right)=0$. In order to have a solution which vanishes on the spheroid $\xi=\xi_{0}$ we take $R_{0}(\xi)$ (up to a constant factor), given in Eqns. 4.22, as

$$
R_{0}(\xi)=\arctan (\xi)-\arctan \left(\xi_{0}\right)
$$


This solution is correct in the long-wavelength limit, however, it clearly does not have the asymptotic form of the general solution derived in Eqn. 4.28. The solution does satisfy the boundary condition, $R_{0}\left(\xi_{0}\right)=0$, which is all that we require in order to derive the scattering length. The Taylor series expansion of Eqn. 4.46 for large $\xi$ (but $\beta \xi$ still small) is

$$
\begin{aligned}
\xi R_{0}(\xi) & =\xi\left(\frac{\pi}{2}-\arctan \frac{1}{\xi}-\arctan \xi_{0}\right), \\
& =\xi\left(\frac{\pi}{2}-\arctan \xi_{0}\right)-1+O\left(\xi^{-2}\right), \\
& =\xi\left(\arctan \frac{1}{\xi_{0}}\right)-1+O\left(\xi^{-2}\right) .
\end{aligned}
$$

Thus $\xi_{s}=\left(\arctan \xi_{0}^{-1}\right)^{-1}$, and the scattering length is

$$
s=b \xi_{s}=\frac{b}{\arctan \left(1 / \xi_{0}\right)} .
$$

Now that the scattering length has been deduced we may derive the differential scattering cross-section. In the oblate spheroid case we find

$$
\frac{d \sigma}{d \Omega}=b^{2} \xi_{s}^{2}=\frac{b^{2}}{\left(\arctan \left(1 / \xi_{0}\right)\right)^{2}} .
$$

The impenetrable disk $(\rho \leq b, z=0)$ is the limiting case of an oblate spheroid with $\xi_{0} \rightarrow 0$, and so comparison with Rayleigh's result [19] can be made. Setting $\xi_{0}=0$ in Eqn. 4.48 we have

$$
s=\frac{2 b}{\pi}, \quad \frac{d \sigma}{d \Omega}=\left(\frac{2 b}{\pi}\right)^{2},
$$

in agreement with [19].

Although this result has been known since 1897, the method used by Rayleigh is different from that used here. Rayleigh [19, 67] used a scalar theory of diffraction to solve the long-wavelength diffraction of an acoustic wave through an aperture. The boundary condition on the screen is acoustically hard, which corresponds to the normal derivative vanishing on the screen. The diffracted wave is then expressed as a certain surface integral over the aperture. 
By considering points situated on the aperture, along with the continuity of the velocity potential across the aperture, the surface integral is found to be equivalent to a distribution of charge density at unit potential. The amplitude of the diffracted wave is then readily deduced as an electrical capacitance, and from the known capacity of a conducting disk having the size and shape as the aperture, the diffracted wave can be inferred. The problem of soft acoustic scattering by a disk has an affinity to this, by using his aperture solution (with the superposition of the incident wave), Rayleigh is able to solve the acoustically soft scattering by a disk.

The differential scattering cross-section for an oblate spheroid, in the long-wavelength limit, has been found by Senior [22]. Senior expands the scattered wave in powers of the wavenumber $k$. By considering points near the boundary of the spheroid, Senior obtains a constraint based on a surface integral over the spheroid. The surface integral is identical to the equation for the surface charge distribution on a metallic conductor at unit potential and thus is related to the capacitance of the object of the same geometry. Senior derives this capacitance and obtains results equivalent to those found in Eqn. 4.49. The derivation given here, however, parallels the usual quantum scattering theory for spherically symmetric potentials.

The other limit is the case of large $b \xi_{0}$, which corresponds to the scattering by a sphere of radius $a=b \xi_{0}$. In the case of large $\xi_{0}$, i.e. $\xi_{0}>>1$, $\arctan \left(1 / \xi_{0}\right) \rightarrow 1 / \xi_{0}$. This gives the scattering length as $s=b \xi_{0}$ and thus the differential scattering cross-section, and the total cross-section, are

$$
\frac{d \sigma}{d \Omega} \rightarrow a^{2}, \quad \sigma \rightarrow 4 \pi a^{2},
$$

which is in agreement with the known total cross-section for hard sphere scattering $[29,57]$.

For the case of long-wavelength scattering by (quantum-mechanically) hard spheres, the total cross-section tends to the surface area of the sphere, and not the geometric cross-section of the sphere, with higher order terms proportional to powers of $k a$, where $a$ is the radius of the sphere. This result can be interpreted as the incident wave 'wrapping around' the sphere, p 404 [57]. 
It is actually true, in general, in the case of scattering by spherical scatterers, that the number of particles scattered is four times the number of particles in the incident particle flux, which is a purely quantum effect due to interference between the incident and scattered waves [29]. On the basis that long waves 'wrap around' the spheroid, we might expect the total cross-section to be proportional to the surface area of the oblate spheroid.

The spheroid $\xi=\xi_{0}$, given in Eqn. 3.15, has semi axes $a, a, c$ where $a=b\left(\xi_{0}^{2}+1\right)^{1 / 2}, c=b \xi_{0}$. The surface area of this oblate spheroid is

$$
A=2 \pi b^{2}\left\{\xi_{0}^{2}+1+\frac{\xi_{0}^{2}}{2 e} \ln \frac{1+e}{1-e}\right\}
$$

where $e$ is the ellipticity defined as

$$
e=\left(1-\frac{c^{2}}{a^{2}}\right)^{1 / 2}=\left(\xi_{0}^{2}+1\right)^{-1 / 2}
$$

The ratio of the total cross-section to the surface area is

$$
\frac{\sigma}{A}=\frac{2}{\left(\arctan \left(1 / \xi_{0}\right)\right)^{2}} \frac{1}{\xi_{0}^{2}+1+\frac{\xi_{0}^{2}}{2 e} \ln \frac{1+e}{1-e}} .
$$

In the spherical limit $\left(\xi_{0} \rightarrow \infty, e \rightarrow 0\right)$ the ratio of Eqn. 4.54 is unity, whereas in the disk limit $\left(\xi_{0} \rightarrow 0, e \rightarrow 1^{-}\right)$the ratio is $8 / \pi^{2} \approx 0.81$. Fig. 4.2 shows this ratio plotted as a function of $\alpha=\arctan \left(1 / \xi_{0}\right)$, which varies from zero to $\pi / 2$. In terms of the angle $\alpha$, the total cross-section to surface area ratio is

$$
\frac{\sigma}{A}=\frac{2 \sin ^{3} \alpha}{\alpha^{2}\left[\sin \alpha+\cos ^{2} \alpha \ln \left(\frac{1+\sin \alpha}{\cos \alpha}\right)\right]} .
$$

This function is remarkably flat for small $\alpha$ (which corresponds to large $\xi_{0}=\cot \alpha$, and nearly spherical scatterers):

$$
\frac{\sigma}{A}=1-\frac{4}{945} \alpha^{6}+O\left(\alpha^{8}\right)
$$

In the opposite limit, where $\alpha$ is near $\pi / 2$ (which corresponds to small $\xi_{0}=$ $\cot \alpha$, and nearly disk shaped scatterers) the ratio of the total cross-section to the surface area is

$$
\frac{\sigma}{A}=\frac{8}{\pi^{2}}-\frac{32}{\pi^{3}}\left(\alpha-\frac{1}{2} \pi\right)+O\left(\left(\alpha-\frac{1}{2} \pi\right)^{2}\right) .
$$




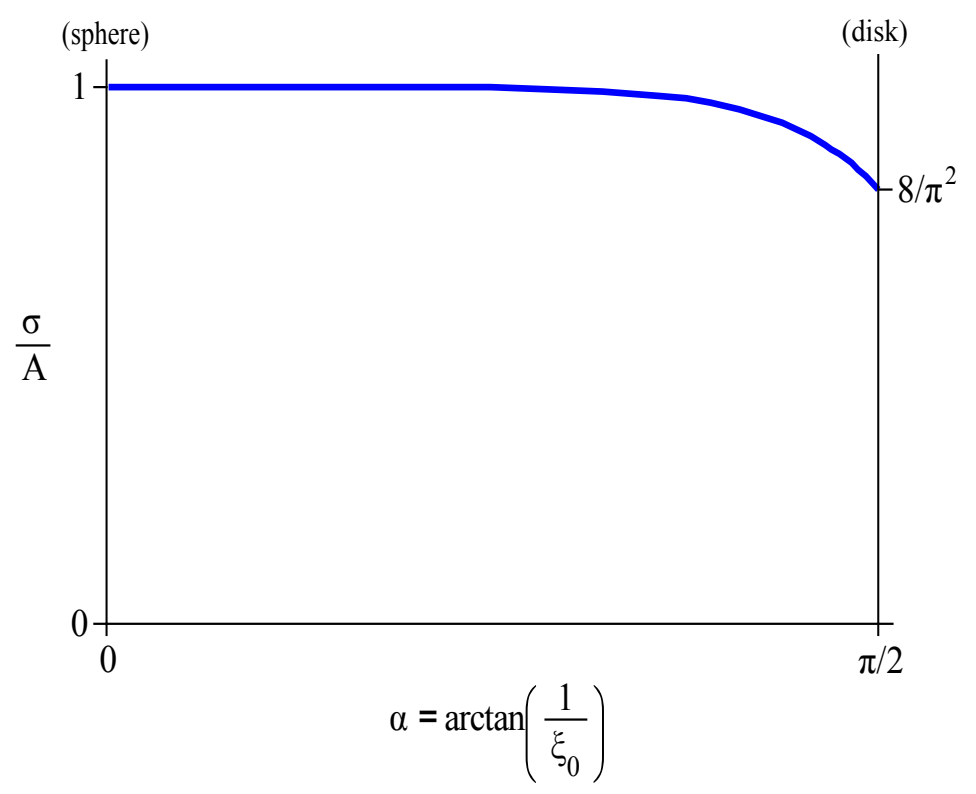

Figure 4.2: The ratio of the total cross-section to the surface area of an oblate spheroid, in the long-wavelength limit.

Fig. 4.2 shows the weak divergence of the ratio of $\sigma / A$ from unity to the disk value. For nearly spherical scatterers the total-cross section to surface area ratio is very close to unity, however, in the limit of the disk this ratio is $\left(8 / \pi^{2}\right)$.

\subsection{Scattering by prolate spheroids}

We may also investigate prolate spheroids by applying the Legendre transform to the prolate version of Helmholtz's equation. The prolate spheroidal coordinate system is defined by

$$
\begin{array}{r}
\rho=b \sqrt{\xi^{2}-1} \sqrt{1-\eta^{2}}, \quad z=b \xi \eta, \quad \phi=\phi, \\
-1 \leq \eta \leq 1, \quad 1 \leq \xi<\infty, \quad 0 \leq \phi \leq 2 \pi .
\end{array}
$$

The surface $\xi=\xi_{0}$ is an ellipsoid with semi axes $a, a, c$ where $a=b\left(\xi_{0}^{2}-1\right)^{1 / 2}$, $c=b \xi_{0}$ :

$$
\frac{\rho^{2}}{b^{2}\left(\xi_{0}^{2}-1\right)}+\frac{z^{2}}{b^{2} \xi_{0}^{2}}=1
$$


The surface $\eta=\eta_{0}$ is a two-sheet hyperboloid:

$$
\frac{\rho^{2}}{b^{2}\left(1-\eta_{0}^{2}\right)}-\frac{z^{2}}{b^{2} \eta_{0}^{2}}=-1
$$

We again consider scattering of a plane wave $e^{i k z}$ incident along the symmetry axis of the prolate spheroid. The Helmholtz equation then has no $\phi$-dependence, and reads

$$
\left\{\partial_{\xi}\left(\xi^{2}-1\right) \partial_{\xi}+\partial_{\eta}\left(1-\eta^{2}\right) \partial_{\eta}+\beta^{2}\left(\xi^{2}-\eta^{2}\right)\right\} \psi(\xi, \eta)=0 .
$$

Instead of separating the variables we again apply the Legendre transform Eqn. 4.13; that is, we operate on Eqn. 4.61 with $\frac{1}{2} \int_{-1}^{1} P_{n}(\eta) d \eta$. As in the oblate case, the result is a difference-differential equation for the radial functions $R_{n}(\xi)=\frac{1}{2} \int_{-1}^{1} \psi(\xi, \eta) P_{n}(\eta) d \eta$ :

$$
\begin{aligned}
& {\left[\partial_{\xi}\left(\xi^{2}-1\right) \partial_{\xi}+\beta^{2} \xi^{2}-n(n+1)\right] R_{n}(\xi) } \\
- & \frac{\beta^{2}}{2 n+1}\left\{\frac{n(n-1)}{2 n-1} R_{n-2}(\xi)+\left[\frac{(n+1)^{2}}{2 n+3}+\frac{n^{2}}{2 n-1}\right] R_{n}(\xi)\right. \\
+ & \left.\frac{(n+1)(n+2)}{2 n+3} R_{n+2}(\xi)\right\}=0 .
\end{aligned}
$$

Solutions of Eqn. 4.62 are $R_{n}(\xi)=P_{n}(\xi)$ from $n=0$, and $Q_{n}(\xi)$ from $n=2$. In the low-frequency limit, $\beta=k b \rightarrow 0$, the solutions

$$
R_{n}(\xi)=p_{n} P_{n}(\xi)+q_{n} Q_{n}(\xi)
$$

are valid at $n=0$ and $n=1$. Thus the dominant $n=0$ partial wave radial function is a linear combination of $P_{0}(\xi)=1$ and $Q_{0}(\xi)=\frac{1}{2} \ln \left(\frac{\xi+1}{\xi-1}\right)$. For the acoustically soft scatterer, and the quantum impenetrable scatterer, the $n=0$ solution at long-wavelengths satisfying $R_{0}\left(\xi_{0}\right)=0$ is thus

$$
R_{0}(\xi)=\ln \left(\frac{\xi_{0}+1}{\xi_{0}-1}\right)-\ln \left(\frac{\xi+1}{\xi-1}\right) .
$$

All of the formulation of scattering by oblate spheroids translates to the prolate case, including the plane-wave expansion Eqn. 4.31. Thus we can apply Eqn. 4.45 to Eqn. 4.64 to extract the scattering length $s=b \xi_{s}$. This gives

$$
\xi R_{0}(\xi) \rightarrow \xi \ln \left(\frac{\xi_{0}+1}{\xi_{0}-1}\right)-2+O\left(\xi^{-2}\right)
$$


Thus, in agreement with the known results for low-frequency scattering [22]

$$
\xi_{s}=\frac{2}{\ln \left(\frac{\xi_{0}+1}{\xi_{0}-1}\right)} .
$$

The long-wavelength limit of the total cross-section is therefore

$$
\sigma=4 \pi s^{2}=\frac{16 \pi b^{2}}{\left[\ln \left(\frac{\xi_{0}+1}{\xi_{0}-1}\right)\right]^{2}} .
$$

The surface area of a prolate spheroid (semi axes $a, a, c)$ is

$$
A=2 \pi a^{2}\left(1+\frac{\gamma}{\sin \gamma \cos \gamma}\right), \quad \gamma=\arccos \left(\frac{a}{c}\right) .
$$

Here $a=b\left(\xi_{0}^{2}-1\right)^{1 / 2}, c=b \xi_{0}$. In the spherical limit $\left(b \xi_{0}>>1\right)$ the surface area tends to $4 \pi\left(b \xi_{0}\right)^{2}, b \xi_{0}$ being the radius of the sphere. The total crosssection also tends to $4 \pi\left(b \xi_{0}\right)^{2}$, in a similar way to Eqn. 4.56 :

$$
\frac{\sigma}{A}=1+\frac{4}{945} \xi_{0}^{-6}+O\left(\xi_{0}^{-8}\right) .
$$

The opposite extreme $\left(\xi_{0} \rightarrow 1+\right)$ is end-on scattering by a needle-shaped object. In this limit the 'wrap around' idea of long-wave scattering fails, since $\sigma / A$ diverges, though weakly:

$$
\frac{\sigma}{A} \rightarrow \frac{8 \sqrt{2} / \pi}{\left[\ln \frac{2}{\xi_{0}-1}\right]^{2}\left(\xi_{0}-1\right)^{1 / 2}}+O\left(\xi_{0}-1\right)^{1 / 2} .
$$

In terms of the angle $\gamma=\arccos (a / c)=\arcsin \left(1 / \xi_{0}\right)$, the ratio of the crosssection to the spheroid surface area is

$$
\begin{aligned}
\frac{\sigma}{A} & =\frac{8 \sin ^{3} \gamma}{\left[\ln \left(\frac{1+\sin \gamma}{1-\sin \gamma}\right)\right]^{2} \cos \gamma[\sin \gamma \cos \gamma+\gamma]}, \\
& =1+\frac{4}{945} \gamma^{6}+O\left(\gamma^{8}\right) .
\end{aligned}
$$

Fig. 3 shows that $\sigma \approx A$ is a very good approximation for most of the range of the angle $\gamma$. 


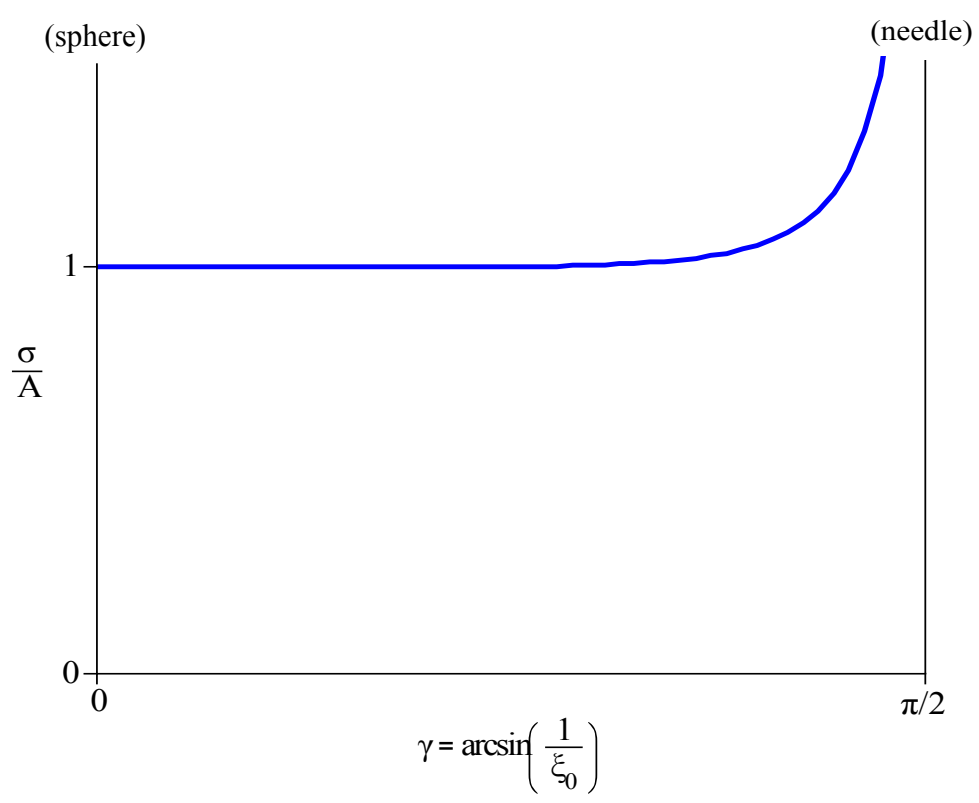

Figure 4.3: Ratio of the long-wavelength scattering cross-section to the surface area of a prolate spheroid.

The analysis performed here was for scattering of scalar waves by spheroids. For discussion of scattering of electromagnetic waves, by oblate and prolate spheroids, in the long-wavelength limit, known as the electrostatics approximation, see Chapter 5 of [68]. The general electromagnetic theory of scattering by spheroidal particles, using the vector spheroidal wave functions, in a form similar to Mie theory, has been carried out by Asano and Yamamoto [69, 70].

In conclusion we have presented an alternative treatment of the scattering of scalar waves by spheroids, which results in a one-to-one correspondence with the partial wave analysis of scattering by spherical objects. The only restriction is that the wave is incident on the scatterer along its symmetry axis. Long-wavelength scattering by acoustically soft spheroids follows from the spheroidal analogue of a scattering length, which has been obtained analytically. 


\section{Chapter 5}

\section{Discussion and Conclusions}

In this final Chapter the main results of Chapters 2-4 shall be presented, with a discussion of possible areas for future research.

\section{Chapter 2: The hydrogen molecule ion}

Chapter 2 investigated the ground state and certain excited states of the hydrogen molecule ion. The main achievement in this section was the derivation of the Wronskian method. In particular we showed the following:

- The angular Wronskian gives an exact transcendental expression whose roots give all the physical separation parameters.

As the Heun functions are implemented in Maple, the Wronskian allows all the physical separation parameters of the spheroidal wave equation to be computed, with unlimited precision. There are a number of other applications using spheroidal wave functions, such as scattering by spheroids, which require the separation parameter to be accurately known. The Wronskian result is therefore of benefit to applications involving spheroidal wave functions where the separation parameter must be known over a wide range of its variable. 


\section{Chapter 3: Scalar and Electromagnetic beams}

Chapter 3 investigated scalar beam solutions of the Helmholtz equation. Initially exact beam wave functions in terms of confluent Heun functions, and then subsequently in terms of oblate spheroidal wave functions, were studied. The results obtained concerning oblate spheroidal wave functions were the following:

- The oblate spheroidal wave functions may only represent physical free space scalar beams when $n-m$ is odd. This allows for the physically necessary discontinuity in phase outside the circle $\rho=b$ in the focal plane on the ellipsoidal surfaces of otherwise constant phase.

- If $n-m$ is odd the oblate spheroidal wave functions are normalizable in any cross-section of the beam.

- The oblate spheroidal wave functions are manifestly forward propagating.

- The $n-m$ odd condition leads to a wave function with a discontinuous $z$ derivative, in the entire $z=0$ plane.

- The oblate spheroidal wave functions are not physical free space scalar or (TM and TE) electromagnetic beams. More generally, no simple product solution $\psi(\xi, \eta, \phi)=R(\xi) S(\eta) \Phi(\phi)$ can represent a physical free space scalar beam.

The oblate spheroidal wave functions have been shown to require $n-m$ odd. However, this leads to a discontinuous $\rho$ derivative across the boundary $\xi=\eta=0$. Furthermore, when $\eta=0$, where $\psi_{m n}(\xi, \eta)=0$, we showed that the $z$ derivative of $\psi$ is infinite as $\xi \rightarrow 0$. Any physical quantum particle beam must have a continuous wave function and a continuous derivative at all points in space. The associated divergence of the ( $\rho$ and $z$ ) derivatives of the oblate spheroidal wave functions (when $n-m$ is odd) indicates they are not physical free space scalar beams. Therefore, the problem of deriving exact solutions of the Helmholtz equation which represent physical free scalar beams remains. 


\section{Chapter 4: Scattering of scalar waves by sphe- roids}

The scattering of Schrödinger particle waves by quantum-mechanically hard oblate and prolate spheroids was investigated in Chapter 4. In analogy with the partial wave theory of scattering by spherical scatterers, a general formulation of the scattering by spheroids was formulated. In particular we showed that

- The radial eigenfunctions, for axial scattering by spheroidal obstacles, solve certain difference-differential equations.

- The spheroidal scattering theory has a one-to-one correspondence with the spherical scattering theory.

- The exact solutions in the long-wavelength limit, subject to axially symmetric incidence, are linear combinations of the singular and nonsingular Legendre functions.

Scattering by spherical objects, outside the range of influence of the scatterer, uses known free space radial functions the spherical Bessel functions. In the spheroidal case the corresponding functions are solutions of difference-differential equations. Although we have found exact solutions of these equations they are not the general solutions, and are physical only in the long-wavelength limit.

\section{Possible future research}

Finding an exact solution of the radial equation which is linearly dependent with $X_{1}(\xi)$, when $R=R(\lambda)$, would enable a radial Wronskian to determine the $R(\lambda)$ and $\lambda$ relation exactly. Two solutions to the radial equation were derived, however, they were shown to be linearly independent. Further investigation into finding another solution to the radial equation, which is linearly dependent with $X_{1}(\xi)$, when $R=R(\lambda)$, may allow a complete solution to the problem. 
For the radial equation arising from the Helmholtz equation, we showed if $K(\xi, \eta)$ is any solution, independent of the azimuthal angle $\phi$, of the Helmholtz equation, $X(\xi)=\int_{-1}^{1} K(\xi, \eta) Y(\eta) d \eta$ will satisfy the radial equation provided $Y(\eta)$ satisfies the angular equation (both having the same $\mu(\beta)$ separation constant, of course).

One can do the same for the $\mathrm{H}_{2}^{+}$problem, up to a point: define $L_{\eta}$ as before by Eqn. 3.52 with $\lambda=\beta^{2}$, and

$$
L_{\xi}=\partial_{\xi}\left(\xi^{2}-1\right) \partial_{\xi}+2 R \xi-\lambda \xi^{2}
$$

and let $K(\xi, \eta)$ be any solution (independent of $\phi$ ) of the Schrödinger equation, i.e. satisfying $\left(L_{\xi}+L_{\eta}\right) K(\xi, \eta)=0$. Then it follows as before that the integral $\int_{-1}^{1}\left[Y L_{\eta} K-K L_{\eta} Y\right] d \eta$ is zero, and thus if $\left(L_{\eta}-\mu\right) Y=0$,

$$
X(\xi)=\int_{-1}^{1} K(\xi, \eta) Y(\eta) d \eta
$$

will solve the radial equation $\left(L_{\xi}+\mu\right) X=0$. This might be useful in determining the asymptotic form of $X(\xi)$, and thus the relationship between $\lambda$ and the internuclear separation $R$, if a suitable solution of the Schrödinger equation could be found.

Most of the beam solutions considered in Chapter 3 have had some nonphysical aspect to them. The confluent Heun beams are not physical due to a divergent normalization integral and backward propagating elements. The spherical Bessel beams are not physical due to backward propagating elements. The oblate spheroidal beams, with $n-m$ odd, have a discontinuous $z$ derivative, in the entire $z=0$ plane. Only the generalized Bessel beams are physical scalar beams. An area of future research would be to determine what weight function $(\mathrm{s}) f(\kappa)$ lead to analytic expressions of physical scalar, and electromagnetic, free space beams.

The solution of the difference-differential equations for the radial eigenfunctions would allow the spheroidal scattering theory constructed to be fully functional. This would enable the theory to be able to do for axial scattering by spheroidal bodies all that can be done by the phase-shift theory for centrally symmetric scatterers. Thus determining the radial eigenfunctions would be an aspect to look at in the future. 


\section{Appendix A}

\section{The Heun equation}

\section{A.1 General Heun equation}

The Heun equation [71] is a Fuchsian linear second-order ordinary differential equation. It is the natural generalization of the hypergeometric differential equation, which has three regular singularities. The Heun equation is an extension of the hypergeometric differential equation in that it has four regular singularities. The Heun equation may also be interpreted as a confluent form of the differential equation with eight elementary singularities, the singularities being combined in pairs to form four regular singularities, but little is known about this differential equation. The canonical form of the general Heun equation (GHE) is given by

$$
\frac{d^{2} y}{d z^{2}}+\left\{\frac{\gamma}{z}+\frac{\delta}{z-1}+\frac{\epsilon}{z-a}\right\} \frac{d y}{d z}+\frac{\alpha \beta z-q}{z(z-1)(z-a)} y=0 .
$$

Eqn. A.1 is essentially due to Heun himself, however, Heun had $\alpha \beta(z-q)$ as the coefficient of $y$. The form in Eqn. A.1 allows $\alpha=0$, or $\beta=0$, without eliminating the term in $y$. In this form $y$ and $z$ are both complex variables, $\alpha, \beta, \gamma, \delta, \epsilon, q, a$ are arbitrary parameters, generally complex, except $a \neq 0,1$. The equation has regular singularities at $z=0,1, a, \infty$, with the characteristic (or indicial) exponents at these singularities given by $\{0,1-\gamma\} ;\{0,1-\delta\} ;\{0,1-\epsilon\} ;\{\alpha, \beta\}$. 
The sum of these exponents is equal to two as a result of Fuch's theorem; that is

$$
\gamma+\delta+\epsilon=\alpha+\beta+1
$$

From the characteristic exponents the Riemann P symbol of the Heun equation follows

$$
P\left(\begin{array}{cccccc}
0 & 1 & a & \infty & & \\
0 & 0 & 0 & \alpha & z & q \\
1-\gamma & 1-\delta & 1-\epsilon & \beta & &
\end{array}\right)
$$

The parameter $a$ locates the third finite singularity and is called the singularity parameter, while $\alpha, \beta, \gamma, \delta, \epsilon$ determine the exponents at the four singularities; the other parameter $q$ is called the accessory parameter.

It should be mentioned that in Eqn. A.1 one of the four regular singularities has been taken to be infinity. This is without any loss of generality, since any Fuchsian second-order equation with four arbitrary finite regular singularities can be transformed into this form by suitable transformations, see Chapter 2, section 3 of [35]. Of course, this assumes that infinity is an ordinary point of the original equation. In fact, any Fuchsian second-order differential equation with four (three finite and one infinite, or four finite) regular singularities can be transformed into the Heun equation.

Since any equation with three regular singularities can be transformed into a hypergeometric differential equation, any equation with three regular singularities can be transformed into a Heun equation too. These few remarks presented here should already indicate the scope of the Heun equation and why it is of such interest, from both a pure and applied point of view.

The differential equations of classical physics: Bessel, Legendre, Laguerre, etc can all be represented in the form of hypergeometric (or confluent hypergeometric) differential equations, but now many problems arising in quantum mechanics must be represented in terms of Heun equations. The hydrogen molecule ion is the most pertinent example in this case. Other quantum mechanical applications may be found in Chapter 4 of [34]. 
It is interesting to consider the solutions to the general Heun equation Eqn. A.1. The Heun equation may be transformed into another version of itself; that is, an equation with four regular singularities, three of which are located at $z=0,1, \infty$, by any of twenty four Möbius transformations $z \rightarrow f(z)$. The Heun equation has four regular singularities, and around each singularity it is possible to construct two local Frobenius solutions. Since there are twenty four transformations which leave the form of the Heun equation unaltered, therefore, there are 192 possible solutions to the general Heun equation [72]. This is analogous to Kummer's 24 solutions of the hypergeometric differential equation.

One such solution is the function $\operatorname{HeunG}(a, q, \alpha, \beta, \gamma, \delta, z)$ (in Maple notation), which satisfies the conditions at the origin $y(0)=1, y^{\prime}(0)=q / \gamma a$. The function HeunG $(a, q, \alpha, \beta, \gamma, \delta, z)$ is a local Frobenius solution of the GHE, computed as a power series about the regular singular point $z=0$. By the theory of local Frobenius solutions, the function $\operatorname{HeunG}(a, q, \alpha, \beta, \gamma, \delta, z)$ has a radius of convergence $\min (1,|a|)$, i.e. out to the location of the nearest singularity.

In certain cases it is possible that the local Frobenius solution is also valid about the nearest adjacent singularity. These solutions are known as Heun functions, and are analytic in a domain containing both singularities, see Chapter 3 of [35].

A third type of solution are Heun polynomials [73]. Heun polynomials are, by definition, a local Frobenius solution of the Heun equation, valid at three singularities. A Heun polynomial is thus defined in the whole plane, except at the singularities, and with certain cuts for single-valuedness.

The wide scope of the Heun equation lies in its various confluent forms. Confluence is a process whereby two singularities in a differential equation are brought into coincidence. It arises as a result of a suitable limiting procedure in the Laurent expansion of the coefficient functions of the differential equation, so that the two singularities merge into one singularity, generally of a more complicated nature. There are four confluent forms of the Heun equation. These are the confluent Heun equation, the doubly confluent Heun equation, the biconfluent Heun equation, and the triconfluent Heun equation. 
A summary of these equations shall be presented next, with emphasis on the confluent Heun equation due to its relation to the hydrogen molecule ion, and scalar beam problems.

\section{A.2 Confluent Heun equation}

The Heun equation of most importance in this thesis was the confluent Heun equation (CHE). The CHE arises from the coalescence of the two regular singularities at $z=a$ and $z=\infty$ in the GHE. This process results in an irregular singularity at $z=\infty$. The coalescence of two regular singularities typically (although there are exceptions) resulting in an irregular singularity, see the Addendum of [35]. For an elucidation of the coalescence process we derive the CHE from the GHE. The GHE is given in Eqn. A.1, suppose we redefine the parameters as follows

$$
\beta=\beta a, \quad \epsilon=\epsilon a, \quad q=q a .
$$

Now take the limit $a \rightarrow \infty$, the resulting equation is

$$
\frac{d^{2} y}{d z^{2}}+\left\{\frac{\gamma}{z}+\frac{\delta}{z-1}-\epsilon\right\} \frac{d y}{d z}-\left\{\frac{\alpha \beta-q}{z-1}+\frac{q}{z}\right\} y=0 .
$$

Eqn. A.5 is a (non-symmetrical) CHE, which has two regular singularities at $z=0$ and $z=1$, and an irregular singularity at $z=\infty$. (The CHE is symmetrical if the two regular singularities are at $z=-1$ and $z=1$.) $\mathrm{A}$ canonical form of the CHE, with regular singularities at $z=0$ and $z=1$, and an irregular singularity at $z=\infty$ is

$$
\begin{aligned}
& \frac{d^{2} y}{d z^{2}}+\left\{\alpha+\frac{\beta+1}{z}+\frac{\gamma+1}{z-1}\right\} \frac{d y}{d z}+\left\{\frac{\nu}{z-1}+\frac{\mu}{z}\right\} y=0, \\
\text { where } \mu= & \frac{1}{2}[\alpha-\beta-\gamma-2 \epsilon+\beta(\alpha-\gamma)], \\
\nu= & \frac{1}{2}[\alpha+\beta+\gamma+2 \delta+2 \epsilon+\gamma(\alpha+\beta)] .
\end{aligned}
$$


The standard confluent Heun function, as implemented in maple, is the unique solution of Eqn. A.6, regular at $z=0$ and normalized to unity at the origin:

$$
\operatorname{HeunC}(\alpha, \beta, \gamma, \delta, \epsilon, z)=\sum_{n=0}^{\infty} A_{n}(\alpha, \beta, \gamma, \delta, \epsilon) z^{n}, \quad|z|<1,
$$

where the coefficients $A_{n}$, typically, satisfy three term recurrence relations. A second linearly independent solution is given by $z^{-\beta} \operatorname{HeunC}(\alpha,-\beta, \gamma, \delta, \epsilon, z)$. However, when $\beta$ is an integer these two solutions are not independent. By considering the invariance under the transformation $z \rightarrow 1-z$, a second pair of independent solutions is given by $\operatorname{HeunC}(-\alpha, \gamma, \beta,-\delta, \epsilon+\delta, 1-z)+(z-$ $1)^{-\gamma}$ HeunC $(-\alpha,-\gamma, \beta,-\delta, \epsilon+\delta, 1-z)$. This invariance has been explored in the context of the solutions to the angular equation in the hydrogen molecule ion, and scalar beam problems, see Eqn. 2.41.

The CHE has two regular singularities and one irregular singularity. It includes as particular cases both the ${ }_{2} \mathrm{~F}_{1}$ and ${ }_{1} \mathrm{~F}_{1}$ hypergeometric equations. An important non-hypergeometric case of the CHE is the spheroidal wave equation, which has been discussed in section 3.6.2. The spheroidal wave equation is given by the following equation

$$
\left(z^{2}-1\right) \frac{d^{2} y}{d z^{2}}+2(\gamma+1) z \frac{d y}{d z}+\left(4 \delta z^{2}-c\right) y=0,
$$

which is obtained from the CHE by taking $\alpha=0, \beta=-1 / 2, \epsilon=(1-\gamma-c) / 4$ and changing $z \rightarrow z^{2}$. Another important non-hypergeometric case of the CHE is the Mathieu equation, which arises from the separation of variables of the Helmholtz differential equation in elliptic cylindrical coordinates. The rational form of the Mathieu equation is given by

$$
\left(z^{2}-1\right) \frac{d^{2} y}{d z^{2}}+z \frac{d y}{d z}+\left[2 \delta\left(2 z^{2}-1\right)-a\right] y=0,
$$

which is obtained from the spheroidal wave equation by taking $c=a+2 \delta$ and $\gamma=-1 / 2$. Eqn. A.9 has two regular singularities at $z=-1$ and $z=1$, and an irregular singularity at $z=\infty$. Thus it is not expressible in terms of hypergeometric functions, however, it is solvable in terms of the HeunC function HeunC $\left(0,-\frac{1}{2},-\frac{1}{2}, \delta, \frac{3}{8}-\frac{1}{2} \delta-\frac{1}{4} a, z^{2}\right)$. 
Physical applications utilizing the confluent Heun equation are numerous. Exton [74] has shown that the Schrödinger equation with interaction $V(r)=$ $-Z e^{2} /(r+\beta)$ is reducible to a confluent Heun equation, for general values of the parameters. El-Jaick and Figueiredo [48] have also investigated the Schrödinger equation for an inverted potential and for an electric dipole. The paper by El-Jaick and Figuieredo considers solutions in terms of series of Bessel functions, which are related to the spherical Bessel expansions derived in section 3.4. There are also areas in general relativity, and gravitational physics, where confluent Heun equations and confluent Heun functions play an important role, see [73] and the references therein. For further applications of confluent Heun equations see Chapter 4 of the book by Slavyanov and Lay [34].

\section{A.3 Double confluent Heun equation}

Section A.2 considered the confluent Heun equation, which arose from the coalescence of two regular singularities in the general Heun equation. Similarly we may consider the coalescence of the two regular singularities in the CHE, which gives rise to an equation with two irregular singularities, located at $z=0$ and $z=\infty$. This is known as the double confluent Heun equation (DCHE). The DCHE may be further transformed, relocating the singularities to $z=-1$ and $z=1$. This DCHE is given by

$$
\frac{d^{2} y}{d z^{2}}-\frac{\left(-2 z^{3}+z^{2} \alpha+2 z+\alpha\right)}{(z-1)^{2}(z+1)^{2}} \frac{d y}{d z}+\frac{\left(z^{2} \beta+(\gamma+2 \alpha) z+\delta\right)}{(z-1)^{3}(z+1)^{3}} y=0 .
$$

The function HeunD is the solution of Eqn. A.10. The conditions satisfied by HeunD at the origin are $y(0)=1, y^{\prime}(0)=0$. The function $\operatorname{HeunD}(\alpha, \beta, \gamma, \delta, z)$ is a local solution to the DCHE, computed as a standard power series expansion about the origin. The radius of convergence of this local Frobenius solution is $|z|<1$, due to the irregular singularities located at $|z|=1$. Analytic continuation of the HeunD function outside the unit circle is obtained through the identity $\operatorname{HeunD}(\alpha, \beta, \gamma, \delta, z)=$ $\operatorname{HeunD}(-\alpha,-\delta,-\gamma,-\beta, 1 / z)$. 


\section{A.4 Biconfluent Heun equation}

The coalescence of the two regular singularities in the CHE leads to an equation (DCHE) with two irregular singularities. However, we may combine the irregular singularity with one of the regular singularities in the CHE, which leads to an equation with one regular and one irregular singularity. This is the biconfluent Heun equation (BHE). The BHE has a regular singularity at $z=0$, and an irregular singularity at $z=\infty$. The BHE is

$\frac{d^{2} y}{d z^{2}}-\left\{\frac{2 z^{2}-1-\alpha+\beta z}{z}\right\} \frac{d y}{d z}-\frac{1}{2}\left\{\frac{(-2 \gamma+2 \alpha+4) z+\delta+\beta+\beta \alpha}{z}\right\} y=0$.

The function $\operatorname{HeunB}(\alpha, \beta, \gamma, \delta, z)$ is a local Frobenius solution to Eqn. A.11, computed as a power series expansion about the origin. Because the next singularity is located at infinity this series converges in the whole complex plane. The HeunB function satisfies the conditions $y(0)=1, y^{\prime}(0)=(\delta+$ $\alpha \beta+\beta) /(2 \alpha+2)$ at the origin.

\section{A.5 Triconfluent Heun equation}

The triconfluent Heun equation (THE) arises as a result of the coalescence of three regular singularities in the GHE. (The THE must, however, be derived from the GHE written in the special form where the singularities are located at $a_{1}, a_{2}, a_{3}, \infty$, and let $a_{1} \rightarrow a_{2} \rightarrow a_{3} \rightarrow \infty$. The resulting equation has an irregular singularity at $z=\infty$, of rank three [35], with no finite singularities at all. The THE is given by

$$
\frac{d^{2} y}{d z^{2}}-\left(3 z^{2}+\gamma\right) \frac{d y}{d z}-[(3-\beta) z-\alpha] y=0 .
$$

The function HeunT is a solution of Eqn. A.12, which satisfies the following conditions at the origin, $y(0)=1, y^{\prime}(0)=0$. The function $\operatorname{HeunT}(\alpha, \beta, \gamma, z)$ is a local Frobenius solution to the THE, computed as a power series expansion about the origin. Because the nearest (irregular) singularity occurs at $z=\infty$, this local Frobenius solution is convergent in the whole complex plane. 


\section{Appendix B}

\section{Abstracts of papers}

OPTICS LETTERS / Vol. 35, No. 21 / November 1, 2010

\section{Constraints on spheroidal beam wavefunctions}

\section{John Lekner* and Rufus Boyack}

MacDiarmid Institute for Advanced Materials and Technology, and School of Chemical and Physical Sciences, Victoria University of Wellington, P.O. Box 600, Wellington, New Zealand "Corresponding author: john.lekner@vuw.ac.nz

Received August 10, 2010; revised September 27, 2010; accepted September 28, 2010; posted October 4, 2010 (Doc. ID 132829); published October 26, 2010

The oblate spheroidal wavefunctions proposed by Rodríguez-Morales and Chávez-Cerda are shown to be possible representations of physical beams only when the angular function $S_{m n}(\beta, \eta)$ has odd $n-m$. This condition makes $S_{m u}$ odd in $\eta$, which ensures the convergence of integrals of physical quantities over a cross section of the beam. The odd $n-m$ condition also makes $S_{m n}(\beta, \eta)$ zero in the focal plane $z=0$ outside the circle $\rho=b$, and thus allows for the physically necessary discontinuity in phase at $z=0$ on the ellipsoidal surfaces of otherwise constant phase. Only a subset of the oblate spheroidal functions can be exact representations of nonparaxial scalar beams. 12010 Optical Society of America

OCIS codes: $\quad 350.5500,260.1960,260.2110,140.3410,000.3860$

Figure B.1: Constraints on spheroidal beam wavefunctions.

\section{Non-existence of separable spheroidal beams}

We show that $\psi=R(\xi) S(\eta) e^{i m \phi}$, a product of radial and angular oblate spheroidal functions and an azimuthal factor, cannot represent physical scalar beams. The reason lies in the discontinuity in the longitudinal derivative of $\psi$ in the focal plane, where $\psi$ is not a solution of the Helmholtz equation on the disk $\xi=0$.

In preparation 


\section{Axisymmetric scattering of scalar waves by spheroids}

We give a phase shift formulation of scattering by oblate and prolate spheroids, in parallel with the partial-wave theory of scattering by spherical obstacles. The crucial step is application of a finite Legendre transform to the Helmholtz equation in spheroidal coordinates. In the long-wavelength limit the spheroidal analogue of the spherical scattering length immediately gives the cross-section. Analytical results are readily obtained for scattering of Schrödinger particle waves by impenetrable spheroids, and for scattering of sound waves by acoustically soft spheroidal objects. The method is restricted to scattering by spheroids whose symmetry axis is coincident with the direction of the incident plane wave.

Submitted to J. Acoust. Soc. Am.

\section{Properties of confluent Heun functions rele- vant to the Helmholtz equation and to Schrö- dinger's equation for $\mathbf{H}_{2}^{+}$}

The Helmholtz equation and Schrödinger's equation for $\mathrm{H}_{2}^{+}$are separable in (respectively) oblate and prolate spheroidal coordinates. They share the same form of the angular equation. In both cases the radial and angular equations have solutions in terms of confluent Heun functions. We show that the zeros of the Wronskian of a pair of solutions to the angular equation give the allowed values of the separation of variables parameter. We also derive the asymptotic forms of the radial solutions of the Helmholtz equation, and obtain integral relations between the radial and angular solutions.

Submitted to J. Math. Phys. 


\section{Bibliography}

[1] W. Pauli Ann. d. Phys., vol. 68, 1922.

[2] K. F. Niessen, "Zur Quantentheorie des Wasserstoffmolekülions," Dissertation, Utrecht, 1922.

[3] O. Burrau, "Berechnung des Energiewertes des Wasserstoffmolekelions im normalzustand," Det Kgl. Danske Videnskabernes Selskab. Mathematisk-fysiske Meddelelser., vol. VII, no. 14, pp. 2-18, 1927.

[4] A. H. Wilson, "The Ionised Hydrogen Molecule," Proc. Roy. Soc. London. Series A, vol. 118, no. 780, pp. 635-647, 1928.

[5] A. H. Wilson, "A Generalised Spheroidal Wave Equation," Proc. Roy. Soc. London. Series A, vol. 118, no. 780, pp. 617-635, 1928.

[6] G. Jaffé, "Zur Theorie des Wasserstoffmolekülions," Zeitschrift für Physik, vol. 87, pp. 535-544, 1934.

[7] E. A. Hylleraas, "Über die Elektronenterme des Wasserstoffmoleküls," Zeitschrift für Physik, vol. 71, pp. 739-763, 1931.

[8] L. Pauling and E. B. Wilson, Introduction to Quantum Mechanics With Applications to Chemistry. New York: McGraw-Hill, 1935.

[9] L. Pauling, "The Application of the Quantum Mechanics to the Structure of the Hydrogen Molecule and Hydrogen Molecule-Ion and to Related Problems," Chemical Reviews, vol. 5, pp. 173-213, 1928.

[10] M. Lax, W. H. Louisell, and W. B. McKnight, "From Maxwell to paraxial wave optics," Phys. Rev. A, vol. 11, no. 4, pp. 1365-1370, 1975. 
[11] G. A. Deschamps, "Gaussian beam as a bundle of complex rays," Electronics Letters, vol. 7, no. 23, pp. 684-685, 1971.

[12] C. J. R. Sheppard and S. Saghafi, "Beam modes beyond the paraxial approximation: A scalar treatment," Phys. Rev. A, vol. 57, no. 4, pp. 2971-2979, 1998.

[13] C. J. R. Sheppard and S. Saghafi, "Electromagnetic Gaussian beams beyond the paraxial approximation," J. Opt. Soc. Am. A, vol. 16, pp. 1381-1386, 1999.

[14] C. J. R. Sheppard, "High-aperture beams: reply to comment," J. Opt. Soc. Am. A, vol. 24, no. 4, pp. 1211-1213, 2007.

[15] Z. Ulanowski and I. K. Ludlow, "Scalar field of nonparaxial Gaussian beams," Opt. Lett., vol. 25, no. 24, pp. 1792-1794, 2000.

[16] J. Lekner, "TM, TE and 'TEM' beam modes: exact solutions and their problems," J. Opt. A:Pure Appl. Opt., vol. 3, pp. 407-412, 2001.

[17] G. Rodríguez-Morales and S. Chávez-Cerda, "Exact nonparaxial beams of the scalar Helmholtz equation," Opt. Lett., vol. 29, no. 5, pp. 430-432, 2004 .

[18] J. Lekner and R. Boyack, "Constraints on spheroidal beam wavefunctions," Opt. Lett., vol. 35, no. 21, pp. 3652-3654, 2010.

[19] J. W. S. Rayleigh, "On the Passage of Waves through Apertures in Plane Screens, and Allied Problems," Phil. Mag., vol. 43, pp. 259-272, 1897.

[20] R. D. Spence and S. Granger, "The Scattering of Sound from a Prolate Spheroid," J. Acoust. Soc. Am., vol. 23, no. 6, pp. 701-706, 1951.

[21] T. B. A. Senior, "The Scattering from Acoustically Hard and Soft Prolate Spheroids for Axial Incidence," Can. J. Phys., vol. 44, no. 3, pp. 655-667, 1966. 
[22] T. B. A. Senior, "Low-frequency scattering," J. Acoust. Soc. Am., vol. 53, no. 3, pp. 742-747, 1973.

[23] T. B. A. Senior, "Low-frequency scattering by a dielectric body," Radio Science, vol. 11, no. 5, pp. 477-482, 1976.

[24] G. C. Lauchle, "Short-wavelength acoustic diffraction by prolate spheroids," J. Acoust. Soc. Am., vol. 58, no. 3, pp. 568-575, 1975.

[25] G. S. Sammelmann, D. H. Trivett, and R. H. Hackman, "High-frequency scattering from rigid prolate spheroids," J. Acoust. Soc. Am., vol. 83, no. 1, pp. 46-54, 1988.

[26] Z. Ye, "Low-frequency acoustic scattering by gas-filled prolate spheroids in liquids," J. Acoust. Soc. Am., vol. 101, no. 4, pp. 1945-1952, 1997.

[27] J. W. S. Rayleigh, The Theory of Sound Volume 2. New York: Dover, 1945.

[28] M. Born and R. Oppenheimer, "Zur Quantentheorie der Moleküle," Ann. d. Phys., vol. 84, pp. 457-484, 1927.

[29] L. D. Landau and E. M. Lifshitz, Quantum Mechanics. Amsterdam: Elsevier, 3rd ed., 2003.

[30] M. Abramowitz and I. A. Stegun, Handbook of Mathematical Functions with Formulas, Graphs, and Mathematical Tables. Dover, 10th ed., 1972.

[31] C. Flammer, spheroidal wave functions. Stanford California: Stanford University Press, 1957.

[32] C. M. Bender and S. A. Orszag, Advanced Mathematical Methods for Scientists and Engineers. New York: Springer-Verlag, Inc., 1999.

[33] H. Anton and C. Rorres, Elementary Linear Algebra. New Jersey: John Wiley \& Sons, Inc., 9th ed., 2005.

[34] S. Y. Slavyanov and W. Lay, Special Functions: A Unified Theory Based on Singularities. Oxford: Oxford University Press, 2000. 
[35] A. Ronveaux, Heun's Differential Equations. Oxford: Oxford University Press, 1995.

[36] W. G. Baber and H. R. Hassé, "The Two Centre Problem in Wave Mechanics," Proc. Camb. Phil. Soc., vol. 31, pp. 564-581, 1935.

[37] R. T. Birge Proc. Nat. Acad. Sci., vol. 14, 1928.

[38] O. W. Richardson Trans. Faraday Soc., vol. 25, 1929.

[39] D. R. Bates, K. Ledsham, and A. L. Stewart, "Wave Functions of the Hydrogen Molecular Ion," Phil. Trans. Roy. Soc. London. Series A, vol. 246, no. 911, pp. 215-240, 1953.

[40] L. D. Landau and E. M. Lifshitz, Mechanics. Amsterdam: Elsevier, 3rd ed., 2010.

[41] W. Pauli, "Über das Wasserstoffspektrum vom Standpunkt der neuen Quantenmechanik," Zeitschrift für Physik, vol. 36, pp. 336-363, 1926.

[42] H. A. Erikson and E. L. Hill, "A Note on the One-Electron States of Diatomic Molecules,” Phys. Rev., vol. 75, no. 1, pp. 29-31, 1949.

[43] J. D. Jackson, Classical Electrodynamics. New York: John Wiley \& Sons, Inc., 2nd ed., 1975.

[44] D. J. Griffiths, Introduction to Electrodynamics. San Francisco: Pearson Education, Inc., 3rd ed., 2008.

[45] J. Lekner, "Invariants of atom beams," J. Phys. B:At. Mol. Opt. Phys., vol. 37, pp. 1725-1736, 2004.

[46] B. E. A. Saleh and M. C. Teich, Fundamentals of Photonics. New York: John Wiley \& Sons, Inc., 1991.

[47] J. Lekner, "Phase and transport velocities in particle and electromagnetic beams," J. Opt. A:Pure Appl. Opt., vol. 4, pp. 491-499, 2002. 
[48] L. J. El-Jaick and B. D. B. Figueiredo, "A limit of the confluent Heun equation and the Schrödinger equation for an inverted potential and for an electric dipole," J. Math. Phys., vol. 50, pp. 123511: 1-22, 2009.

[49] E. T. Whittaker and G. N. Watson, A Course of Modern Analysis. Cambridge: Cambridge University Press, 4th ed., 1963.

[50] J. Lekner, "Polarization of tightly focused laser beams," J. Opt. A:Pure Appl. Opt., vol. 5, pp. 6-14, 2003.

[51] J. Lekner, "Reply to 'Comment on "TM, TE and 'TEM' beam modes: exact solutions and their problems",", J. Opt. A:Pure Appl. Opt., vol. 4, pp. 219-220, 2002.

[52] A. April, "Nonparaxial elegant Laguerre-Gaussian beams," Opt. Lett., vol. 33, no. 12, pp. 1392-1394, 2008.

[53] A. April, "Nonparaxial TM and TE beams in free space," Opt. Lett., vol. 33, no. 14, pp. 1563-1565, 2008.

[54] A. April, "Power carried by a nonparaxial TM beam," Journal of Optical Society America, vol. 27, no. 1, pp. 76-81, 2010.

[55] N. Bokor and N. Davidson, " $4 \pi$ Focusing with single paraboloid mirror," Optics Communications, vol. 281, pp. 5499-5503, 2008.

[56] M. Zeppenfeld, "Solutions to Maxwell's equations using spheroidal coordinates," New Journal of Physics, vol. 11, pp. 073007:1-17, 2009.

[57] D. J. Griffiths, Introduction to Quantum Mechanics. New Jersey: Pearson Education, Inc., 2nd ed., 2005.

[58] C. Flammer, "The Vector Wave Function Solution of the Diffraction of Electromagnetic Waves by Circular Disks and Apertures. I. Oblate Spheroidal Vector Wave Functions," Journal of Applied Physics., vol. 24, no. 9, pp. 1218-1223, 1953. 
[59] C. Flammer, "The Vector Wave Function Solution of the Diffraction of Electromagnetic Waves by Circular Disks and Apertures. II. The Diffraction Problems," Journal of Applied Physics., vol. 24, no. 9, pp. 1224-1231, 1953.

[60] S. M. Barnett and L. Allen, "Orbital angular momentum and nonparaxial light beams," Optics Communications, vol. 110, pp. 670678, 1994.

[61] J. Lekner, "Invariants of electromagnetic beams," J. Opt. A:Pure Appl. Opt., vol. 6, pp. 204-209, 2004.

[62] J. Lekner, "Invariants of three types of generalized Bessel beams," $J$. Opt. A:Pure Appl. Opt., vol. 6, pp. 837-843, 2004.

[63] J. Lekner, "Acoustic beam invariants," Phys. Rev. E, vol. 75, pp. 036610: $1-6,2007$.

[64] G. N. Watson, Theory of Bessel Functions. Cambridge: Cambridge University Press, 2nd ed., 1944.

[65] H. Bateman, "The solution of partial differential equations by means of definite integrals," Proc. London. Math. Soc., vol. 1, pp. 451-458, 1904.

[66] C. J. Tranter, Integral Transforms in Mathematical Physics. London: Methuen, 3rd ed., 1966.

[67] J. W. S. Rayleigh, "On the Passage of Waves Through Fine Slits in Thin Opaque Screens," Proc. Roy. Soc. London. Series A, vol. 89, no. 609, pp. 194-219, 1913.

[68] C. F. Bohren and D. R. Huffman, Absorption and Scattering of Light by Small Particles. New York: John Wiley \& Sons, Inc., 1983.

[69] S. Asano and G. Yamamoto, "Light Scattering by a Spheroidal Particle," Applied Optics, vol. 14, no. 1, pp. 29-49, 1975.

[70] S. Asano, "Light scattering properties of spheroidal particles," Applied Optics, vol. 18, no. 5, pp. 712-723, 1979. 
[71] K. Heun, "Zur Theorie der Riemann'schen Functionen zweiter Ordnung mit vier Verzweigungspunkten," Math. Ann., vol. 33, pp. 161-179, 1889.

[72] R. S. Maier, "The 192 solutions of the Heun equation," Math. Comp., vol. 76, pp. 811-843, 2007.

[73] P. P. Fiziev, "Novel relations and new properties of confluent Heun's functions and their derivatives of arbitrary order," J. Phys. A:Math. Theor., vol. 43, no. 3, pp. 035203:1-9, 2010.

[74] H. Exton, "The interaction $V(r)=-Z e^{2} /(r+\beta)$ and the confluent Heun equation," J. Phys.A: Math. Gen., vol. 24, pp. L329-L330, 1991. 